Prepared for the U.S. Department of Energy

Under Contract DE-AC05-76RL01830

\title{
FY2008 Calibration Systems Final Report
}

\author{
BD Cannon \\ TL Myers \\ BT Broocks
}

January 2009

\section{Pacific Northwest}




\title{
DISCLAIMER
}

This report was prepared as an account of work sponsored by an agency of the United States Government. Neither the United States Government nor any agency thereof, nor Battelle Memorial Institute, nor any of their employees, makes any warranty, express or implied, or assumes any legal liability or responsibility for the accuracy, completeness, or usefulness of any information, apparatus, product, or process disclosed, or represents that its use would not infringe privately owned rights. Reference herein to any specific commercial product, process, or service by trade name, trademark, manufacturer, or otherwise does not necessarily constitute or imply its endorsement, recommendation, or favoring by the United States Government or any agency thereof, or Battelle Memorial Institute. The views and opinions of authors expressed herein do not necessarily state or reflect those of the United States Government or any agency thereof.

\author{
PACIFIC NORTHWEST NATIONAL LABORATORY \\ operated by \\ BATTELLE \\ for the \\ UNITED STATES DEPARTMENT OF ENERGY \\ under Contract DE-AC05-76RL01830 \\ Printed in the United States of America \\ Available to DOE and DOE contractors from the \\ Office of Scientific and Technical Information, \\ P.O. Box 62, Oak Ridge, TN 37831-0062; \\ ph: (865) 576-8401 \\ fax: (865) 576-5728 \\ email: reports@adonis.osti.gov

\begin{abstract}
Available to the public from the National Technical Information Service, U.S. Department of Commerce, 5285 Port Royal Rd., Springfield, VA 22161

ph: (800) 553-6847$$
\text { fax: (703) 605-6900 }
$$

email: orders@ntis.fedworld.gov
\end{abstract} \\ online ordering: http://www.ntis.gov/ordering.htm
}

This document was printed on recycled paper.

$(9 / 2003)$ 
PNNL-18180

\title{
FY2008 Calibration Systems Final Report
}

\author{
BD Cannon \\ TL Myers \\ BT Broocks
}

January 2009

Prepared for the U.S. Department of Energy under Contract DE-AC05-76RL01830

Pacific Northwest National Laboratory

Richland, Washington 99352 



\section{Executive Summary}

The Calibrations project has been exploring alternative technologies for calibration of passive sensors in the infrared (IR) spectral region. In particular, we have investigated using quantum cascade lasers (QCLs) because these devices offer several advantages over conventional blackbodies such as reductions in size and weight while providing a spectral source in the IR with high output power. These devices can provide a rapid, multi-level radiance scheme to fit any nonlinear behavior as well as a spectral calibration that includes the fore-optics, which is currently not available for on-board calibration systems.

In the first two years of the project, we focused on testing the stability and repeatability of cryogenically cooled QCLs because QCLs that operated at room temperature were not available. These preliminary studies demonstrated that we could ramp the current of a cryogenically cooled QCL to provide six radiance levels in less than two minutes. We then tested the short- and long-term repeatability of this multi-level radiometric scheme by continually cycling the current to the cryogenically cooled laser, which was stabilized only with high precision current controllers and closed-loop temperature control. The repeatability over a year was demonstrated to be around $\pm 2.5 \%$ and was limited by a cyclic variation that depended on the cryogenic cooling.

At the end of FY06, we began to test thermoelectrically cooled (TEC) QCLs due to advancements in QCL technology that enabled devices to operate continuous-wave at room temperature. We were able to demonstrate these devices are as robust to power cycling as their cryogenically cooled counterparts. Furthermore, the repeatability over a year was demonstrated to be around $\pm 0.5 \%$ with no evidence of a cyclic variation. Because better long-term performance was achieved with the TEC-QCLs, we discontinued testing the cryogenically cooled devices and focused our efforts on the TEC-QCLs.

The power measurements are primarily limited by temperature fluctuations so that in FY07, we built a custom environmental enclosure to provide freedom from air currents and vibrations in the laboratory. This enclosure enabled us to achieve uncertainties better than $0.1 \%$ for data collected over several days of operation. In FY08, the final year of this project, we continued to improve the measurement set-up and to identify and remove sources of variability. The largest improvement was a reduction in drifts in the power meter offset by stabilizing the temperature for the thermopile head and the QCL mount. We are now able to achieve a standard deviation of approximately $3 \mu \mathrm{W}$ on a $30 \mathrm{~mW}$ scale for periods of up to two weeks. This uncertainty has facilitated stability measurements of $\pm 0.02 \%$ over 16 days of operation.

One variation that appears to be intrinsic to the QCL is a burn-in that has been observed in almost all of the TEC-QCLs tested to date. This burn-in results in an increase in power and currently is the limiting factor in the long-term repeatability measurements. Attempts to reduce the magnitude of the burn-in by thermal annealing prior to operation have been unsuccessful. It is unclear if this effect would be observed in all QCLs or if it depends on the manufacturer as well as the QCL design. The effect, however, can be minimized by running current through the devices prior to use in a calibration system. 




\section{Acronyms and Abbreviations}

$\begin{array}{ll}\text { ADC } & \text { analog-to-digital converter } \\ \text { AIN } & \text { aluminum nitride } \\ \text { CW } & \text { continuous wave } \\ \text { DAC } & \text { digital-to-analog converter } \\ \text { DFB } & \text { distributed feedback } \\ \text { DMM } & \text { digital multimeter } \\ \text { FP } & \text { Fabry-Perot } \\ \text { FPA } & \text { focal plane array } \\ \text { FTIR } & \text { Fourier transform infrared } \\ \text { GPIB } & \text { General Purpose Interface Bus } \\ \text { HHL } & \text { high heat load } \\ \text { HIS } & \text { hyperspectral imaging spectrometers } \\ \text { HR } & \text { high-reflector } \\ \text { HVAC } & \text { heating, ventilation and air conditioning } \\ \text { ILX } & \text { ILX Lightwave } \\ \text { IR } & \text { infrared } \\ \text { JFET } & \text { junction gate field-effect transistor } \\ \text { L-I } & \text { power versus peak current } \\ \text { LWIR } & \text { long-wavelength infrared } \\ \text { MWIR } & \text { mid-wave infrared } \\ \text { NA } & \text { numerical aperture } \\ \text { PNNL } & \text { Pacific Northwest National Laboratory } \\ \text { PRT } & \text { platinum resistance thermometer } \\ \text { QC } & \text { quantum cascade } \\ \text { QC5 } & \text { fifth version of custom current controller } \\ \text { QC6N } & \text { sixth version of custom current controller } \\ \text { QCL } & \text { quantum cascade laser } \\ \text { RMS } & \text { root-mean square } \\ \text { TEC } & \\ \text { thermoelectrically cooled }\end{array}$





\section{Contents}

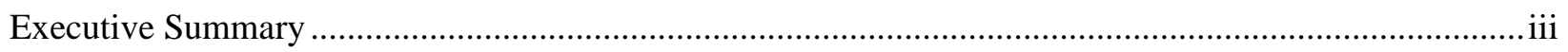

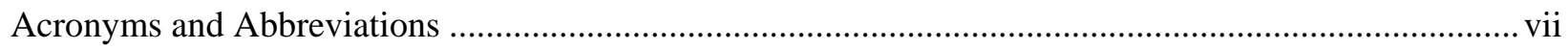

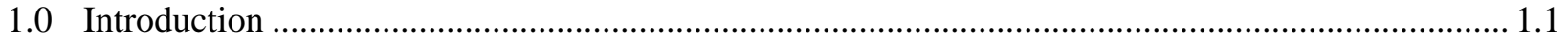

2.0 Stability of Continuous-Wave Thermoelectrically Cooled QCLs ................................................ 2.1

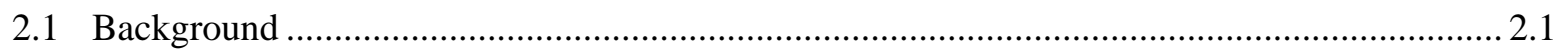

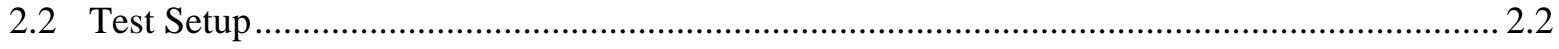

2.3 Identifying and Fixing Sources of Variations in Power Readings .......................................... 2.3

2.3.1 Sources of Changes in QCL Output Power .............................................................. 2.4

2.3.2 Sources of Power Meter Reading Errors ....................................................................... 2.9

2.3.3 Power Meter Readout........................................................................................... 2.14

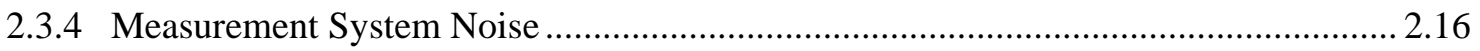

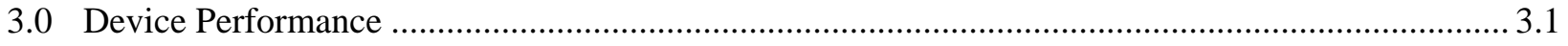

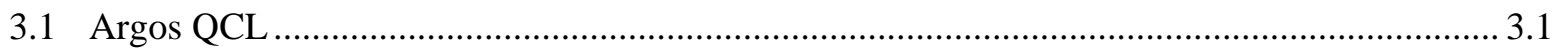

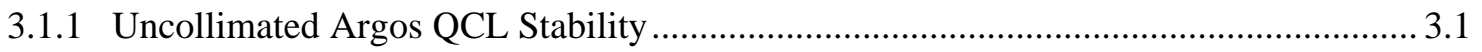

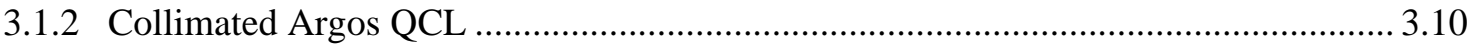

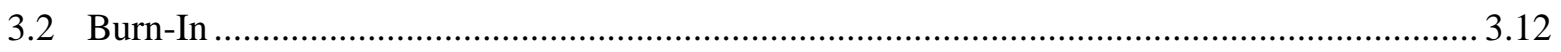

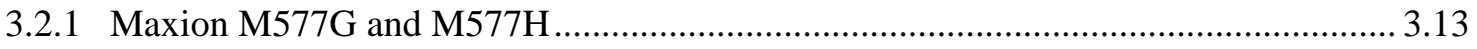

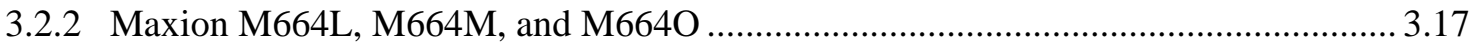

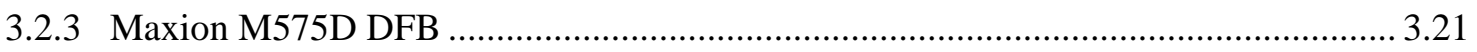

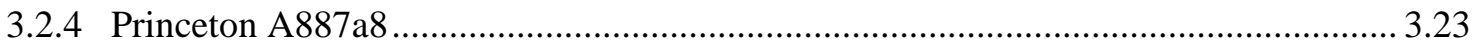

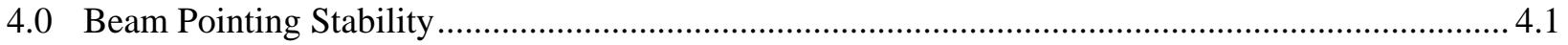

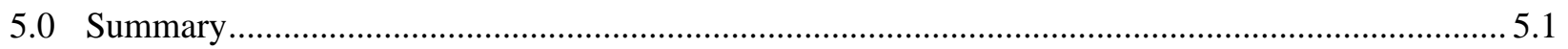

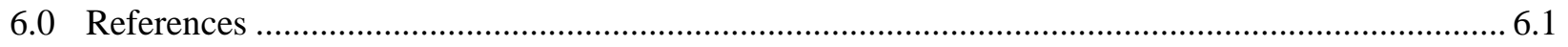

Appendix A Thermopile Amplifier........................................................................................................ A.1 


\section{Figures}

2.1 Simple Environmental Enclosure for Stability Testing.....

2.2 Power Deviations from the Mean at Five Different Current Levels for a QC Laser Using a 40-kHz, 50\% Duty Cycle Current Modulation in the Custom Environmental Chamber ................ 2.2

2.3 Schematic of Filter on Current Monitor Output of QC5 Current Controller.................................. 2.3

2.4 Power Versus Current Plot for the Argos QCL at $20^{\circ} \mathrm{C}$ Using the $40 \mathrm{kHz}$ Modulation ................. 2.6

2.5 Warm-up of QC5 Current Controller with Initial Use................................................................ 2.7

2.6 Changes in QC5 Current Controller Output with Temperature as a Function of Average

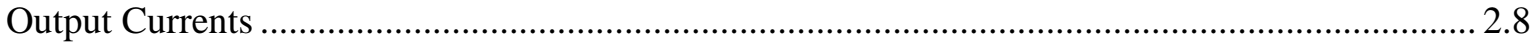

2.7 Variations in Laboratory Temperature from November 7, 2007, to December 9, 2007 ................ 2.9

2.8 Correlation Between Laboratory Temperature and Power Meter Zero Readings ........................ 2.11

2.9 Correlations of Power Meter Head Temperature with Laboratory Temperature and Power Meter Zero Readings .

2.10 Variations in Power Readings over 10 Days at Argos QCL Output Powers of about 6, 12,

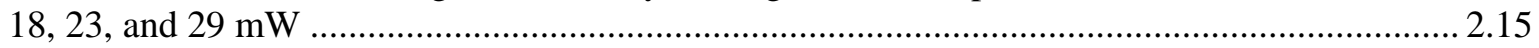

2.11 Zero Differences of 3Sigma ${ }^{\mathrm{TM}}$ Readout and Custom Readout Amplifier ................................... 2.16

2.12 Correspondence of Power Readings with 3Sigma ${ }^{\mathrm{TM}}$ Readout and Custom Readout

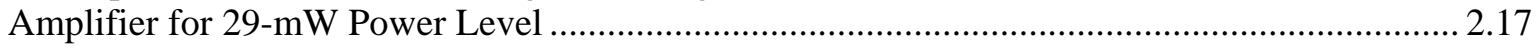

2.13 Variability of Measurement System Readings as a Function of Measurement Duration.............. 2.17

3.1 First Month of Data from the Argos QCL Using the Modulated Excitation .................................. 3.2

3.2 Argos Power Readings from the Initial Ability to Measure QCL Compliance Voltage While Using the Modulated Current Waveform Until the End of 2007....................................... 3.2

3.3 Argos Power Readings for the Second Quarter of FY08 .......................................................... 3.3

3.4 Drift Rates in Power Readings from January 2, 2008, to February 4, 2008, and Predicted Power Reading Drift Rates for the Argos QCL Based on Measured Current Monitor and QCL Voltage Drift Rates....

3.5 Power Readings Using the 3Sigma ${ }^{\mathrm{TM}}$ Readout for the Uncollimated Argos QCL After the Improved Stabilization of Power Meter Head Temperature.

3.6 Magnitudes of the QCL Compliance Voltages for Each Power Level Recorded Simultaneously with the Power Readings in Figure 3.5.

3.7 Model Matching Measured Changes in Powers as a Function of Average Power, $\Delta \mathrm{P}(\mathrm{P})$ to a Small Voltage Offset and the Measured Compliance Voltage Differences ................................... 3.8

3.8 First Power Stability Data on Argos QCL .................................................................................. 3.12

3.9 Burn-in of Power and Measured Changes in Current Readings and Compliance Voltage at the 29-mW Power Level for M577G.

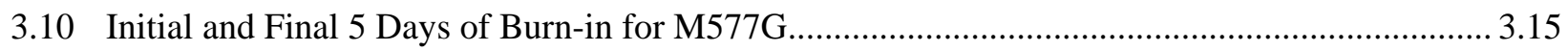

3.11 Burn-in of Power and Measured Changes in Current Readings and Compliance Voltage at the 29-mW Power Level for M577H..

3.12 Initial Power Readings and Readings After the Approximately 250 Hours of the Burn-in Cycle for M577H. 
3.13 M664O Power Readings Using Standard Cycle Following 47.4 Hours of the cw Burn-in Cycle $\quad 3.18$

3.14 Power Stability Measurements of M664M Using the Standard Cycle After the cw Sub-

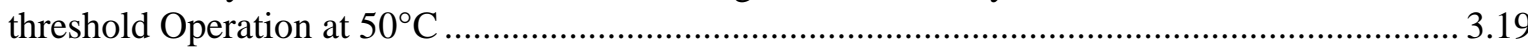

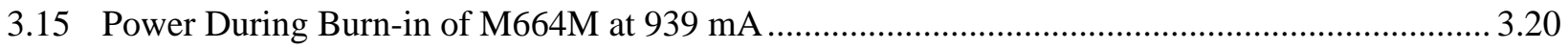

3.16 Power Readings with Standard Cycle for M664 M After 1200-mA Burn in for 5 Days .............. 3.21

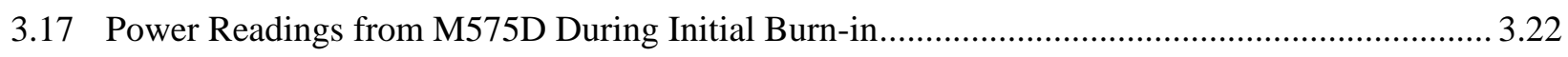

3.18 Power Readings for A887a8 Over 349600 Cycles or 542 Days of Operation ............................. 3.23

3.19 Initial and Final Power Readings for A887a8 Illustrating the Increase in Power that is

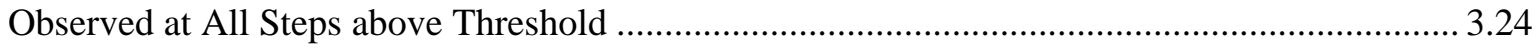

3.20 Power Readings for A887a8 Over 400000 Cycles Illustrating the Sudden Degradation that

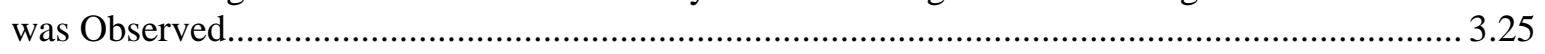

4.1 Beam Profiles Along the Fast Axis for M532A at Different Current Levels ................................. 4.1

4.2 Beam Profiles Along the Slow Axis for M532A at Different Current Levels ............................... 4.2 


\section{Tables}

2.1 Change in the Output Power of the Argos QCL due to a Change in Heat-Sink Temperature ........ 2.4

2.2 Temperature Sensitivity Specifications of Digital-to-Analog Output of NI-6221 Data Acquisition Card.

3.1 Power Stability of the Two Months from 12/27/2007 Until 2/26/2008 of the Uncollimated Argos QCL after Stabilizing the Temperature of the 3Sigma ${ }^{\mathrm{TM}}$ Readout but before Stabilizing the Temperatures of the QC5 Current Controller and Improving the Temperature Stabilization of the Power Meter Head

3.2 Power Stability for the 10 Days Between a) and b) in Figure 3.5 After Improving the Power Meter Head Temperature Stability

3.3 Slopes of Plots of Power Readings Versus QCL Compliance Voltage for the Data Between d) and e) in Figures 3.5 and 3.6.

3.4 Power Stability of the 16 Days from 6/2/2008 Until 6/18/2008 of the Uncollimated Argos QCL After Stabilizing the Temperature of the 3Sigma ${ }^{\mathrm{TM}}$ Readout, Stabilizing the Temperatures of the QC5 Current Controller, and Improving the Temperature Stabilization of the Power Meter Head.

3.5 Power Stability for the Collimated Argos QCL for 14.6 Days.

3.6 Burn-in Slopes from the Initial Data Run for the Argos QCL at Two Slightly Different Temperatures

3.7 Initial Burn-in Slopes for the First Day of Operation...

3.8 Burn-in Slopes for the 5th Day of Operation

3.9 Changes in Power Readings, Current Monitor Readings, and QCL Voltage Readings Between the Fifth Day of Data Collection and the Last Four Days of Data Collection on M577G.

3.10 Changes in Power Readings, Current Monitor Readings, and QCL Voltage Readings Between the Last Day of the Standard Cycle Before Starting to Use the Burn-in Cycle and the Last Four Days of Data Collection on M577H.

3.11 Changes in Power Readings, Current Monitor Readings, and QCL Voltage Readings Between the Last Day of the Standard Cycle Before Starting to Use the cw Burn-in Cylce for 47.4 Hours and the Last Day of Data Collection on M664O.....

3.12 Changes in Power Readings, Current Monitor Readings, and QCL Voltage Readings Between the Last Day of the Standard Cycle Before the 939 mA Burn-in and the First Day of the Standard Cycle Measurements Afterwards

3.13 Changes in Power Readings, Current Monitor Readings, and QCL Voltage Readings Between the Last Day of the Standard Cycle Before the 1200-mA Burn-in and the First Day of the Standard Cycle Measurements Afterwards.

3.14 Long-Term Power Stability for A8887a8 Over 542 Days of Operation ...................................... 3.23

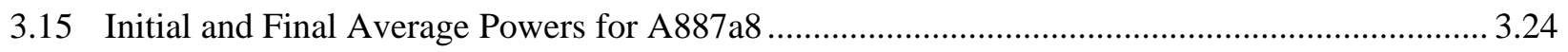

3.16 Power Stability for A887a8 After the Device has been Burned in.............................................. 3.25

3.17 Slope Efficiencies and Threshold Currents for Sections of Data from Figure 3.20 ..................... 3.26 


\subsection{Introduction}

Quantum cascade (QC) lasers are semiconductor devices that may provide a revolutionary technology for calibration of passive instruments in the long-wavelength and mid-wave infrared (LWIR/MWIR) region. One of the major limitations for fielding compact hyperspectral imaging spectrometers (HIS) in the IR is the size, weight, and power of a conventional calibration system based on two internal blackbodies. Furthermore, a two-blackbody system can only provide a linear check for the pixel response because multi-level radiance schemes are time-consuming due to the slow time-constants of the blackbody emitters. Using the QCL, however, the output power can be rapidly swept over the dynamic range of the detectors to provide a nonlinearity correction and to monitor any changes during the lifetime of the instrument. Additionally, the use of emitted light from multiple QCLs can be employed to cover a wide spectral region of interest across the infrared (3.5-17 $\mu \mathrm{m})$. Therefore, this unique calibration method based on QCLs may offer a viable alternative to blackbodies and result in a more compact system that allows more complex calibration strategies by monitoring both spectral and power response focal plane array (FPA) variations.

This work investigates if QCLs can be used as a stable and accurate radiometric and spectral calibration source for the IR wavelengths to help address the current challenges for calibration in the IR. A multi-level radiance scheme has been demonstrated to provide a rapid nonlinearity correction that goes beyond a traditional two-point calibration scheme based on two internal blackbodies. This scheme cycles the power to the QCL in order to minimize the impact of power on the instrument payload. Recent advances in room-temperature operation of QCLs have also enabled us to focus on thermoelectrically cooled (TEC) QCLs, eliminating the need for cryogenic consumables as well as the problems observed with thermal cycling.

Section 2.0 contains a discussion of the stability of thermoelectrically cooled QCLs and the attempts to identify and correct for sources of variation in the power readings, most of which are due to the measurement set-up. Section 3.0 presents the power measurements for multiple thermoelectrically cooled devices and investigates the burn-in that has been observed in almost all of the devices tested to date, as well as our efforts to reduce the burn-in. The long-term repeatability measurements for one device that has been tested for over two years are also included. Section 4.0 shows some of the measurements that illustrate the beam pointing is stable with changes in current. Finally, a summary of the results is presented in Section 5.0 and references are provided in Section 6.0. 



\subsection{Stability of Continuous-Wave Thermoelectrically Cooled QCLs}

\subsection{Background}

As detailed in the FY07 annual report for this project (Myers et al. 2007), we investigated the role of thermopile stability in our measurements of the power stability of QCLs. We established that we could reduce the contribution of the thermopile to the variations in our power measurements to a standard deviation of about $3 \mu \mathrm{W}$ with a $30 \mathrm{~mW}$ full-scale range over a period of about two weeks. We attained this performance in an inexpensive, custom environmental enclosure that provided freedom from air currents and vibrations as well as a stable temperature for the power meter head and the QCL mount. Figure 2.1 shows this double-walled enclosure with clear plastic tubing carrying deionized water between a circulator and the aluminum heat exchanger on the back of the black QCL mount.

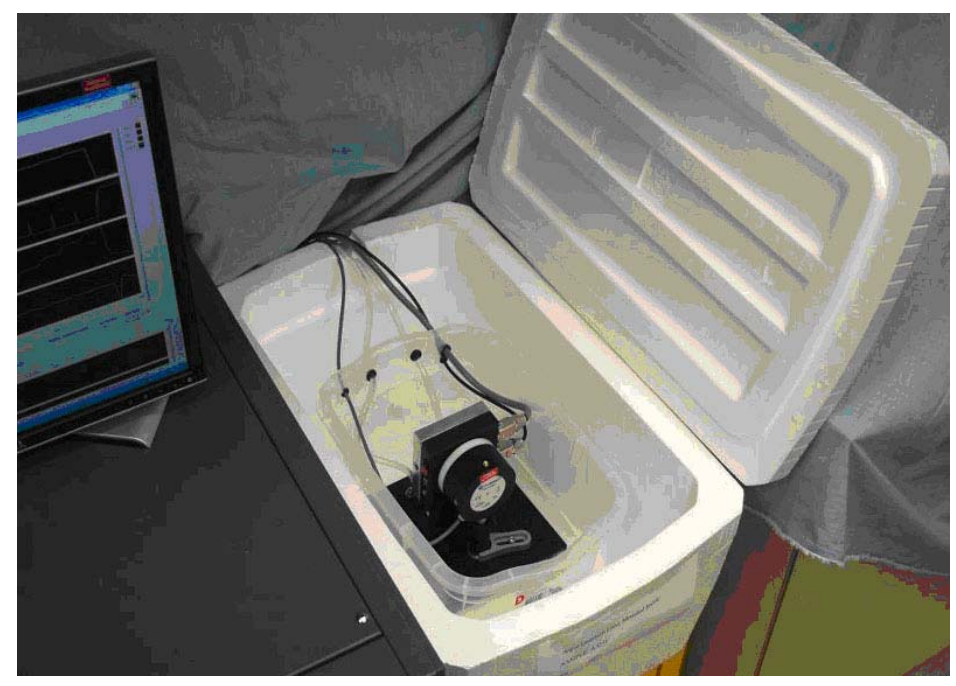

Figure 2.1. Simple Environmental Enclosure for Stability Testing

Initial tests in this enclosure with a QCL operating continuous-wave above threshold at room temperature were disappointing with power variations between 8 and 25 times larger than with this same laser operating below threshold. We hypothesized that the source of these much greater variations was the stochastic nature of the selection of which modes of the QCL reach threshold first upon bringing the current above threshold. Using modulated current to power the QCL both confirmed this hypothesis and reduced the power variations over almost three days to near the values measured with this QCL below threshold. Square-wave modulation of the current at a frequency of $40 \mathrm{kHz}$ with $50 \%$ duty cycle and $100 \%$ amplitude was fed to the "Remote" input to the current controller. As FY07 ended, we had 10 days of data (see Figure 2.2) with this configuration, which showed a longer term drift, which appeared to have stopped, and a significant fraction of the power variations was common to all five tested power levels. Substantial work has been done in FY08 to investigate these two features and determine what is due to the test apparatus and what to the QCL itself. 


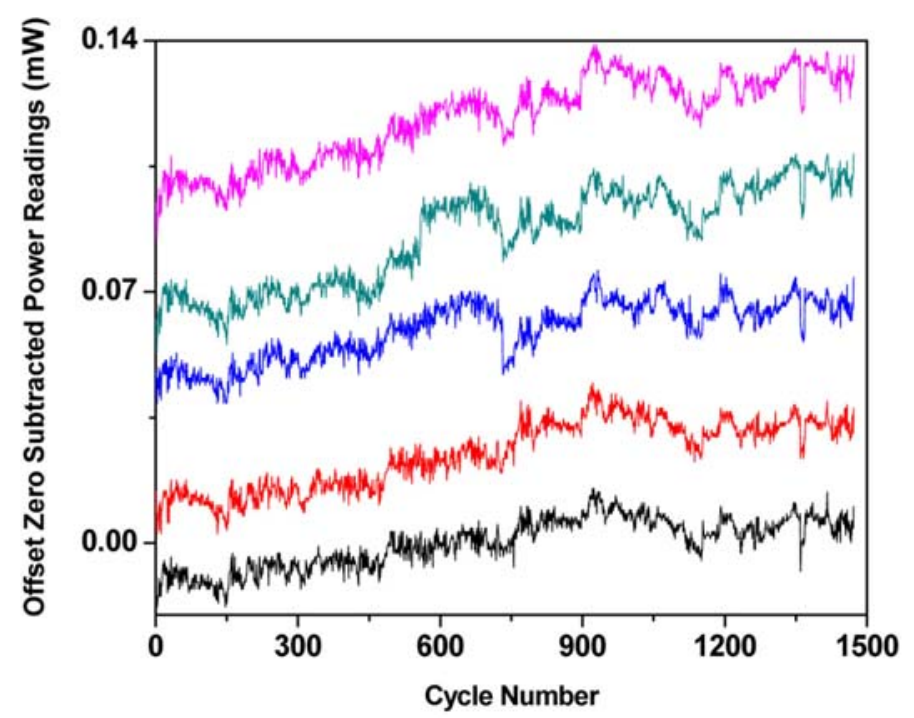

Figure 2.2. Power Deviations from the Mean at Five Different Current Levels for a QC Laser Using a 40-kHz, 50\% Duty Cycle Current Modulation in the Custom Environmental Chamber. The black, red, blue, dark cyan, and magenta traces represent the first through the fifth steps above threshold, respectively.

\subsection{Test Setup}

At the start of FY08, the custom environmental enclosure housed the power meter head (Coherent Model PS19) and a laser mount (ILX Lightwave Model LDM-4409) that had been modified to replace the air-cooled heat exchanger with an aluminum block through which a NesLab chiller circulated deionized water at $23.0^{\circ} \mathrm{C}$. A half-height instrument rack housed the data acquisition and control computer, a temperature controller for the laser mount (ILX Model LDT-5948), a PNNL-built QCL current controller (Model QC5, negative polarity, 1 A maximum current) and its DC power supply, and the power meter readout (Coherent Model 3Sigma ${ }^{\mathrm{TM}}$ ). All items in this rack were powered by a battery backup, uninterruptable power supply that minimized ground loops and noise pickup from the AC power line. A LabVIEW program controlled the ILX Model LDT-5948 via a GPIB interface, collected power readings from the 3 Sigma $^{\mathrm{TM}}$ via an RS-232 serial link, used a 16-bit digital-to-analog converter (DAC) on a multifunction data acquisition card (National Instruments Model NI 6221), and a 16-bit analog-to-digital converter (ADC) on this same card to read the current monitor output from the QC5 current controller. To improve measurement precision and reduce grounding problems, the ADC inputs on the NI-6221 card were connected in the differential configuration. One such grounding problem was that the current monitor output from the QC5 oscillated when connected to the ADC with a frequency of about $2 \mathrm{MHz}$ and a peak-to-peak amplitude of about 2 volts. Figure 2.3 shows the schematic of the filter used between the current monitor output and the ADC input, which both prevents the oscillation and provides low-pass filtering with a corner frequency of $32 \mathrm{~Hz}$. The inductors in the center and shield paths prevent oscillation and the low-pass filtering greatly reduces the $40-\mathrm{kHz}$ modulation and passes the average value of the current monitor output to the ADC. 


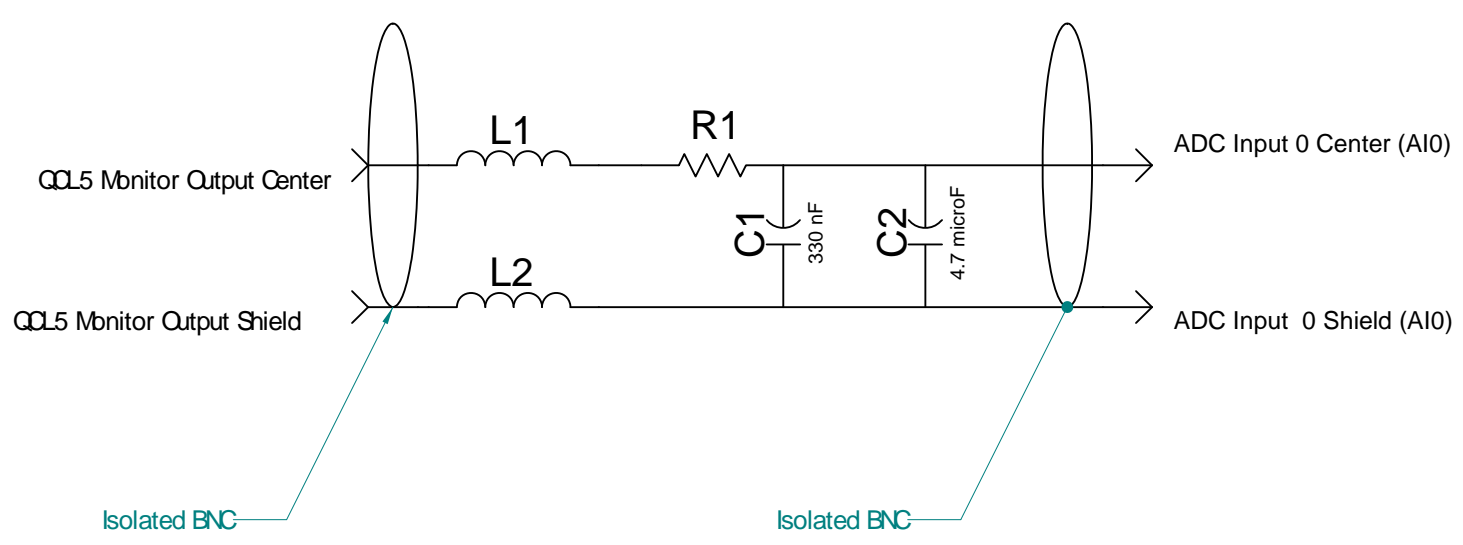

Figure 2.3. Schematic of Filter on Current Monitor Output of QC5 Current Controller. The component values are $\mathrm{R} 1=1.0 \mathrm{k} \Omega, \mathrm{C} 1=330 \mathrm{nF}+1 \mathrm{nF}, \mathrm{C} 2=4.7 \mu \mathrm{F}$ tantalum (signal is negative), and $\mathrm{L} 1=\mathrm{L} 2=100 \mu \mathrm{H}$.

To help distinguish between power reading variations due to the QCL and those due to components of the testing apparatus, we measured the voltage across the QCL in a four-wire configuration. This used a pair of wires between the connector on the laser mount and ADC inputs 2 and 10 for a differential measurement. This four-wire configuration eliminates the errors due to voltage drops in the wires that carry the large current that powers the QCL and the variations due to changes in the resistance of the wires. A modified LabVIEW control program measures the QCL voltage $10 \mu \mathrm{s}$ after the current turns on for each cycle of the $40-\mathrm{kHz}$ modulation and measures the filtered current monitor output $13 \mu \mathrm{s}$ later. These measurements are averaged over 0.5 -second intervals and stored in the data files. This averaging of 20,000 readings provides precision that is better than the 16-bit resolution of the ADC. These delays are synchronized in the hardware of the NI-6221 to the turn-on edge of the modulated signal sent to control the QC5 current controller. The QCL compliance voltage provides a cross-check whether observed power drifts are due to drifts in the current through the QCL versus drifts in the current monitor readings. Using compliance voltage readings and current monitor readings for the seven steps with nonzero currents in our standard measurement cycle, we determined the differential resistance of the QCL. For true changes in QCL current, the ratio of the changes in compliance voltage to the changes in current monitor readings should equal this differential resistance. Changes in the QCL that cause changes in the compliance voltage or changes in the current monitor output or the readings by the ADC of this output that do not reflect changes in the current through the QCL can be identified by examining this ratio.

\subsection{Identifying and Fixing Sources of Variations in Power Readings}

Determining what parameters have the greatest effect on the power readings helps efforts to be focused on problems in the order of their impact. Unfortunately with a complicated system, there can be many mechanisms for environmental variables to impact the stability of the power readings. The mechanisms that impact the variability of power readings can be divided into those that change the actual optical power emitted by the QCL and those that cause the power meter readings to vary for a constant input optical power. In the first category are mechanisms changing the QCL temperature and current as well as changes in the QCL itself, while in the second category are drifts in the power meter head and readout. 


\subsubsection{Sources of Changes in QCL Output Power}

The most direct mechanism affecting the QCL temperature is the temperature of the heat sink to which the QCL is fastened. Table 2.1 shows the dependence of output power with heat-sink temperature for the Argos QCL, which was the most extensively used QCL in the FY08 stability tests. A change in heat-sink temperature causes approximately the same change in optical power at all power levels. This behavior corresponds to changes in heat-sink temperature predominately changing the threshold current and only slightly changing the slope of the optical power versus current curve. There is about an $8 \%$ increase in the magnitude of the change in power with heat-sink temperature between about $5 \mathrm{~mW}$ and $30 \mathrm{~mW}$ for continuous-wave (cw) operation while there is approximately a $20 \%$ increase for a similar power range when operated with modulated current. The largest change in magnitude of this temperature sensitivity is in going from the lowest to the next lowest power level. This behavior in part reflects the curvature of the power versus current curve near threshold for QCLs that is in contrast to the perfectly linear behavior predicted from simple models of laser behavior. With the heat-sink temperature being reproducible to better than $\pm 0.001^{\circ} \mathrm{C}$ as reported in the FY07 annual report, the heat-sink temperature variation should give power variations of less than $\pm 1.4 \mu \mathrm{W}$. This is well below the approximately $3-\mu \mathrm{W}$ standard deviation due to the power meter and so should not be a significant source of power variation.

Table 2.1. Change in the Output Power of the Argos QCL due to a Change in Heat-Sink Temperature. Data are presented for five power levels for operating with both cw currents and with $100 \%$ amplitude modulation of the current at $40 \mathrm{kHz}$ and $50 \%$ duty cycle.

\begin{tabular}{llrrrrc}
\hline cw Operation at $17^{\circ} \mathrm{C}$ & Power Level $(\mathrm{mW})$ & 5.26 & 11.71 & 17.30 & 22.94 & 28.63 \\
& $\Delta$ Power per ${ }^{\circ} \mathrm{C}\left(\mathrm{mW} /{ }^{\circ} \mathrm{C}\right)$ & -1.33 & -1.40 & -1.41 & -1.42 & -1.44 \\
\hline Current Modulated & Power Level $(\mathrm{mW})$ & 5.92 & 11.80 & 17.51 & 23.13 & 28.80 \\
Operation at $20^{\circ} \mathrm{C}$ & $\Delta$ Power per ${ }^{\circ} \mathrm{C}\left(\mathrm{mW} /{ }^{\circ} \mathrm{C}\right)$ & -0.78 & -0.86 & -0.90 & -0.93 & -0.98 \\
\hline
\end{tabular}

However this measured temperature stability does not distinguish between true changes in QCL temperature and changes in the thermistor used to sense the heat-sink temperature, changes in the thermal conduction between the thermistor and the heat sink, and changes in electronic voltage offsets in the temperature controller that controls the heat-sink temperature. Stability of about $\pm 0.0015^{\circ} \mathrm{C}$ with no evidence of long-term drift for more than a year was shown in measurements at the National Bureau of Standards in the 1970s for commercial bead-in-glass thermistors, and in 1992 the National Institute of Standards and Technology reported similar results for glass encapsulated disc thermistors (Wise 1992). Thermistors have been shown to have relative stability at $20^{\circ} \mathrm{C}$ of better than $0.0001^{\circ} \mathrm{C}$ over 44 months (Lawton and Patterson 2001). Additional evidence for the long-term stability of thermistors is the commercial availability of thermistor probes with specified drift rates of $\pm 0.002^{\circ} \mathrm{C}$ per year in the temperature range of $0^{\circ} \mathrm{C}$ and $60^{\circ} \mathrm{C}$ (Fluke 2008) and the use of thermistors in temperature-control systems for telecommunications laser diode sub-assemblies where long-term temperature stability of $\pm 0.001^{\circ} \mathrm{C}$ is required (Lyons and Waterworth 2003). It is thus reasonable to expect that adequate heatsink temperature stability is possible. However, the specified "long-term stability" of the ILX temperature controller of $\pm 0.005^{\circ} \mathrm{C}$ peak-to-peak over 24 hours does not provide any assurance of its stability over weeks or months. This temperature controller could be a significant source of long-term variations, although we do not have any evidence that the QCL power variations we see are due to this controller. 
The threshold and efficiency of a QCL depends primarily on the temperature of the active region of the laser. In steady state, the active-region temperature is higher than the heat-sink temperature by the watts of heat produced in the active region times the thermal resistance to the heat sink. With rapid current modulation, corrections due to the kinetics of the heat transport need to be made to this simple model to fully describe the active-region temperature. Changes in the heat deposited in the active region due to changes in the duty cycle and/or frequency of the current modulation will affect the active-region temperature and hence the optical power. The duty cycle of the current modulation is set by counting "tics" every 50 ns from the 20-MHz clock on the NI-6221. Variations in duty cycle arise from changes in the period of this oscillator over the 25 - $\mu$ s period of the modulation waveform and from differences in the exact level on the fast-rising edges of these tics where the counter makes its transitions. The 50 parts-permillion frequency accuracy of this clock between $0^{\circ} \mathrm{C}$ and $55^{\circ} \mathrm{C}$ and the sub-nanosecond timing jitter of the clock transitions keep the duty cycle variations very small. In addition, these variations are averaged over 40,000 cycles each second, so they are negligible compared to the 100 parts-per-million variations due to the power meter variations. The modulation frequency variation is fixed to the stability of the 20-MHz clock on the NI-6221 and should also be a negligible source of power variations.

Changes in the radiative and conductive heat transfer to the QCL could also affect the active-region temperature, but the temperature control of the custom environmental chamber and the high thermal resistance through still air to the QCL compared to the low thermal resistance between the QCL and the heat sink minimizes such effects. Changing offsets of the current modulation are another possible source of changing the active-region temperature. While ideally the current through the QCL is zero during the "off” part of each cycle, there is an offset in the QC5 that results in a few milliamps of current flowing through the QCL during the "off” part of the cycle. Changes in this offset will change the active-region temperature; however, these effects are very small. The offset current for the QC5 is about $16 \mathrm{~mA}$, which causes a compliance voltage in the Argos QCL of about 0.28 V. The offset current changes with temperature of the QC5 at a rate of $0.053 \mathrm{~mA} /{ }^{\circ} \mathrm{C}$ (see Figure 2.6). As a result, the heat generated in the active region increases by $1 / 2 \times 2 \times 0.28 \mathrm{~V} \times 0.053 \mathrm{~mA} /{ }^{\circ} \mathrm{C}$ or $15 \mu \mathrm{W} /{ }^{\circ} \mathrm{C}$ change in the temperature of the QC5. (Note: The factor of $1 / 2$ comes from the duty cycle and the factor of 2 comes from the change in voltage that accompanies the increase in current.) With a typical thermal resistance for a roomtemperature QCL being about $0.1^{\circ} \mathrm{C} / \mathrm{W}$ (thermal conductance of $10 \mathrm{~W} /{ }^{\circ} \mathrm{C}$ ), the ratio of the change in active-region temperature to the temperature of the QC5 current controller is about $1.5 \times 10^{-6}{ }^{\circ} \mathrm{C} /{ }^{\circ} \mathrm{C}$. From Table 2.1, this corresponds to about a 1-nW change in output power per degree Celsius change in the temperature of the QC5. Finally, changes in the thermal conduction at any of the interfaces between the active region and the heat sink will change the active-region temperature. This is one possible mechanism for changes in the QCL changing the optical power.

Above threshold, the optical power from a QCL increases almost linearly with current until heating effects reduce the efficiency. Figure 2.4 shows a power versus peak current (L-I) plot for the Argos QCL using the modulated current waveform. We approximate the peak current in this waveform as twice the average reading from the filtered current monitor output. The slope of the linear fit is $0.41 \mathrm{~mW}$ per milliamp of peak current with deviations from the fit of about $0.2 \mathrm{~mW}$. The quadratic fit has only a little curvature over the range of the data, but the deviations are about $4 \mu \mathrm{W}$, which is comparable to the variations in the power readings. To match the $3-\mu \mathrm{W}$ standard deviation of the power meter readings, the standard deviation of the current must be $3 \mu \mathrm{W} /(0.41 \mathrm{~mW} / \mathrm{mA})$ or $7 \mu \mathrm{A}$ in the peak currents. For the Argos laser at $20^{\circ} \mathrm{C}$, about $325 \mathrm{~mA}$ of peak current is required for $30 \mathrm{~mW}$ of output, so this requires a 
long-term current stability of about $20 \mathrm{ppm}$. A 7-ppm current stability is required to achieve a 1- $\mu \mathrm{W}$ standard deviation in the power due to current variations.

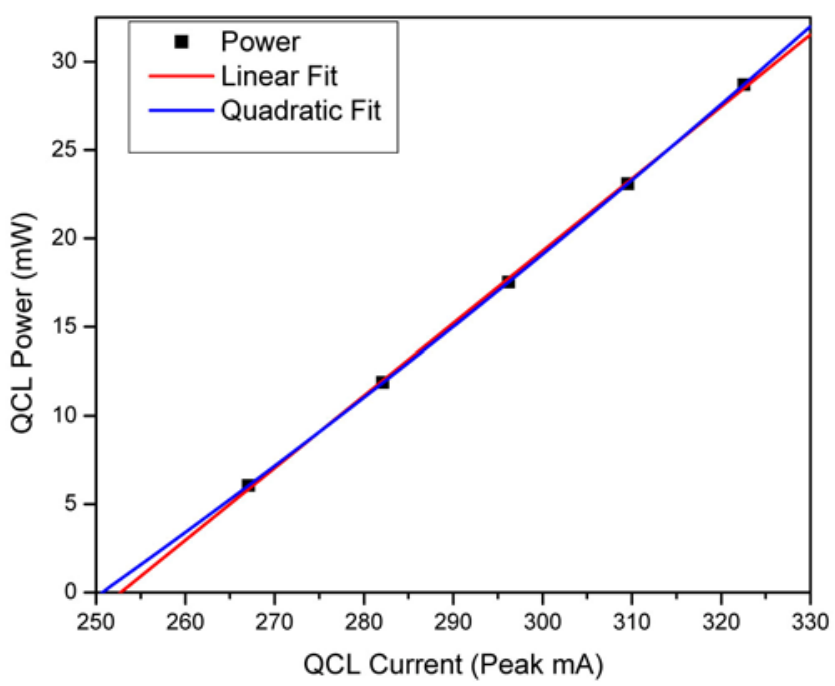

Figure 2.4. Power Versus Current Plot for the Argos QCL at $20^{\circ} \mathrm{C}$ Using the $40 \mathrm{kHz}$ Modulation. While the increase in power with current is nearly linear above threshold, a quadratic polynomial gives a significantly better fit with the magnitude of the residuals of less than $0.005 \mathrm{~mW}$ rather than ranging from $-0.21 \mathrm{~mW}$ to $+0.21 \mathrm{~mW}$ for the linear fit.

The current through the QCL is controlled by the QC5 current controller to a value set by the voltage applied to the "Remote" input by a DAC on the NI-6221 under control of the data acquisition and control program. The two most obvious mechanisms to change the QCL current are (1) changes in the gain and/or offset of the QC5 and (2) changes in gain and/or offset of the DAC feeding the "Remote" input. These gains and offsets change with temperature, either directly as the laboratory temperature changes or indirectly as the components in the QC5 change temperature due to changes in operating conditions such as current levels. We tested the temperature sensitivity of the QC5 current controller by placing it alone in a standard commercial environmental chamber and having it drive an approximately $2 \mathrm{ohm}$ load in series with a 61/2-digit digital multi-meter (Agilent Model 34401A) that measured the average current. All the rest of the apparatus for this test was outside the environmental chamber. After the environmental chamber and the QC5 had equilibrated to the new temperature setting for more than 30 minutes, the data acquisition system stepped the "Remote" input to the QC5 through peak voltages of 0, 2, 3, 4, 5, and 6 volts and then back to zero volts with the current $100 \%$ amplitude modulated at $40 \mathrm{kHz}$ with a $50 \%$ duty cycle with 20 seconds spend for each peak voltage. The current readings from the Agilent 34401A near the end of each 20-second step were manually entered into a spreadsheet. This cycle was repeated 10 times before the temperature setting for the environmental chamber was changed. Data were collected for temperatures of $15^{\circ} \mathrm{C}, 25^{\circ} \mathrm{C}, 35^{\circ} \mathrm{C}, 45^{\circ} \mathrm{C}$, and $55^{\circ} \mathrm{C}$. Figure 2.5 shows data taken at $25^{\circ} \mathrm{C}$ for the $2 \mathrm{~V}$ and $4 \mathrm{~V}$ peak inputs that approximately correspond to $200 \mathrm{~mA}$ and $400 \mathrm{~mA}$ peak currents. The changes in average current between the first point and the last point correspond to power changes for the Argos QCL of about $0.12 \mathrm{~mW}$ and $0.06 \mathrm{~mW}$. These changes in average current are believed to be a result of parts in the QC5 changing temperature as current is delivered by the QC5 to the load. Between each successive point on these curves, the QC5 output has cycled through all the current output steps. It is possible that 
there may be a contribution to these changes with cycle number from current heating the Agilent 34401A input circuit.

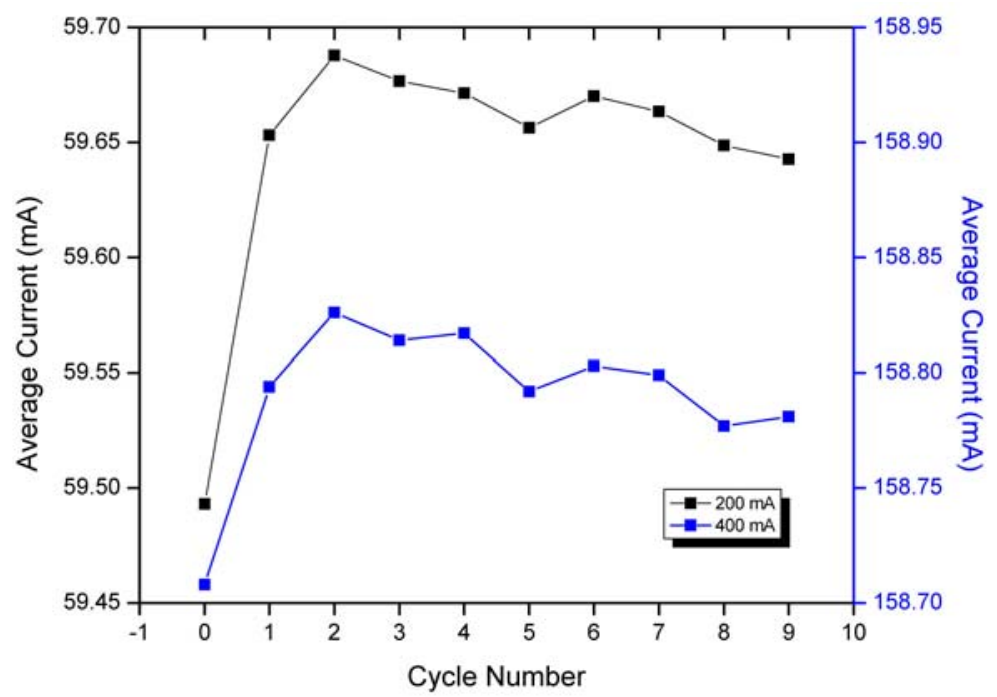

Figure 2.5. Warm-up of QC5 Current Controller with Initial Use

Figure 2.6 shows how the QC5 average output changes with temperature over a range of average output currents, with the error bars coming from the error estimates for the slopes from the linear leastsquares fits to the data for temperatures between $15^{\circ} \mathrm{C}$ and $45^{\circ} \mathrm{C}$. The data from the first two cycles are not included in these fits because they appear to be most strongly influenced by the QC5 warming up. Fitting only the data from the last scan gives values for the temperature dependence of the output that are within $1 \%$ of the results in Figure 2.6. As indicated by the small relative error estimates from the fits, the data are well fit with a linear model for these lower temperatures. All the data at $55^{\circ} \mathrm{C}$ are well below these linear fits. The slope at about $+16 \mathrm{~mA}$ average current corresponds to $0 \mathrm{~V}$ applied to the "Remote" input and so probably represents the combined temperature variations of the offsets in the QC5. Except for the point at an average current of $-107 \mathrm{~mA}$, the points below $0 \mathrm{~mA}$ show a quite linear dependence on average output current. We do not have an explanation for why that one point is low. The effect of a change in the QC5 temperature during the "on" part of the modulation cycle is about $2 \times 0.04 \mathrm{~mA} /{ }^{\circ} \mathrm{C} \times 0.41 \mathrm{~mW} / \mathrm{mA}$ or $30 \mu \mathrm{W} /{ }^{\circ} \mathrm{C}$, where the value of $0.04 \mathrm{~mA} /{ }^{\circ} \mathrm{C}$ comes from Figure 2.6 , the factor of 2 comes from converting from average current to peak current, and the $0.41 \mathrm{~mW} / \mathrm{mA}$ is the slope of the linear fit in Figure 2.4.

The accuracy of the current monitor readings has been checked against these currents measured with the Agilent 34401A as well as similar measurements with the QC5 not in the commercial environmental chamber. The current monitor readings are within $\pm 1 \%$ of those from the Agilent $34401 \mathrm{~A}$ with an offset that is less than $1 \mathrm{~mA}$. The exact correspondence depends on the degree to which the QC5 and the Agilent 34401A are warmed up, the current range measured, and perhaps the compliance voltage seen by the QC5. 


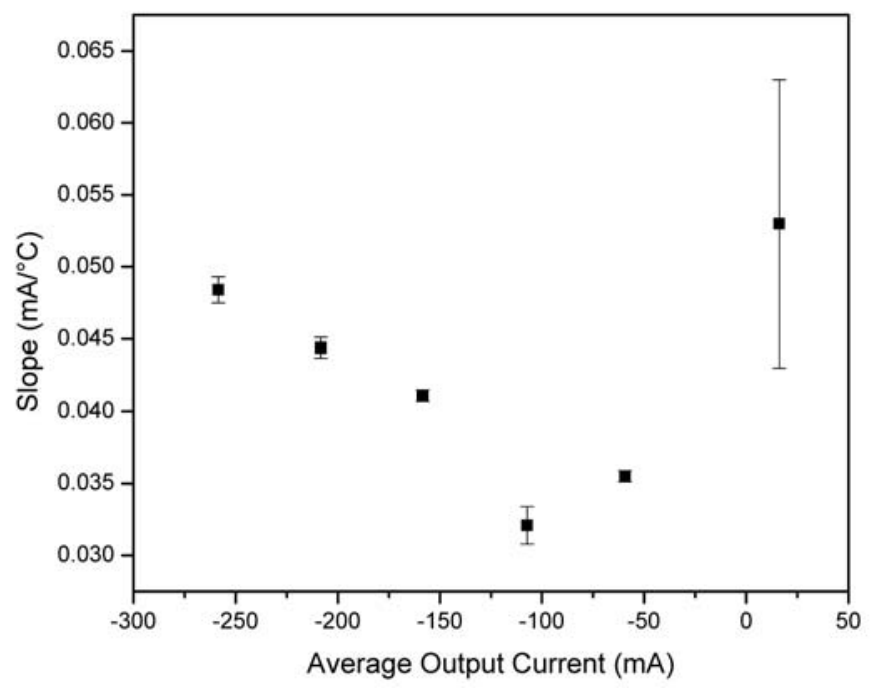

Figure 2.6. Changes in QC5 Current Controller Output with Temperature as a Function of Average Output Currents. The error bars are from the uncertainty in the linear fits of average current versus temperature. The offset from nominally "zero" current has very large error bars due to the significant curvature in the current versus temperature plot.

The voltage output from the National Instruments Model NI-6221 data acquisition card has three parameters that characterize its temperature dependence as specified by the manufacturer, which are given in Table 2.2. Assuming the worst case, that all these temperature sensitivities have the same sign and are the specified maximum values, then for about $30 \mathrm{~mW}$ from the Argos QCL, which requires about a $4 \mathrm{~V}$ output from the NI-6221, these temperature sensitivities would result in power sensitivities for the Argos QCL of $110 \mu \mathrm{V} /{ }^{\circ} \mathrm{C} \times 100 \mathrm{~mA} / \mathrm{V} \times 0.41 \mathrm{~mW} / \mathrm{mA}$ or $4.5 \mu \mathrm{W} /{ }^{\circ} \mathrm{C}$.

Table 2.2. Temperature Sensitivity Specifications of Digital-to-Analog Output of NI-6221 Data Acquisition Card

\begin{tabular}{lcc}
\hline \multicolumn{1}{c}{ Parameter } & Temperature Coefficient & Voltage Change from $\mathbf{4 ~ V}$ \\
\hline Gain & $10 \mathrm{ppm}$ of value $/{ }^{\circ} \mathrm{C}$ & $40 \mu \mathrm{V} /{ }^{\circ} \mathrm{C}$ \\
Voltage Reference & $5 \mathrm{ppm}$ of value $/{ }^{\circ} \mathrm{C}$ & $20 \mu \mathrm{V} /{ }^{\circ} \mathrm{C}$ \\
Offset Tempo & $5 \mathrm{ppm}$ of range $/{ }^{\circ} \mathrm{C}$ & $50 \mu \mathrm{V} /{ }^{\circ} \mathrm{C}$ \\
Total & & $110 \mu \mathrm{V} /{ }^{\circ} \mathrm{C}$ \\
\hline
\end{tabular}

Figure 2.7 shows the laboratory temperature variations from November 7, 2007, to December 9 , 2007, that with peak-to-peak variations of $2.6^{\circ} \mathrm{C}$ and a standard deviation of $0.44^{\circ} \mathrm{C}$ shows larger than average temperature fluctuations. Assuming that these changes in laboratory temperature are tracked by the changes in the NI-6221 card temperature, the contribution to the standard deviation in the Argos QCL power near $30 \mathrm{~mW}$ would be $0.44^{\circ} \mathrm{C} \times 4.5 \mu \mathrm{W} /{ }^{\circ} \mathrm{C}$ or $2.0 \mu \mathrm{W}$. In contrast, between July 22, 2008, and September 2, 2008, the standard deviation of the laboratory temperature was $0.27^{\circ} \mathrm{C}$, which would have contributed 1.2 $\mu \mathrm{W}$ to the Argos QCL power variations due to changes in the NI-6221 temperature. 


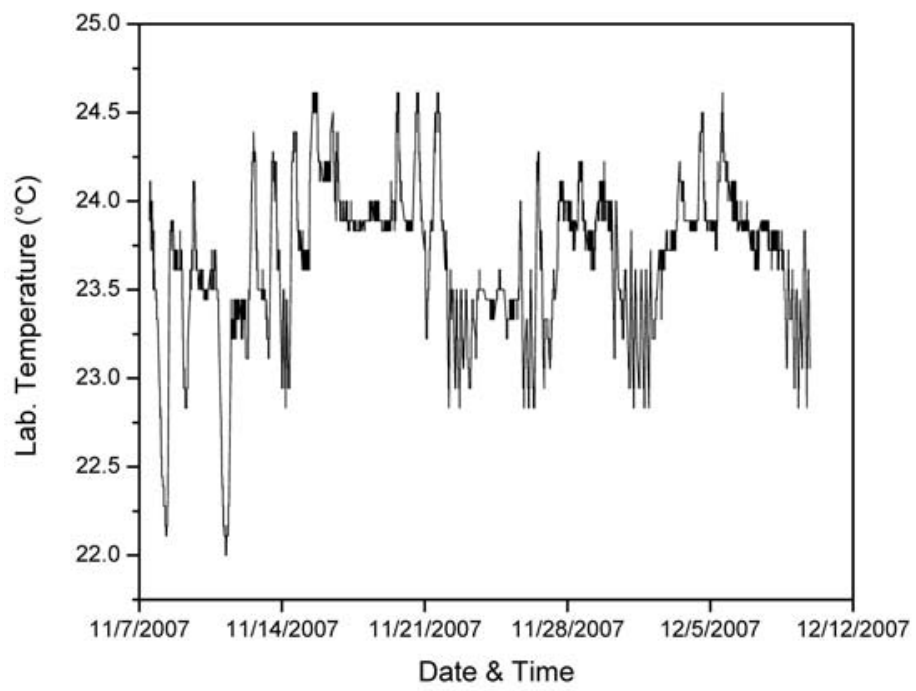

Figure 2.7. Variations in Laboratory Temperature from November 7, 2007, to December 9, 2007. The peak-to-peak variation is $2.6^{\circ} \mathrm{C}$ while the standard deviation is $0.44^{\circ} \mathrm{C}$.

Another source of changes in the QCL power that was found was the potentiometers on the front of the QC5 current controller. These potentiometers do not have knobs or dials on their shafts and have no way to lock them in a fixed position. At the end of November 2007, we discovered that a $0.3-\mathrm{mW}$ increase in all power levels that started in mid-November was due to having accidentally touched the course current adjustment potentiometer on the QC5. To prevent repeating this accidental adjustment, these potentiometers have been removed from the circuit with a soldering iron. Now the only way to adjust the current is by changing the voltage applied to the "Remote" input of the QC5.

\subsubsection{Sources of Power Meter Reading Errors}

The power meter consists of a head, which produces a small voltage that is proportional to the incident optical power, and a separate readout box that amplifies, scales, digitizes, displays, and transmits the readings to the data acquisition computer. Both constituents are potential sources of power meter reading errors.

\subsubsection{Power Meter Head}

The power meter head uses the absorbed optical power to preferentially heat one side of a thermopile that produces a small voltage proportional to the temperature difference between its two sides. Changes in the absorption of the black sensitive surface will change the responsitivity of the power meter head and so give changes in the power readings. This is a significant problem with power meter heads used for small-diameter visible and ultraviolet laser beams that can easily cause visible bleaching of the black sensitive area. For this work, the power meter head was only exposed to the strongly diverging infrared beam directly from quantum cascade lasers in late FY07 and all of FY08, and in the summer of FY07 to a few milliwatts of heat from a small electrically heated resistor used in measuring the noise of the power meter. In addition, the power meter head resided in the custom enclosure for essentially all of FY08 so it 
was well protected from dust and contamination. Unfortunately, with the quoted accuracy of the calibration of this meter by the manufacturer of $\pm 3 \%$, having this meter re-calibrated will not detect changes in the surface absorption that would be significant for this work.

\subsubsection{Zero Readings}

The "Zero" readings of the power meter are an offset because they are the readings with the QCL not lasing and are neither zero nor constant. To correct for drifts in the "zero" readings, we record a zero reading with the QCL held just below threshold at the beginning of each cycle of measurements. As discussed in the FY07 Calibrations annual report (Myers et al. 2007), this significantly reduces the variations in the power readings; but as was also discussed in that report, when the zero is changing rapidly, this procedure does not fully correct for drifts in the zero readings. In November 2007 following changes in the test setup and data acquisition program to (1) improve the precision and accuracy of the current monitor measurements, (2) allow measurement of the QCL compliance voltage when using the modulated current drive, and (3) moving the setup back and forth between different laboratories to measure the temperature sensitivity of the QC5 current controller, we saw increased speed and amplitude of the variations in the power meter zero readings. We suspected that this increased variability in these zero readings was connected to increased variability in the zero subtracted power measurements of the Argos QCL. After a futile effort to connect the increased zero variability to the above changes, we compared measurements of the laboratory temperature recorded every 4.5 minutes, with the power meter zero readings as shown in Figure 2.8(a). Because increases in laboratory temperature correspond to decreases in the power meter zero readings, these zero readings have been multiplied by -1 to highlight the correlation. Additional data from the laboratory in FY08 shows that laboratory-temperature variability increases when the building heating system is operating and is reduced when the building switches over to air conditioning. This appears to be the reason for the increased power meter zero and QCL power reading variability that we observed in November 2007. Because the power meter head was in the custom environmental chamber and the readout was in the instrument rack with a fan blowing laboratory air onto it, we first improved the temperature stability of the readout. The resulting lack of significant improvement established that any temperature sensitivity of the readout was being swamped by some other effect, which turned out to be the temperature sensitivity of the power meter head.

In the last half of February 2008, we inserted a thermocouple that was connected to a data logger into an unused mounting hole in the power meter head and the data logger recorded the head temperature about 4 minutes after the QCL was stepped through its power levels for each cycle. Figure 2.9 shows the strong correlation between the head temperature changes and both the laboratory temperature changes and the changes in power meter zero readings. Plotting the power meter head temperature versus the laboratory temperature from Figure 2.9(a) shows there is some scatter and the slope of least squares line fit to this data is $0.22 \pm 0.01$. (Note: This and other experimental error estimates correspond to twice the standard deviation.) Similar data from February 15-17 and February 18-19 both gave least squares slopes of $0.26 \pm 0.02$. Concerned that the water from the circulating bath was changing temperature in the plastic tubing between the bath and the back plate of the QCL mount, on February 26, 2008, we switched to using an external temperature sensor that was in the flow of water and located within the Styrofoam cooler of the custom environmental chamber as the temperature control point. Laboratory and head temperature data from February 26-28 gave a least squares slope of $0.183 \pm 0.016$, which seemed to indicate that there was some effect but that a stronger mechanism coupling the laboratory temperature to 

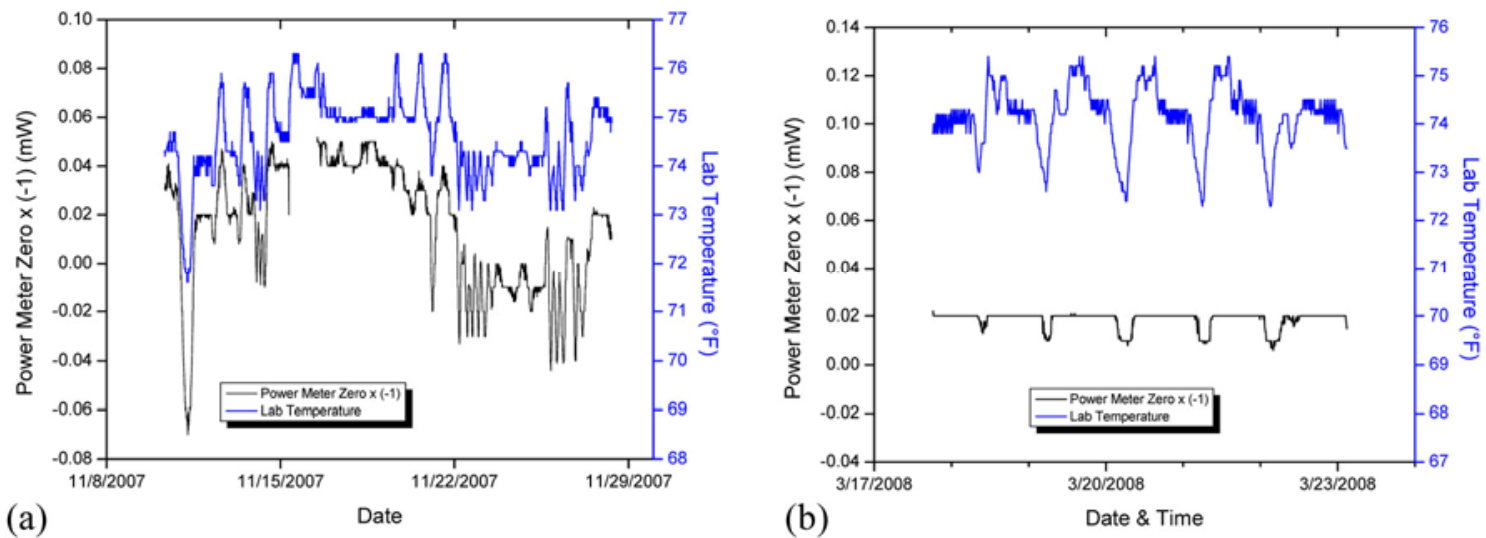

Figure 2.8. Correlation Between Laboratory Temperature (in blue) and Power Meter Zero Readings (in black). (a) Before changes to improve the temperature stabilization of the power meter head. (b) After changes to improve the temperature stabilization of the power meter head. The vertical scales for (a) and (b) are the same showing that the stability of the power meter zero readings has improved about a factor of 10 for about the same range of laboratory temperature variations. The power meter zero readings have been multiplied by -1 to more clearly show the correlation with the laboratory temperature.
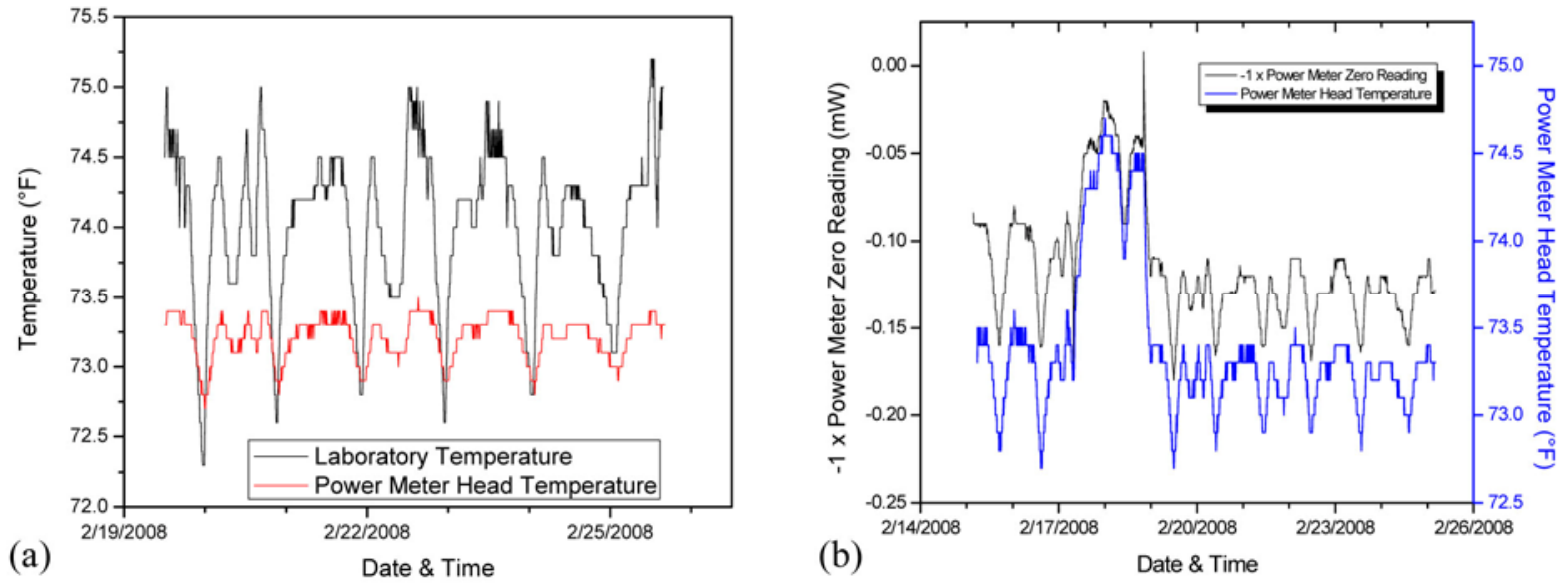

Figure 2.9. Correlations of Power Meter Head Temperature with (a) Laboratory Temperature and (b) Power Meter Zero Readings. The changes in the power meter head temperature are about $1 / 4$ of the changes in the laboratory temperature. The power meter zero readings in (b) have been multiplied by -1 to highlight the correlation of the changes in the power meter zero readings with the head temperature. Included in (b) is the period from the evening of February 17, 2008, until the morning of February 19, 2008, when the temperature of the water circulating through the back plate of the QCL mount was increased in temperature to $23.0^{\circ} \mathrm{C}$ from the value of $22.0^{\circ} \mathrm{C}$ that applies for the rest of the plotted data.

the head temperature was also present. Figure 2.9(b) shows that increasing the temperature of the water circulating through the back plate of the QCL mount from $22.0^{\circ} \mathrm{C}$ to $23.0^{\circ} \mathrm{C}$ for $1 \frac{1}{2}$ days starting the evening of February 17, 2008, increased the head temperature by $0.64 \pm 0.04^{\circ} \mathrm{C}$, based on linear fits to the before, during, and after data sets of laboratory and head temperatures. This is additional evidence that 
the power meter head has substantial thermal contact with something other than the circulating water. This thermal contact suggests a mechanism for the correlation of zero readings with laboratory temperature changes and water temperature: both cause changes in the head temperature, which change the zero readings. Power meter zero readings change with head temperature changes due to laboratory temperature changes at a rate of $-0.170 \pm 0.016 \mathrm{~mW}$ per ${ }^{\circ} \mathrm{C}$ change in the head temperature based on fitting the "before," "during," and "after" sections of data in Figure 2.9(b) and a set of similar data from February 26-28, 2008. Analysis of the data shown in Figure 2.9(b) also gives the rate of zero change with head temperature due to changing the water temperature to be $-0.12 \pm 0.06 \mathrm{~mW} /{ }^{\circ} \mathrm{C}$, which agrees within the estimated error with the previous rate.

To provide insight into how to improve the stability of the zero readings, we developed a simple model of the thermal couplings to the power meter head. The measured temperatures and the changes in the head temperature with changes in water and laboratory temperature are not consistent with only thermal paths connecting the head to the circulating water and to the laboratory air temperature. The simplest model that we found that is consistent with this temperature data assumes electrical power is dissipated in the power head and thermal contact with the cold plate of the QCL mount, which is maintained at $20^{\circ} \mathrm{C}$. Using the circulating water temperature of $22^{\circ} \mathrm{C}$, the average laboratory air temperature of $23.3^{\circ} \mathrm{C}$, the average head temperature of $22.9^{\circ} \mathrm{C}$, and the sensitivities of the head temperature to water and laboratory air temperature, conservation of power at steady state lets us write three linear equations with four unknowns, values for the thermal conduction for the three thermal paths and the electrical power dissipated in the power meter head. We can solve this model by independently calculating the thermal conduction between the cold plate and the power meter head.

One major mechanism for thermal contact between the head and the cold plate is conduction through the air between the 2-in. $\times 2$-in. cold plate that is positioned about $1 / 4$-in. from the front of the power meter head. With the thermal conductivity of air of $0.26 \mathrm{~mW} /\left(\mathrm{cm}^{\circ} \mathrm{C}\right)$, the thermal conduction between the head and the cold plate is about $10 \mathrm{~mW} /{ }^{\circ} \mathrm{C}$ in the absence of convection. The other obvious mechanism for this thermal contact is radiative transfer. Radiative heat transfer between a pair of closely spaced parallel "gray body" plates with emissivities of $\varepsilon_{H}$ and $\varepsilon_{L}$, temperatures $T_{L}+\Delta T$ and $T_{L}$, and area $A$ is given by

$$
4 A \sigma_{S B} T_{L}^{3}\left(\frac{\varepsilon_{H} \varepsilon_{L}}{\varepsilon_{H}+\varepsilon_{L}-\varepsilon_{H} \cdot \varepsilon_{L}}\right) \cdot \Delta T
$$

where $\sigma_{S B}$ is the Stefan-Boltzman constant. Because the emissivity of the gold-coated cold plate is low, estimated to be about $0.05-0.1$, the thermal conduction due to radiation between QCL mount's cold plate and the power meter head is estimated to be $0.7-1.4 \mathrm{~mW} /{ }^{\circ} \mathrm{C}$. Thus, the thermal conduction between the head and the cold plate is about $11 \mathrm{~mW} /{ }^{\circ} \mathrm{C}$, unless the emissivity of the gold coating is much larger than estimated.

With this thermal conduction value, we solve the model to predict that $70 \mathrm{~mW}$ of electrical power are dissipated in the head and that the thermal conduction to the water is $50 \mathrm{~mW} /{ }^{\circ} \mathrm{C}$ and to the laboratory air is $17 \mathrm{~mW} /{ }^{\circ} \mathrm{C}$. The power result is reasonable for a pre-amplifier that is probably in the power meter head. The value for the conduction between the head and the laboratory air is surprising because it is larger than the value for conduction to the cold plate that is in close proximity. However, radiative coupling from the surfaces of the power meter head that are not facing the QCL mount may be a reasonable mechanism to explain this level of thermal conduction. Black body emission and absorption can provide $115 \mathrm{~mW} /{ }^{\circ} \mathrm{C}$ of 
thermal conduction if the emissivity of the power meter head is close to unity and the walls of the plastic tub and Styrofoam cooler have enough transmission in the long wavelength infrared. This is suggestive that the model's prediction of the thermal conduction to the laboratory temperature is not unreasonable, but is much larger than expected. Another mechanism would be development of convection currents in the air spaces within the custom environmental chamber, but the heat transfer by this mechanism is not easy to estimate.

This model suggests three approaches to stabilizing the power meter zero readings: (1) increase thermal conduction between the head and the circulating temperature-controlled water, (2) improve the temperature stability of the water where there is maximum thermal conduction to the head, and (3) reduce the radiative coupling of the head to the environment. The first approach was implemented by inserting a length of copper tubing into the water circulation path that was connected by a pair of copper taps soldered onto the tubing and fastened onto the two metric mounting holes on the side of the power meter head with M6 brass screws. The second approach was implemented by controlling the water temperature based on an external temperature sensor that was immersed in the flow inside the Styrofoam cooler and directly connected to the copper tubing described in the previous sentence. Aluminum foil wrapped around the surfaces of the power meter not facing the QCL mount was added to reduce the radiative coupling. Figure 2.8(b) shows the factor of 10 improvement in the power meter zero variations following implementation of these changes. With this improvement in the zero stability, it was very disappointing that the variation in the zero subtracted QCL power measurements corresponding to the 6 days of data in Figure 2.9(a) was between $4.8 \mu \mathrm{W}$ to $6.9 \mu \mathrm{W}$ while for $5 \frac{1}{2} 2$ days of data collected after these improvements, the power measurement variations were $5.2 \mu \mathrm{W}$ to $11 \mu \mathrm{W}$. Much of the variation in this later data was clearly correlated with changes in laboratory temperature, which was more variable for the later data set than for the former. This "disappointing” result still corresponds to $0.06 \%$ stability (twice the relative standard deviation) for the 29-mW step in the cycle. Shortly after this later data collection was finished and following re-booting the computer and cycling the power for the QCL mount's temperature controller, we collected 10 days of data with variations in power readings between $4.1 \mu \mathrm{W}$ and $4.9 \mu \mathrm{W}$ and demonstrated a stability of $0.034 \%$ for the $29-\mathrm{mW}$ step in the cycle.

The power meter zero reading also changes with the amount of heat dissipated by the QCL. However, it is remarkable how small a fraction of that heat is registered by the power meter. When taking the current through the Argos QCL from "zero" to just below threshold, the heat dissipated in the QCL increases by about $0.9 \mathrm{~W}$, but the power meter reading only increases by about $0.02 \mathrm{~mW}$ ! At the maximum current used, which gave $29 \mathrm{~mW}$ of optical output, the heat dissipated increases to about $1.2 \mathrm{~W}$, which should increase the power reading by $0.007 \mathrm{~mW}$. For a QCL with a higher threshold, Maxion M664O, the heat dissipated just below threshold is almost $2.75 \mathrm{~W}$ and the power meter reading increase is about $0.08 \mathrm{~mW}$. For $29 \mathrm{~mW}$ of optical output from this QCL, the heat dissipation is $3.4 \mathrm{~W}$, which should increase the power reading by $0.02 \mathrm{~mW}$. These increases to the power meter readings due to increases in heat from the QCL increases above the values just below threshold where the power meter zero readings are recorded are not subtracted from the power readings, but they are a small systematic error.

\subsubsection{Change in Power Meter Head Calibration}

There are three obvious mechanisms by which the power meter head calibration can change. One is changes in the absorption of the black coating on the sensitive surface, which has been discussed above. 
The other two are changes in the thermopile itself and changes in the gain of the pre-amplifier in the head. After the improvements in the thermal stabilization, the head's temperature is stable to better than $\pm 0.02^{\circ} \mathrm{C}$. Because the head has a calibration uncertainty of $\pm 3 \%$ and is intended for use in a laboratory over a temperature range from about $15^{\circ} \mathrm{C}$ to $30^{\circ} \mathrm{C}$, the combined response of the thermopile and the preamplifier should change much less than $\pm 3 \%$ over this temperature range. Over a temperature range of $\pm 0.02^{\circ} \mathrm{C}$, this combined response should be constant to \pm 40 parts-per-million assuming a linear variation with temperature. This value is well within the expected stability of electrical components such as resistors for this level of temperature stabilization.

\subsubsection{Power Meter Readout}

\subsubsection{Zero and Gain Variation}

Zero drifts and gain changes in the 3Sigma ${ }^{\mathrm{TM}}$ digital readout for the power meter head are the obvious potential sources of variations due to this part of the QCL power stability measurement test apparatus. Both of these error sources could change with temperature, but we saw no evidence for reduced measurement variations after we stabilized the temperature of the $3 S^{3 i g m a}{ }^{\mathrm{TM}}$ by placing it in a Styrofoam cooler with a fan and a heat sink with temperature-stabilized water from the custom environmental chamber circulating through it on its way back to the circulator. Our procedure of measuring the power meter zero at the start of each measurement cycle and subtracting that reading from all the above threshold power readings minimizes the effects of zero drifts. Changes in gain of the electronics in the 3 Sigma ${ }^{\mathrm{TM}}$ or the power meter head would show up as a proportional change in the zero subtracted power readings. For example, a $1 \%$ increase in gain would increase a $6-\mathrm{mW}$ power reading by $0.06 \mathrm{~mW}$ while increasing a $29-\mathrm{mW}$ power reading by $0.29 \mathrm{~mW}$. We have seen no evidence of such proportional power reading variations. Figure 2.10 displays 10 days of data that show the most common behavior of variations, which include both variations that are independent of power level and variations that have nearly the same amplitude and time profile for all power levels.

\subsubsection{Output Resolution}

The digital output of the 3 Sigma ${ }^{\mathrm{TM}}$ readout on the 30 -mW full-scale setting, which was used for almost all of the power measurements in FY08, is $0.01 \mathrm{~mW}$. As discussed in the FY07 Calibrations report, this quantization limits the zero subtraction accuracy and contributes to the reading variations. This quantization contributes a root mean square (RMS) error of $2.9 \mu \mathrm{W}$ to each power reading and zero subtraction increases this RMS error by a factor of $\sqrt{2}$. The averaging of 10 readings per step that we do during data collection reduces this RMS error if the power and noise variations cause the quantized output to change among these readings. The amount of error reduction is at most a factor of 10, perhaps the square root of the number of independent measurements, or perhaps no reduction if there are no variations in the readings. The number of independent power readings given the 0.5 -second sampling interval and the 2-second response time of the power meter head is 2.5 . The value of $2.9 \mu \mathrm{W} \times \sqrt{2 / 2.5}$ or $2.6 \mu \mathrm{W}$ is close to the measurement noise we found in FY07. To reduce the error source, we tried using the analog output of the 3 Sigma ${ }^{\mathrm{TM}}$ and measuring it with an analog input on the NI-6221 data acquisition card. However, we found that the analog output is also quantized and is even a little more coarsely quantized than the digital output. It appears that the analog output is produced from a digital number by a digital-toanalog converter in the 3 Sigma ${ }^{\mathrm{TM}}$ readout. 


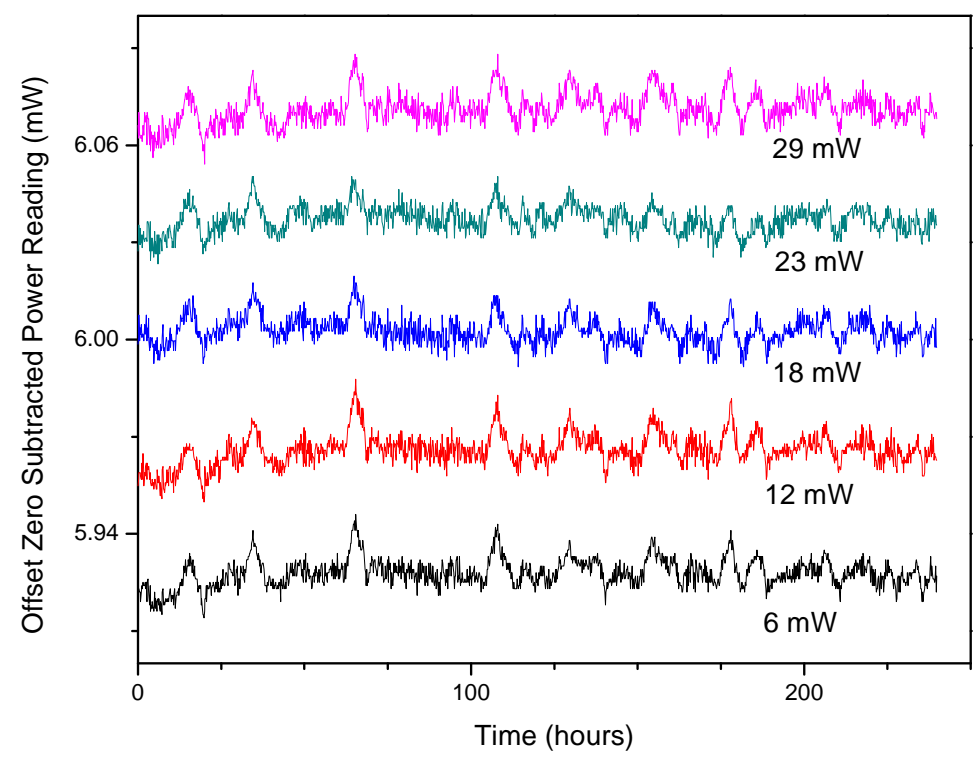

Figure 2.10. Variations in Power Readings over 10 Days at Argos QCL Output Powers of about 6, 12, 18, 23, and $29 \mathrm{~mW}$. There are smaller variations that are independent of power level, and there are common variations that have about the same amplitude and time profile for all power levels; but there is no evidence of variations that are proportional to the power levels. That is, there is no apparent variation in the 6-mW data that appears twice as large in the $12-\mathrm{mW}$ data, three times as large in the $18-\mathrm{mW}$ data, etc. up to five times as large in the $29-\mathrm{mW}$ data.

\subsubsection{Custom Readout Amplifier}

To reduce the quantization noise due to the digital output of the $3 \operatorname{Sigma}^{\mathrm{TM}}$, we built a custom lowdrift and low-noise amplifier that we connected to the signal output of the power meter head in parallel with the 3Sigma ${ }^{\mathrm{TM}}$ readout. The computer measured this amplifier's output with a 16-bit analog input on the NI-6221 data acquisition card that was usually used to measure the current monitor output from the QC5. This amplifier's voltage gain is 1000 and it was housed in the Styrofoam cooler housing the 3Sigma ${ }^{\mathrm{TM}}$ readout. Details of the amplifier are given in Appendix A.

Figure 2.11 compares the instrumental noise with the 3 Sigma $^{\mathrm{TM}}$ readout and with the custom readout amplifier for 10 days of data collection. The standard measurement cycle begins with two 15-secondlong steps with the current a little below threshold and finishes with a final 15-second step with the current off, which gives three zero readings. Differences between these zero readings provide a measure of the instrumental noise of the apparatus with a similar correction for the long-term zero drifts subtracted out as is used in the power stability data. Differences between the pair of initial zero readings, which are 15-seconds apart, give an estimate of the lower limit on the instrumental noise including drifts due to temperature changes while the differences between the final zero reading and the second zero reading are separated by 15 seconds more than that used for the highest power step. Figure 2.11 shows that there is little difference between these two differences in zero readings when using the 3 Sigma ${ }^{\mathrm{TM}}$ readout [traces a) and b)], and the standard deviations for these data are 2.4 and $2.5 \mu \mathrm{W}$. With the custom readout 
amplifier, where the relative quantization noise is ten times smaller, the standard deviations are lower, $0.49 \mu \mathrm{W}$ and $1.35 \mu \mathrm{W}$, and the initial zero differences [traces c) and d) in Figure 2.11] are much more stable. The initial zero-difference data corresponds to an $80-\mathrm{nV}$ standard deviation in the power meter head output. The conversion between amplifier output and power reading was determined by linear regression between the digital readings from the 3 Sigma $^{\mathrm{TM}}$ and the simultaneously measured amplifier voltages and was reproducible to about 10 parts-per-million over about 2 months.

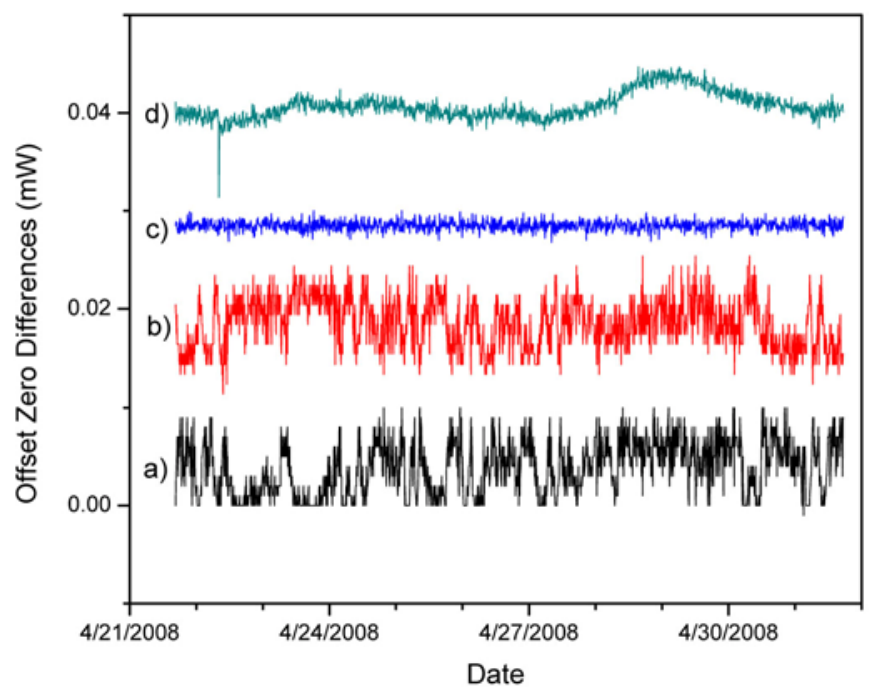

Figure 2.11. Zero Differences of 3 Sigma $^{\mathrm{TM}}$ Readout and Custom Readout Amplifier. These traces are from 10 days of data collection and are differences between "zero" readings either 15-seconds apart [a) and c)] or 105-seconds apart [b) and d)]. The lower two traces were measured with the 3 Sigma $^{\mathrm{TM}}$ readout and the upper two traces were measured using the custom readout amplifier and the NI-6221 analog input.

Figure 2.12 shows the zero-subtracted power readings at the $29-\mathrm{mW}$ step measured with both the 3 Sigma ${ }^{\mathrm{TM}}$ readout and the custom readout amplifier for the same data collection run as in Figure 2.11. The longer-term variations track each other well but there is less rapid variation with the custom amplifier which is reflected by the standard deviation of $3.5 \mu \mathrm{W}$ in comparison to the $4.4-\mu \mathrm{W}$ standard deviation for the 3 Sigma $^{\mathrm{TM}}$ data. This data supports the ideas that the 3 Sigma ${ }^{\mathrm{TM}}$ readout contributes significantly to the short-term variations in the power readings and that the longer-term variations seen in Figure 2.12 are due to changes in the output of the power meter head and not the readout.

\subsubsection{Measurement System Noise}

The standard measurement cycle consists of eight 15-second-long steps with the first two having the current through the QCL held at levels slightly below threshold, five with the current above threshold, and a final step with the current at zero. Power readings for the second step are used for the power meter zero readings for each cycle. By looking at the zero-subtracted power readings for the last step, we can estimate the variability that is due to the measurement system without the variations due to changes in power from the QCL. Figure 2.13 shows the standard deviations for these data as a function of measurement duration. 


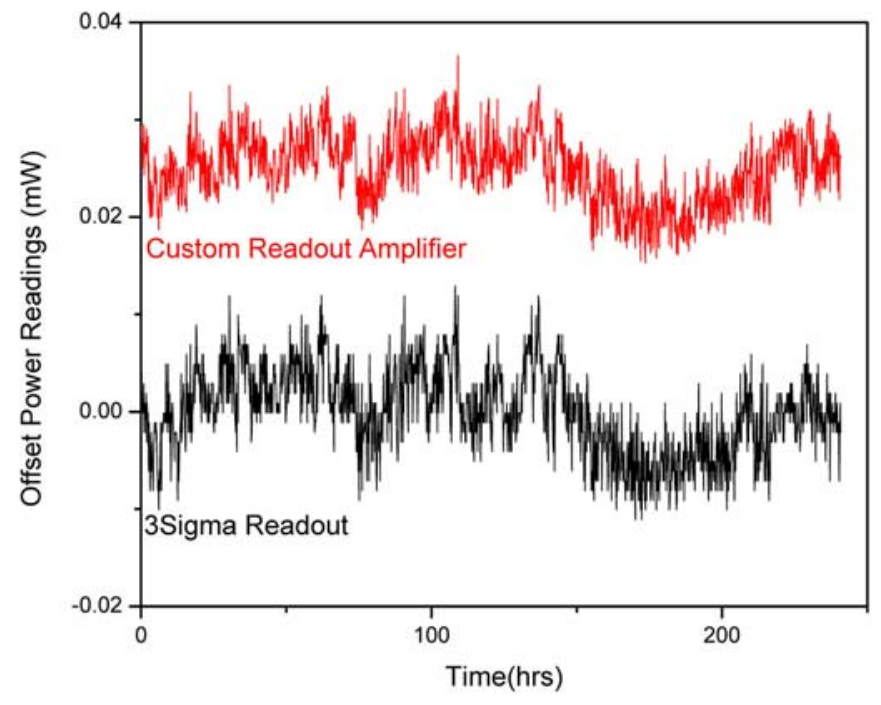

Figure 2.12. Correspondence of Power Readings with 3 Sigma ${ }^{\mathrm{TM}}$ Readout and Custom Readout Amplifier for 29-mW Power Level

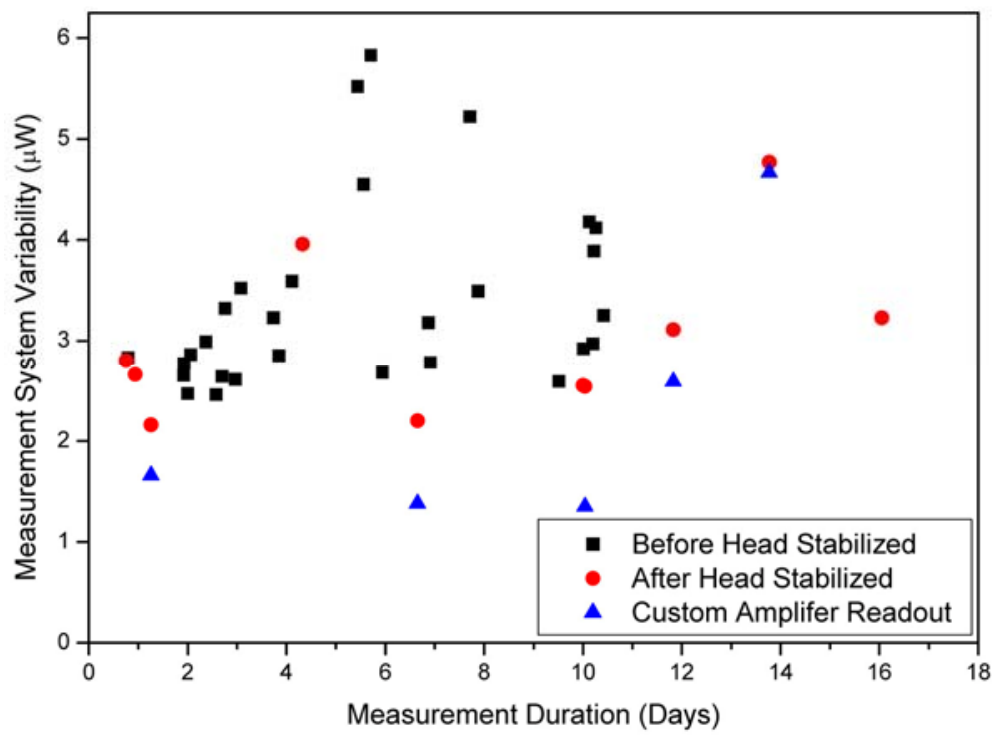

Figure 2.13. Variability of Measurement System Readings as a Function of Measurement Duration. After the power meter head temperature was stabilized, the maximum system variability was reduced making it more likely that this variability would be $3 \mu \mathrm{W}$ or less; that is, $0.01 \%$ of full scale. The custom amplifier-based readout can significantly reduce measurement variability compared to the $3 \mathrm{Sigma}^{\mathrm{TM}}$ readout unless the power meter head zero is changing on a time scale of a couple of minutes. 



\subsection{Device Performance}

\subsection{Argos QCL}

In FY08, a QCL manufactured by Argos, a spin-out company from Agilent, was a major focus of measurements. This QCL had a buried heterostructure design, which provided efficient heat removal and contributed to its relatively low room temperature threshold current for cw operation. This Fabry-Perot QCL was mounted in an "epi-up" configuration on a modification of the commercial C-mount for diode lasers and had a high reflectance coating on the back facet. Figure 2.4 shows an optical power versus current plot at $20^{\circ} \mathrm{C}$ for this laser that has a threshold current about three times smaller than the QCLs we have from Maxion that operate $\mathrm{cw}$ at room temperature. This QCL has a maximum of about $100 \mathrm{~mW}$ in $\mathrm{cw}$ operation at room temperature and lases around 8.7 microns.

\subsubsection{Uncollimated Argos QCL Stability}

We started power stability measurements on the Argos QCL at the end of FY07 and found power variations in cw operation that change with bringing the QCL above threshold. These approximately $0.5 \%$ variations for all power levels were eliminated upon switching to using 50\% duty cycle, $100 \%$ amplitude modulation at $40 \mathrm{kHz}$ for the current as discussed in the FY07 Calibrations report (Myers et al. 2007). Figure 3.1 shows the zero-subtracted power readings at all five levels for the first month that the modulated current waveform was used to power this QCL. The five traces show parallel changes of close to the same magnitude, which could reflect changes in laser threshold or changes in an electronic offset in the control system or current controller. The large change at d) in Figure 3.1 corresponds to a power decrease of $0.14 \mathrm{~mW}$ or just over $2 \%$ of the $6-\mathrm{mW}$ power level. This corresponds to an offset in the currents of $0.34 \mathrm{~mA}$ or an offset in the voltage controlling the QC5 current supply of $3.4 \mathrm{mV}$. The largest source of variation is the experimenter trying to improve the stability.

Figure 15 shows the data collected through the end of 2007 following the nearly 4-week hiatus to implement the software changes needed to simultaneously measure the QCL compliance voltage and power when using the modulated current. During this hiatus, the power levels from the Argos QCL changed only $-0.023 \pm 0.035 \mathrm{~mW}( \pm 2 \times$ Std. Dev.), which may reflect changes in average laboratory temperature or other changes in the test apparatus. As in Figure 3.1, the major source of variation in the power readings is the experimenter changing the apparatus in attempts to improve the stability. The increase at all power levels between a) and b) in Figure 15 was traced to accidentally touching the coarse current control potentiometer on the QC5 while striving to improve power stability. Between c) and d) in Figure 15 can be seen a rare instance of a change of the power meter calibration when the 3 Sigma $^{\mathrm{TM}}$ overheated as a result of inadequate air flow when trying to stabilize its temperature. Starting at e) in Figure 15, the 3 Sigma $^{\mathrm{TM}}$ was temperature stabilized, although there was no reduction in the variations in zero readings and zero-subtracted power readings due to laboratory temperature changes. There are shifts in the power levels of $0.065,0.065,0.068$, 0.026 , and $0.037 \mathrm{~mW}$ for the $6-\mathrm{mW}$ to $29-\mathrm{mW}$ power levels between the initial and final data in Figure 15 . This power shift might in part reflect the effect of overheating the 3 Sigma $^{\mathrm{TM}}$ and in part reflect changes in the QC5 temperature due to re-positioning components and adding the water-cooled heat exchanger for the 3 Sigma ${ }^{\mathrm{TM}}$ into the cooling loop. As of the end of the first quarter of FY08, the only way identified to improve the stability of the power readings is for the experimenter to not try to improve anything! 


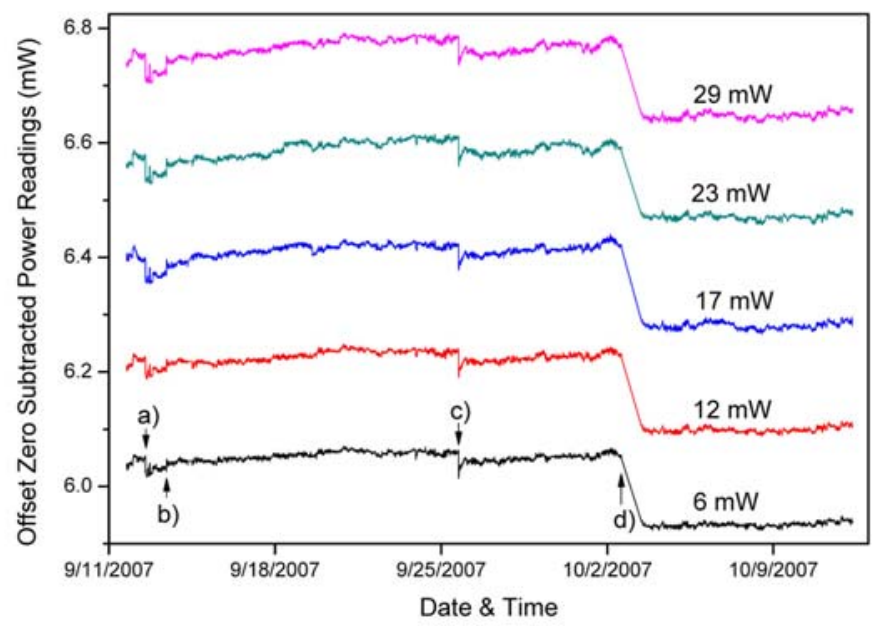

Figure 3.1. First Month of Data from the Argos QCL Using the Modulated Excitation. The abrupt changes marked with letters correspond to the experimenter changing something in the testing apparatus. a) Start experimenting with current monitor to fix 2-MHz oscillation. b) Installed filter on current monitor that fixed oscillation. c) Wired Agilent DMM in series to measure current and then removed Agilent from circuit and re-started data collection. d) Replaced interface box between NI-6221 and instruments and then changed from singleended to differential input from the current monitor filter output. Opened custom environmental chamber to install cable for 4-wire measurement of QCL compliance voltage that was also connected in a differential input to the NI-6621. Finally, changed the analog output from the NI-6221 from single ended to differential.

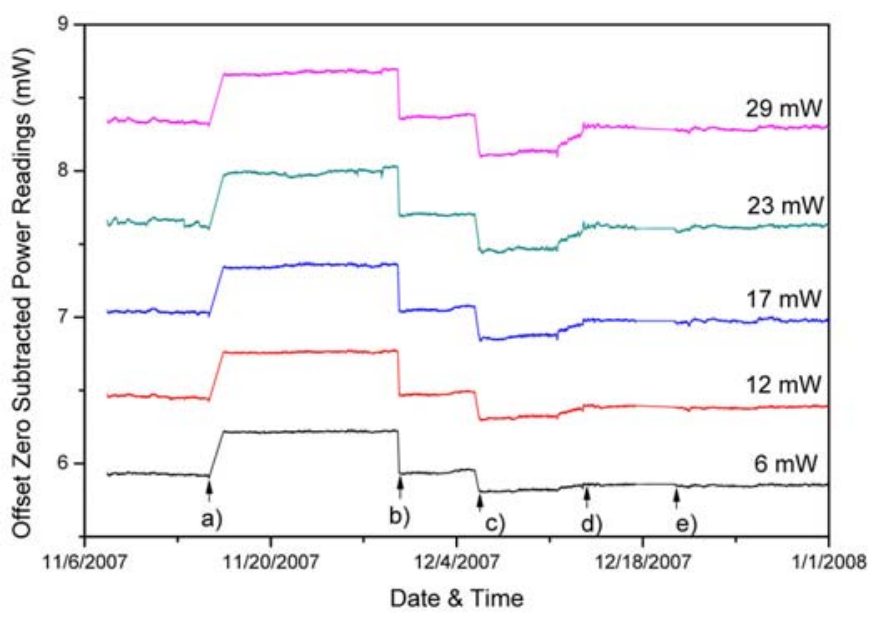

Figure 3.2. Argos Power Readings from the Initial Ability to Measure QCL Compliance Voltage While Using the Modulated Current Waveform Until the End of 2007. The shift in the power readings from the end of the data in Figure 3.1 and the beginning of the data in this figure is $-0.023 \pm 0.035 \mathrm{~mW}$ for the five levels. a) Accidentally turned coarse current control potentiometer very slightly while untangling cables and re-positioning QC5 in rack. b) Discovered coarse current adjust was rotated and fixed by removing the potentiometers from the circuit. Moved 3Sigma ${ }^{\mathrm{TM}}$ power meter readout to try to stabilize its temperature. c) 3 Sigma ${ }^{\mathrm{TM}}$ overheated due to insufficient air flow. d) 3 Sigma $^{\mathrm{TM}}$ finished cooling off. e) $3 S_{i g m a}{ }^{\mathrm{TM}}$ in separate Styrofoam cooler with fan and water cooled heat exchanger. 
Figure 3.3 shows the Argos power stability data for the second quarter of FY08. The 2 months from 12/27/2007 until 2/26/2008, labeled d) in Figure 3.3, is the longest stretch of data for the Argos QCL where the test apparatus was not significantly changed. There was a 2-day period between b) and c) in Figure 3.3 when the temperature of the cooling water was changed from $22.0^{\circ} \mathrm{C}$ to $23.0^{\circ} \mathrm{C}$ and then back to investigate the cause of the power meter zero fluctuations due to laboratory temperature changes. However, the corresponding changes in power are small and will not substantially increase the standard deviations of these data. Table 3.1 summarizes the results of this stability data with twice the relative standard deviations for these 2 months ranging from $0.22 \%$ at the lowest power level to $0.11 \%$ at the highest power level. Linear least-squares fits of these data gave slopes that are all positive although small. These slopes are given in Table 3.1 as both power change per day of measurement and as power change per hour the QCL was on; that is, current was flowing. The standard deviations from the linear fits for these data are slightly lower than the standard deviations given in Table 3.1, but decreases range from $13 \%$ at the lowest power level to $26 \%$ at the highest level.

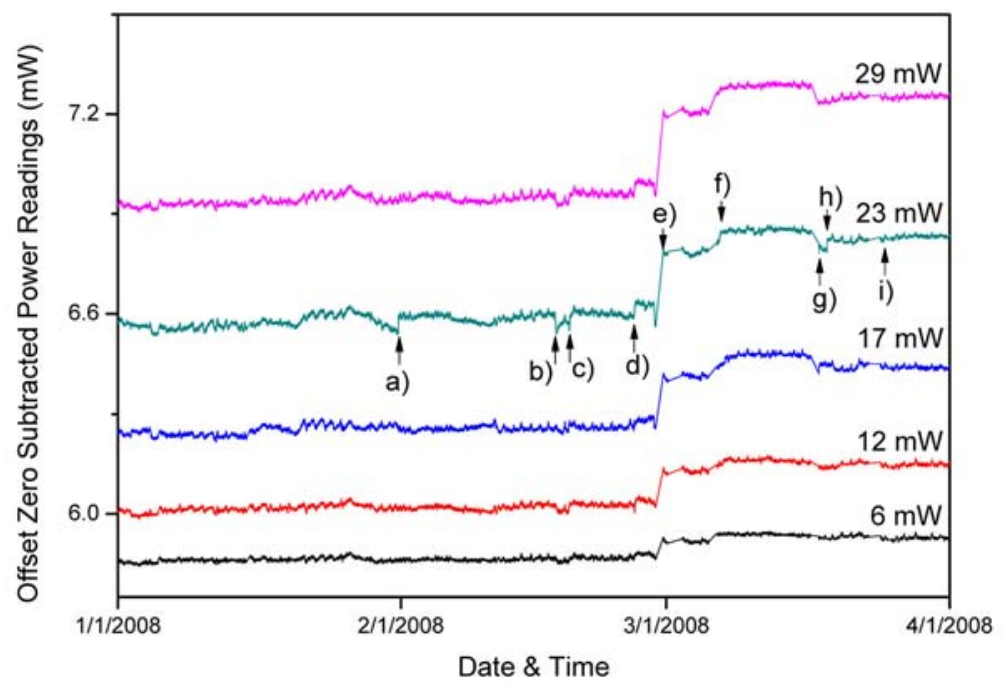

Figure 3.3. Argos Power Readings for the Second Quarter of FY08. This data includes the longest period free of substantial interference by the experimenter and the period when the variations in the power meter zero readings changing with laboratory temperature changes were traced to changes in power meter head temperature. a) A rare example of a rapid shift in power that is only seen for one power level and has no obvious correlation with any change caused by the experimenter. b) and c) Beginning and end of interval when the circulating water temperature was changed from $22.0^{\circ} \mathrm{C}$ to $23.0^{\circ} \mathrm{C}$ and then back to $22.0^{\circ} \mathrm{C}$ to diagnose mechanism of power meter zero variations. d) Installed external platinum resistance thermometer (PRT) in water return line from back plate on QCL mount and started using it for the temperature control of the circulating water. e) Installed the first version of the cooling loop onto the power meter head. f) Moved external PRT from return line to input line going into cooling loop on power meter head. g) Installed improved cooling loop onto the power meter head and wrapped head and cooling loop in aluminum foil. h) Another case of a rapid shift in power for the $23 \mathrm{~mW}$ that is not reflected in any of the other power levels and not correlated with any known change by the experimenter. i) Re-started data collection after computer hung due to problem reading from ILX temperature controller and after cycling the power on the ILX and re-booting the computer. 
Table 3.1. Power Stability of the Two Months from 12/27/2007 Until 2/26/2008 of the Uncollimated Argos QCL after Stabilizing the Temperature of the 3 Sigma $^{\mathrm{TM}}$ Readout but before Stabilizing the Temperatures of the QC5 Current Controller and Improving the Temperature Stabilization of the Power Meter Head

\begin{tabular}{lccccc}
\hline & $\mathbf{6 ~ m W}$ & $\mathbf{1 2} \mathbf{~ m W}$ & $\mathbf{1 7} \mathbf{~ m W}$ & $\mathbf{2 3} \mathbf{~ m W}$ & $\mathbf{2 9} \mathbf{~ m W}$ \\
Level & Level & Level & Level \\
\hline Average Power $(\mathrm{mW})$ & 5.862 & 11.684 & 17.349 & 22.929 & 28.532 \\
Std. Dev. $(\mu \mathrm{WW})$ & 6.4 & 10.0 & 12.3 & 17.5 & 15.2 \\
$2 \times$ Rel. Std. Dev. & $0.22 \%$ & $0.17 \%$ & $0.14 \%$ & $0.15 \%$ & $0.11 \%$ \\
Slope $(\mu \mathrm{W} /$ day) & 0.18 & 0.32 & 0.36 & 0.58 & 0.58 \\
Slope $(\mathrm{nW} / \mathrm{hr}$ on) & 42 & 77 & 85 & 140 & 140 \\
\hline
\end{tabular}

The power drifts could be due to changes in the QCL, changes in the QCL temperature, changes in current through the QCL, or changes in the power meter. We have simultaneous data from the current monitor of the QC5 and the QCL compliance voltage for the period 1/2/2008 until 2/4/2008. Before and after these dates, the current monitor was disconnected in attempts to find the sources of variation, which did not appear to affect the power stability, but did change the QCL voltage readings. This interaction between these two sets of readings may be due to cross-talk between different analog input channels of the NI-6221. With only $12 \mu$ s between a current reading and the subsequent QCL voltage readings, the ADC input may retain some memory of the previous value as the multiplexer in the NI-6221 switches between channels. Linear regression on the magnitudes of the current monitor, QCL voltage, and power data in this window gives small positive slopes. Using the $0.41-\mathrm{mW} / \mathrm{mA}$ slope from Figure 2.4 and the 5.3-ohm differential resistance of the Argos QCL in the range of currents used, these slopes from the regression on the current monitor and QCL voltage data can be converted to predicted power drift rates if changes in current are responsible for the drift in power. Figure 3.4 shows the measured drift in the power readings as well as that expected based on the current monitor and QCL voltage readings assuming a change in current is responsible for the drifts. The current monitor and QCL voltage readings indicate drift rates that are independent of the power level and hence the current level. However, the drift rate of the current monitor readings is almost ten times larger than would be expected from the QCL voltage readings. In contrast, the power reading drift rate increases almost linearly with average power level and is bracketed by the predictions from the QCL voltage and the current monitor readings. It appears that the current monitor readings are drifting at a much higher rate than the actual change in the current through the QCL. In addition, the increase in power readings does not appear to be related to a change in current through the QCL. From Table 2.1 we see that the change in QCL power with heat-sink temperature changes from $-0.78 \mathrm{~mW} /{ }^{\circ} \mathrm{C}$ at about $6 \mathrm{~mW}$ to $-0.98 \mathrm{~mW} /{ }^{\circ} \mathrm{C}$ at about $29 \mathrm{~mW}$. This $25 \%$ increase between the $6 \mathrm{~mW}$ and $29 \mathrm{~mW}$ is not consistent with the drift rates observed in the power readings. Thus, the drift in power readings is not consistent with a small temperature drift in the QCL. Changes in the power meter offsets are subtracted out each cycle to about $\pm 0.005 \mathrm{~mW}$ as shown in traces a) and b) of Figure 2.11. So changes in the power meter should extrapolate to zero drift rate at zero average power. The measured power reading drift rates shown in Figure 3.4 might be compatible with this behavior. The drift rates in Table 2.1, which are for data covering twice the time span as those in Figure 3.4, are 50-70\% of those in Figure 3.4 and are better fit by a line through the origin. A constant drift rate of the threshold of the QCL would give a power drift rate independent of average power while a constant drift rate in the slope efficiency of the QCL would give a power drift rate linear in the average power. Thus, changes in 
the power meter or changes in the QCL itself are consistent with the data in Figure 3.4, but changes in current or the QCL temperature are not.

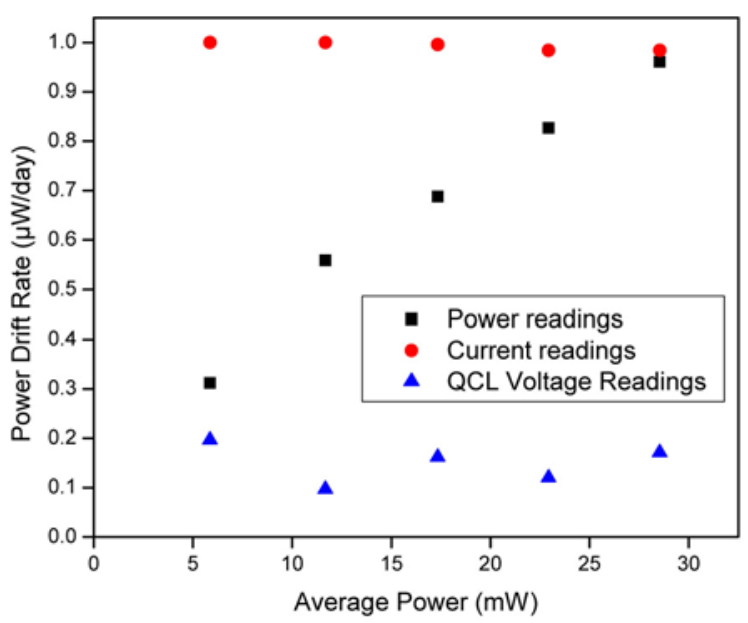

Figure 3.4. Drift Rates in Power Readings from January 2, 2008, to February 4, 2008, and Predicted Power Reading Drift Rates for the Argos QCL Based on Measured Current Monitor and QCL Voltage Drift Rates

Between points d) and g) in Figure 3.3, we made several changes to improve the temperature stability of the power meter head, which caused shifts in power readings that are approximately proportional to the average power. This is at least qualitatively consistent with the model that the improved thermal contact with the power meter head results in an approximately $1 \%$ increase in the temperature difference across the thermopile for a given incident power.

Figure 3.5 shows the power reading data from the 3 Sigma $^{\mathrm{TM}}$ after the changes to improve the power head temperature stability were finished until the end of data collection from the uncollimated Argos QCL. The 10 days between a) and b) seemed to indicate improved stability resulted from the improvements in the power meter head temperature stability. Table 3.2 summarizes these results, with the largest improvement being that the standard deviations are essentially independent of the power level rather than increasing almost three times as shown in Table 3.1 for the two highest power levels. Another notable change is that the power readings are almost $1 \%$ higher at all levels, which may reflect faster heat removal from the reference side of the thermopile.

The period between c) and e) in Figure 3.5 is when we experimented with using a custom amplifier in parallel with the 3 Sigma $^{\mathrm{TM}}$ to reduce the effects of the $\pm 0.005-\mathrm{mW}$ quantization error of the 3 Sigma ${ }^{\mathrm{TM}}$, $\mathrm{s}$ output. Unfortunately, impatience led us to start thermally stabilizing the QC5 current controller after about a week, which makes it unclear if the slow decline in power readings is due to the custom readout amplifier in some way or to having moved the QC5 into the Styrofoam cooler with the 3Sigma ${ }^{\mathrm{TM}}$. The failure of the downward slope to continue after the custom amplifier was removed at e) suggests that the amplifier was involved. However, it is puzzling that the power readings did not return to the levels at d) and in fact dropped further, with the magnitude of the power drops increasing with the average power level. 


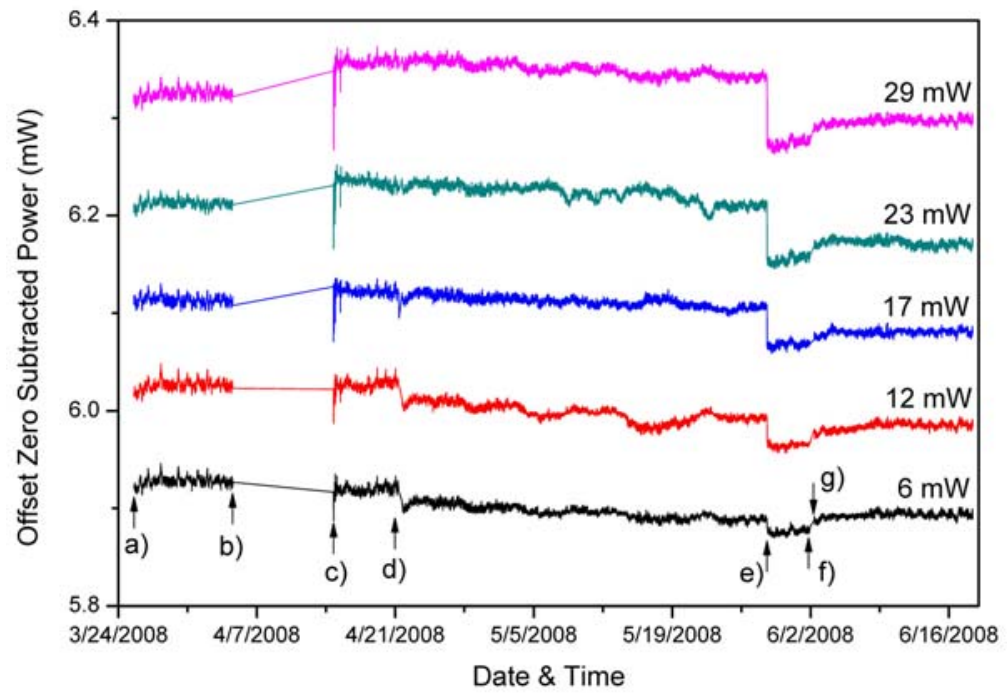

Figure 3.5. Power Readings Using the 3 Sigma $^{\mathrm{TM}}$ Readout for the Uncollimated Argos QCL After the Improved Stabilization of Power Meter Head Temperature. a)-b) Head temperature stabilized and custom readout amplifier not yet installed. c) Installed debugged custom readout amplifier, which required disconnecting the current monitor from the NI-6221. On starting data collecction, the power meter zero was very unstable and remnants of this instability can be seen in the zero-subtracted power readings. d) Moved QC5 current controller into Styrofoam cooler with 3 Sigma ${ }^{\mathrm{TM}}$ readout and custom readout amplifier.

e) Removed custom readout amplifier and re-connected the current monitor to the NI-6221. f) Computer hung due to error from ILX temperature controller. g) Re-started data collection after cycling power on ILX temperature controller and re-booting computer.

Table 3.2. Power Stability for the 10 Days Between a) and b) in Figure 3.5 After Improving the Power Meter Head Temperature Stability

\begin{tabular}{lccccc}
\hline & $\begin{array}{c}\mathbf{6} \mathbf{~ m W} \\
\text { Level }\end{array}$ & $\begin{array}{c}\mathbf{1 2} \mathbf{~ m W} \\
\text { Level }\end{array}$ & $\begin{array}{c}\mathbf{1 7} \mathbf{~ m W} \\
\text { Level }\end{array}$ & $\begin{array}{c}\mathbf{2 3} \mathbf{~ m W} \\
\text { Level }\end{array}$ & $\begin{array}{c}\mathbf{2 9} \mathbf{~ m W} \\
\text { Level }\end{array}$ \\
\hline Average Power $(\mathrm{mW})$ & 5.928 & 11.815 & 17.535 & 23.181 & 28.843 \\
Std. Dev. $(\mu \mathrm{W})$ & 4.4 & 4.9 & 4.2 & 4.1 & 4.9 \\
$2 \times$ Rel. Std. Dev. & $0.15 \%$ & $0.083 \%$ & $0.048 \%$ & $0.035 \%$ & $0.034 \%$ \\
\hline
\end{tabular}

Some insights into these puzzles can be obtained from the QCL compliance voltages shown in Figure 3.6, which were recorded simultaneously with the power readings in Figure 3.5. While there are offsets between b) and c) in both Figure 3.5 and Figure 3.6, the directions of the offsets as well as the relative magnitudes are different between the compliance voltages and the power readings. Thus, these offsets are not due solely to changes in the current through the QCL. However, a simple model can explain these data. If we assume that the change in the measured QCL compliance voltage is due to an offset that is independent of the power level, $V_{\text {offset }}$, and a change in current through the QCL that may depend on the power level, $\Delta I(P)$, then the change in compliance voltage, $\Delta V(P)$ would be

$$
\Delta V(P)=V_{\text {offset }}+5.29 \Omega \cdot \Delta I(P)
$$




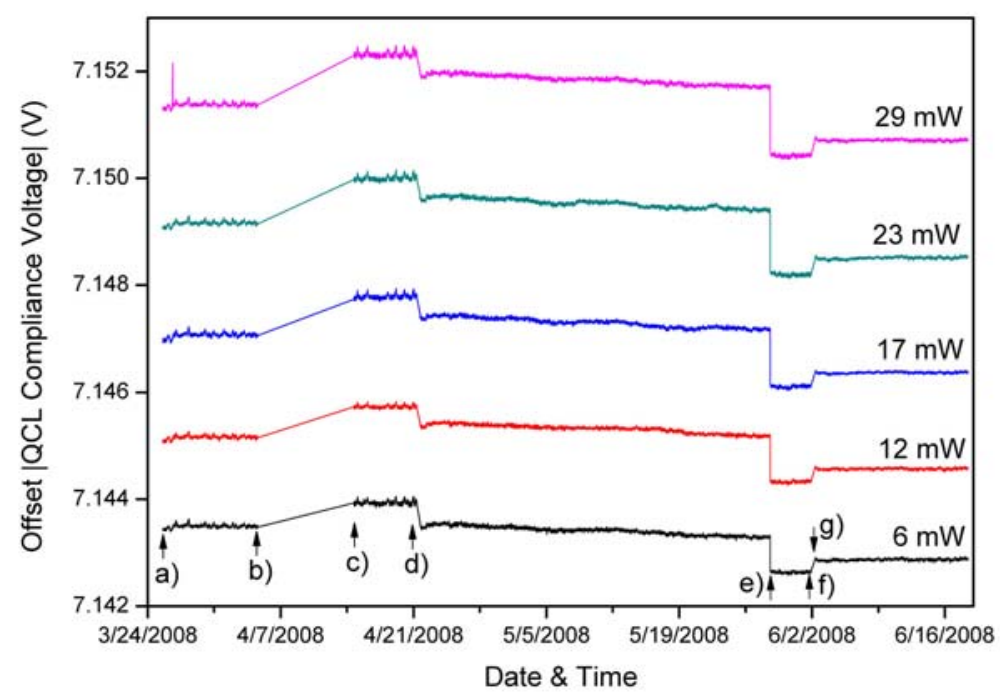

Figure 3.6. Magnitudes of the QCL Compliance Voltages for Each Power Level Recorded Simultaneously with the Power Readings in Figure 3.5. The points a)-g) have the same meanings as in Figure 3.5. The spike on the 29-mW level trace on 3/24/2008 resulted from plugging something into the DC power supply that powers the QC5 current controller.

where the $5.29 \Omega$ measured differential resistance of the Argos QCL has been used. Then using the $0.41-\mathrm{mW} / \mathrm{mA}$ slope from Figure 2.4, the change in power, $\Delta P(P)$ for each power level would be

$$
\Delta P(P)=0.41(m W / m A) \cdot \Delta I(P)=\left(\frac{0.41(m W / m A)}{5.29 \Omega}\right) \cdot\left(\Delta V(P)-V_{\text {offset }}\right) .
$$

The single adjustable parameter in this model is $V_{\text {offset }}$. Figure 3.7 a shows the good agreement between this model and the measured power offsets using $-0.58 \mathrm{mV}$ for $V_{\text {offset }}$ and the differences between the 24-hour averages of the power and compliance voltages before point b) and after point c) in Figure 3.5 and Figure 3.6. This value for $V_{\text {offset }}$ is approximately twice the least significant bit of the analog input on the $\pm 10 \mathrm{~V}$ range of the NI-6221 used for this measurement. The small residual differences are probably due to the higher noise at point c) because of power meter zero instability. The physical cause of $V_{\text {offset }}$ could be grounding issues, contact potential changes, and/or cross talk in the NI-6221 input that arise from disconnecting the current monitor and connecting the custom amplifier to the NI-6221 and connecting the custom amplifier to the power meter head. We do not have an explanation for the cause of the +29 to $-67 \mu$ A changes in the QCL current that are implied by this model. However, it does not seem likely that changes in the QCL need be invoked to explain this data. 

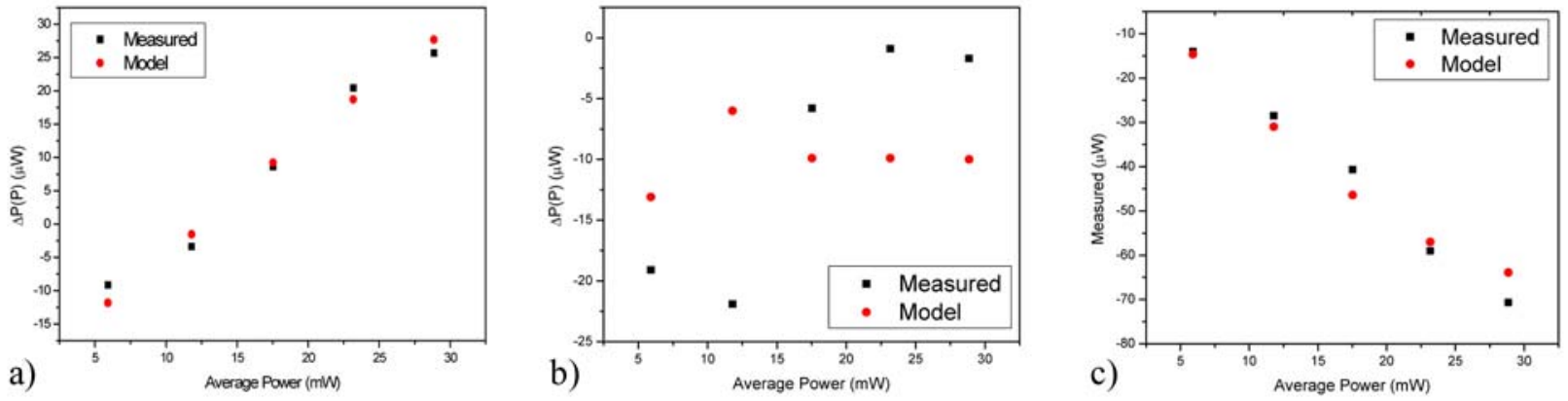

Figure 3.7. Model Matching Measured Changes in Powers as a Function of Average Power, $\Delta \mathrm{P}(\mathrm{P})$ to a Small Voltage Offset and the Measured Compliance Voltage Differences. a) For the changes between points b) and c) in Figure 3.5. The largest difference between the model and data is $3 \mu \mathrm{W}$. b) For the changes at point d) in Figure 3.5. c) For the changes at point e) in Figure 3.5. The largest difference between the model and the data is $7 \mu \mathrm{W}$.

Point d) in Figures 3.5 and 3.6 corresponds to moving the QC5 current controller into the Styrofoam cooler with the 3 Sigma ${ }^{\mathrm{TM}}$ readout and certainly resulted in a change in the temperature of the QC5. Disconnecting and re-connecting cables as part of this move could possibly have changed contact potentials and so changed the offset for the compliance voltage measurements. Figure 3.7b shows that the same model, with an optimized value of $V_{\text {offset }}$ of $0.28 \mathrm{mV}$, does not fit as well as for the changes between b) and c); with a maximum deviation of the model from the data of $16 \mu \mathrm{W}$. The variation in the power changes for this modification to the setup appear to indicate some additional process needs to be included in our simple model, but no process suggests itself. Figure 3.7c shows the changes at point e) in Figures 3.5 and 3.6 are also well described by this model with $V_{\text {offset }}$ of $+0.46 \mathrm{mV}$. For the changes between f) and g) in Figures 3.5 and 3.6 where the computer was rebooted and the power to the ILX temperature controller was cycled, this model fits well with a maximum deviation of $4 \mu \mathrm{W}$ and the offset voltage required is only $7 \mu \mathrm{V}$. This low value of $V_{\text {offset }}$ is to be expected given the very minor change involved in cycling the power on the ILX temperature controller and rebooting the computer.

The final common feature of Figures 3.5 and 3.6 is the negative slope between d) and e). If the changes in the compliance voltages are solely due to changes in current through the QCL, a plot of the power readings versus the magnitude of the compliances voltages for each average power level should have a slope of $1000 \times 0.415 \mathrm{~mW} / \mathrm{mA} / 5.29$ ohms or $78.5-\mathrm{mW} /$ volt. Table 3.3 shows the results of such an analysis using both the power readings from the 3 Sigma $^{\mathrm{TM}}$ readout and the custom amplifier. Except for the middle power level of $17.5 \mathrm{~mW}$, all the slopes are in good agreement with the predicted value, and the standard deviations from the fits are similar to the standard deviations we find for other power stability data from this QCL. It is interesting to note that the best agreement with the predicted slope and the smallest deviations from the fit are for power readings using the custom amplifier and with the shortest time between zero readings and power readings. It is puzzling that the data for the middle power level give slopes that are about $40 \%$ lower than predicted while for the other data the slopes match the predicted to within about $15 \%$. 
Table 3.3. Slopes of Plots of Power Readings Versus QCL Compliance Voltage for the Data Between d) and e) in Figures 3.5 and 3.6. Both the power readings using the $3 S_{i g m a}{ }^{\mathrm{TM}}$ power readout and the custom amplifier readout were analyzed.

\begin{tabular}{lccccc}
\hline Average Power $(\mathrm{mW})$ & 5.896 & 11.787 & 17.534 & 23.191 & 28.867 \\
Slope w/ 3Sigma ${ }^{\mathrm{TM}}$ Data $(\mathrm{mW} / \mathrm{V})$ & 74.7 & 75.2 & 45.6 & 70.8 & 69.7 \\
Std. Dev. from Fit $(\mu \mathrm{W})$ & 2.9 & 6.5 & 3.5 & 6.3 & 3.4 \\
Slope w/ Custom Amplifier Data $(\mathrm{mW} / \mathrm{V})$ & 78.4 & 91.5 & 48.3 & 73.8 & 77.3 \\
Std. Dev. from Fit $(\mu \mathrm{W})$ & 2.0 & 6.5 & 3.2 & 6.5 & 3.0 \\
\hline
\end{tabular}

In conclusion, most of the variations seen in Figures 3.5 and 3.6 can be explained as sub-mV changes in an offset to the QCL compliance voltage measurements and changes in current through the QCL that are reflected as changes in the QCL compliance voltages. It is not clear what causes all of these current changes although the ones that are discrete and happen when changing the measurement setup are likely because of a changed offset to the control voltage applied to the QC5. It is not clear what process could cause a decrease in the current through the QCL to produce the sloped regions in Figures 3.5 and 3.6 that would continue for about a month! A month seems too long for it to be due to a temperature change, and it is unclear why much of the decrease remained after the custom amplifier was removed.

The data after g) in Figure 3.5 when both the power meter head and the QC5 current controller were temperature stabilized and the custom amplifier was removed showed the best stability we have measured over more than a couple of days. Table 3.4 summarizes these results. The standard deviations of these data are very close to the system measurement noise of $2.7 \mu \mathrm{W}$ for the differences between the initial subthreshold steps and $3.2 \mu \mathrm{W}$ for the zero-subtracted last step data. It is interesting to note that the standard deviations of these data do not increase with average power and hence delay between the power reading and the zero measurement in contrast to Table 3.1 where the standard deviations increased from $6.4 \mu \mathrm{W}$ to $15 \mu \mathrm{W}$. The magnitudes of the slopes in Table 3.4 are similar to those in Table 3.1, but one slope is negative in the June 2008 data.

Table 3.4. Power Stability of the 16 Days from 6/2/2008 Until 6/18/2008 of the Uncollimated Argos QCL After Stabilizing the Temperature of the 3 Sigma $^{\mathrm{TM}}$ Readout, Stabilizing the Temperatures of the QC5 Current Controller, and Improving the Temperature Stabilization of the Power Meter Head

\begin{tabular}{lccccc}
\hline & $\mathbf{6 ~} \mathbf{~ m W}$ & $\mathbf{1 2} \mathbf{~ m W}$ & $\mathbf{1 7} \mathbf{~ m W}$ & $\mathbf{2 3 ~} \mathbf{~ m W}$ & $\mathbf{2 9} \mathbf{~ m W}$ \\
Level & Level & Level & Level & Level \\
\hline Average Power $(\mathrm{mW})$ & 5.893 & 11.773 & 17.503 & 23.140 & 28.815 \\
Std. Dev. $(\mu \mathrm{W})$ & 2.9 & 3.8 & 2.8 & 3.6 & 3.4 \\
$2 \times$ Rel. Std. Dev. & $0.099 \%$ & $0.065 \%$ & $0.032 \%$ & $0.031 \%$ & $0.023 \%$ \\
Slope $(\mu \mathrm{W} /$ day) & 0.23 & 0.46 & 0.15 & -0.30 & 0.29 \\
Slope $(\mathrm{nW} / \mathrm{hr}$ on) & 55 & 109 & 35 & -71 & 68 \\
\hline
\end{tabular}




\subsubsection{Collimated Argos QCL}

With the excellent stability of the uncollimated Argos QCL established, we investigated how the stability would be affected by collimating the light from this QCL. This QCL was mounted on the optics sub-platform developed for the high heat load package in another project at PNNL (Anheier et al. 2008). The collimating lens is an aspheric design with a diameter of $5 \mathrm{~mm}$ and a numerical aperture (NA) of 0.85. We assembled a second "custom environmental chamber" based on what we learned from the first setup and re-using the computer and some instruments from the cryogenic QCL test setup. There are four main differences between the new setup and the one used for the work on the uncollimated Argos QCL:

1. The current controller is a model QC6N, which has a maximum output of 2 amperes rather than 1 ampere for the QC5, and the transfer function of the external input is $200 \mathrm{~mA} /$ volt rather than $100 \mathrm{~mA} /$ volt.

2. The power meter head was about 3 inches from the QCL mount, which resulted in the field of view of the power meter head not being dominated by the gold-coated cold plate of the QCL mount, but included much of the new laser mount and some of the walls of the plastic tub that was the inner chamber.

3. The mass of material that was temperature controlled along with the QCL was substantially increased from just the C-mount to the C-mount and the optics sub-platform and an aluminum plate. This larger thermal mass slowed down the response time of the temperature stabilization feedback loop.

4. The power meter head, newly returned from servicing and re-calibration, has a 10 -mm-diameter sensitive area rather than the 19-mm diameter in the other setup.

During the initial testing, we wired the Agilent digital multimeter (DMM) in series between the QC6N and the Argos QCL. For four measurement cycles, separated by 10 minutes, 3.83 hours, and 14.17 hours, we recorded current readings from the Agilent $61 / 2$-digit DMM. The standard deviations of these readings were approximately $6 \mu \mathrm{A}$ at all eight steps. This Agilent data was used to calibrate the current monitor output of the QC6N, which gave a slope of $1.0073 \pm 0.0002$ and an intercept of $4.74 \pm$ $0.03 \mathrm{~mA}$.

Measuring the optical power of the collimated Argos QCL versus twice the current monitor readings (approximately the peak current values) gave a slope of $0.544 \pm 0.005 \mathrm{~mW} / \mathrm{mA}$. This slope is $30 \%$ larger than we measured with this QCL before collimation. Correcting for the slightly different calibrations of the current monitor outputs of the QC5 and QC6N current controllers makes about a 1\% difference in the slopes. This increased slope probably reflects more of the laser emission being collected by the 0.85-NA lens than by the 19-mm-diameter sensitive area of the power meter head. Including corrections for the offsets in the current monitor readings for both controllers, the threshold for the Argos QCL before collimation is $267.2 \mathrm{~mA}$ while for the collimated Argos QCL, the corrected threshold is $281.8 \mathrm{~mA}$. The collimated Argos has a threshold about $15 \mathrm{~mA}$ higher than before collimation. This could reflect the difference between the true temperatures of the Argos QCL in the two setups due to errors in the thermistor calibrations and the differences in heat sinking of the QCL for the two mountings. However, it is also possible the feedback from the lens is also partially responsible for the increase in both threshold current and L-I slope. 


\subsubsection{Performance of Second Test Setup and Collimated Argos QCL}

For the 14.6 days of data on the collimated Argos QCL, the system measurement is $1.4 \mu \mathrm{W}$ using the difference between the two initial sub-threshold steps and $2.7 \mu \mathrm{W}$ using the zero-subtracted last readings. This is comparable or a little better than the original setup. Table 3.5 summarizes these stability measurements, and at the highest power level the variations are 2.4 times larger than in Table 3.4. For the two highest power levels, the average drifts of the power readings are $7.9 \mathrm{nW} /$ cycle and $8.7 \mathrm{nW} /$ cycle, while the compliance voltage drifts are $83 \mathrm{nV} /$ cycle and $87 \mathrm{nV} /$ cycle, which give ratios of $94 \mathrm{~mW} / \mathrm{V}$ and $100 \mathrm{~mW} / \mathrm{V}$. If current changes are responsible for these drifts, then these ratios should be $0.544 \mathrm{~mW} / \mathrm{mA}$ $\times 1000 / 5.29 \mathrm{ohms}$, which is $103 \mathrm{~mW} / \mathrm{V}$. Similarly, the drifts for the current monitor readings for these two levels are both $19 \mathrm{nA} /$ cycle, and the ratios to the power reading drifts gives $0.42 \mathrm{~mW} / \mathrm{mA}$ and $0.46 \mathrm{~mW} / \mathrm{mA}$, which are close to the $0.544 \mathrm{~mW} / \mathrm{mA}$ measured with this setup. Plotting power readings versus current monitor readings or QCL voltage readings do not show similar slopes. We believe that using the additional information of the order in which data points are collected helps average some of the random variations away to show most of the drift in the power readings at the two highest levels is due to increases in the current through the QCL. Subtracting from the power readings for these two power levels, the predicted drift based on linear fits to the current monitor readings or QCL voltage readings and the predicted ratios of $0.544 \mathrm{~mW} / \mathrm{mA}$ and $103 \mathrm{~mW} / \mathrm{V}$ respectively reduces the standard deviations to $5.3 \mu \mathrm{W}$ and $6.3 \mu \mathrm{W}$ or $5.1 \mu \mathrm{W}$ and $6.2 \mu \mathrm{W}$ for the two higher power levels. This still leaves twice the relative standard deviations being about $0.044 \%$. The larger variation may be due to some effect of the collimating lens, such as feedback because of reflections from the lens. However, it might also be due to the higher gain in the QC6N, which makes noise on the control voltage produce twice the current variations as with the QC5. We do not have data that will let us determine the cause of the higher noise level on the collimated Argos QCL system.

Table 3.5. Power Stability for the Collimated Argos QCL for 14.6 Days

\begin{tabular}{lccccc}
\hline & $\begin{array}{c}\mathbf{6} \mathbf{~ m W} \\
\text { Level }\end{array}$ & $\begin{array}{c}\mathbf{1 2} \mathbf{~ m W} \\
\text { Level }\end{array}$ & $\begin{array}{c}\mathbf{1 7} \mathbf{~ m W} \\
\text { Level }\end{array}$ & $\begin{array}{c}\mathbf{2 3} \mathbf{~ m W} \\
\text { Level }\end{array}$ & $\begin{array}{c}\mathbf{2 9} \mathbf{~ m W} \\
\text { Level }\end{array}$ \\
\hline Average Power $(\mathrm{mW})$ & 5.905 & 11.666 & 17.646 & 23.188 & 28.821 \\
Std. Dev. $(\mu \mathrm{W})$ & 4.0 & 4.9 & 4.8 & 7.0 & 8.2 \\
$2 \times$ Rel. Std. Dev. & $0.13 \%$ & $0.084 \%$ & $0.055 \%$ & $0.060 \%$ & $0.057 \%$ \\
\hline
\end{tabular}

\subsubsection{Autopsy of Argos QCL Death}

Unfortunately, measurements on the collimated Argos QCL were terminated by a "buffer error" with the NI-6221 that appears to have resulted in too much current being sent to the Argos QCL and fatally damaging it. Because these measurements used a current supply that provided a maximum current of 2 A, the current limit on the QC6N should have been set to $500 \mathrm{~mA}$ because some of the Argos devices from the same wafer showed catastrophic damage if the current exceeded $500 \mathrm{~mA}$. This safety feature, which is an option on the current controller, would have prevented this catastrophic damage and will be used on future devices if needed to prevent a similar incident. 


\subsection{Burn-In}

In our previous work on this project, both the cryogenic QCL system and the pulsed room temperature system showed the power readings increasing over the months of data collection, a phenomenon we call burn-in. The variability in the data from those systems with their greater sensitivity to laboratory temperatures and other activities in the laboratory, some of which were traced to ground loops interference on the AC power, limited our ability to study burn-in. The greater stability of the custom environmental chamber and the better isolation of the electronics from ground loops and power line fluctuations lead us to a more thorough study of burn-in in QCLs.

Figure 3.8 shows the first power stability data from the Argos QCL, which was taken with cw currents in 8 steps that were 15-seconds long and this cycle was repeated every 10 minutes. This data is the first operation of this QCL except for the time the vendor operated this QCL for testing and a few minutes of operation to determine the current settings to achieve the desired power levels. After a day of operation, the temperature of the QCL was increased from $17.0^{\circ} \mathrm{C}$ to $17.1^{\circ} \mathrm{C}$ to test if temperature fluctuations might be a factor in the observed noise. Table 3.6 lists the burn-in slopes from this data and shows that after increasing the temperature by $0.1^{\circ} \mathrm{C}$, the burn-in slope dropped by a factor of 3 or more for all power levels. The temperature for the next data collection was $20.0^{\circ} \mathrm{C}$ with the same pattern of current steps but the values were adjusted to have about the same power levels as the first run. The burnin slopes were similar to those seen at $17.1^{\circ} \mathrm{C}, 10 \mu \mathrm{W} /$ day for the lower three power levels and about $15 \mu \mathrm{W} /$ day for the high two power levels. This result is quite curious because, for this data set, the cycle was repeated every 123 seconds rather than every 10 minutes. Perhaps the effects of increasing the temperature and decreasing the cycle time canceled each other out. We were focused on the large shortterm fluctuations, which were eliminated by using rapid current modulation, and did not focus on burn-in for several months. The next power stability data we collected was using the $40-\mathrm{kHz}$ current modulation with the standard pattern of steps and was after a total of about 72 hours of current flowing through the QCL. This first current-modulated data gave burn-in slopes of 6-11 $\mu \mathrm{W} / \mathrm{day}$. It was not until the two months of data collected in early 2008 that the experimental setup was unchanged long enough to see possible continuing burn-in. Those burn-in slopes are below $0.6 \mu \mathrm{W} /$ day and are given in Table 3.1.

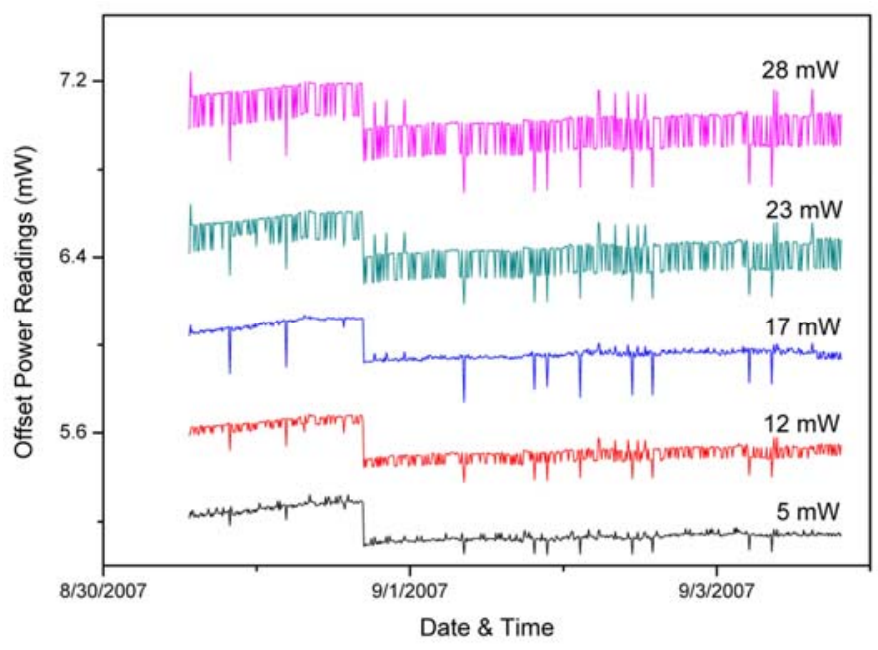

Figure 3.8. First Power Stability Data on Argos QCL 
Table 3.6. Burn-in Slopes from the Initial Data Run for the Argos QCL at Two Slightly Different Temperatures

\begin{tabular}{lccccc}
\hline Power Level $(\mathrm{mW})$ & $5 \mathrm{~mW}$ & $12 \mathrm{~mW}$ & $17 \mathrm{~mW}$ & $23 \mathrm{~mW}$ & $28 \mathrm{~mW}$ \\
Burn-in Slope at $17.0^{\circ} \mathrm{C}(\mu \mathrm{W} /$ day $)$ & 66 & 53 & 68 & 45 & 48 \\
Burn-in Slope at $17.1^{\circ} \mathrm{C}(\mu \mathrm{W} /$ day $)$ & 12 & 16 & 13 & 17 & 17 \\
\hline
\end{tabular}

\subsubsection{Maxion M577G and M577H}

Work concentrating on understanding burn-in began with the pair of FP-QCLs from Maxion identified as M577G and M577H. These QCLs were fabricated from the same wafer and lase at $5.4 \mu \mathrm{m}$ and have high-reflectivity coatings on their back facets. The lasers are AuSn-soldered epi-side down onto an AlN sub-mount and then $\mathrm{Pb} / \mathrm{Sn}$ soldered onto a C-mount. At $20^{\circ} \mathrm{C}$ and with the standard current modulation, the thresholds for these QCLs are $630 \mathrm{~mA}$ and $765 \mathrm{~mA}$ peak currents and the slope efficiency for both are $0.278 \mathrm{~mW} / \mathrm{mA}$. The differential resistances in the operating ranges are $2.42 \mathrm{ohms}$ and 2.04 ohms for M577G and M577H, respectively. The burn-in slopes for the first 24 hours of operation for M577G are given in Table 3.7 and the slopes for the last day of the initial 4 days of operation are given in Table 3.8. After 4 days of operation, the current was set to the $29-\mathrm{mW}$ level, and the cycle time was reduced from 600 seconds to 135 seconds with the current off for 30 seconds to see if this would complete the burn-in process sooner. The burn-in average slope for the initial 24 hours of this new cycle was $62 \mu \mathrm{W} /$ day; about 4 times larger than the preceding slope with the 10-minute cycle, which is in fair agreement with the factor of 4.4 increase in duty cycle. This agreement is consistent with the idea that burn-in is a function of time current flows through the QCL rather than elapsed time.

Table 3.7. Initial Burn-in Slopes for the First Day of Operation

\begin{tabular}{lcrrrr}
\hline Power Level $(\mathrm{mW})$ & $6 \mathrm{~mW}$ & $12 \mathrm{~mW}$ & $18 \mathrm{~mW}$ & $24 \mathrm{~mW}$ & $29 \mathrm{~mW}$ \\
M577G initial burn-in slope $(\mu \mathrm{W} /$ day) & 41 & 46 & 47 & 49 & 51 \\
M577H initial burn-in slope $(\mu \mathrm{W} /$ day) & 96 & 108 & 115 & 130 & 132 \\
M664O initial burn-in slope $(\mu \mathrm{W} /$ day) & 92 & 95 & 99 & 107 & 109 \\
M664M initial burn-in slope $(\mu \mathrm{W} /$ day) & 29 & 24 & 27 & 27 & 25 \\
\hline
\end{tabular}

Table 3.8. Burn-in Slopes for the 5th Day of Operation

\begin{tabular}{lccccc}
\hline Power Level $(\mathrm{mW})$ & $6 \mathrm{~mW}$ & $12 \mathrm{~mW}$ & $18 \mathrm{~mW}$ & $24 \mathrm{~mW}$ & $29 \mathrm{~mW}$ \\
M577G initial burn-in slope $(\mu \mathrm{W} /$ day $)$ & 10 & 12 & 16 & 15 & 17 \\
M577H initial burn-in slope $(\mu \mathrm{W} /$ day) & 7 & 10 & 10 & 11 & 11 \\
M664O initial burn-in slope $(\mu \mathrm{W} /$ day) & 16 & 17 & 17 & 18 & 19 \\
M664M initial burn-in slope $(\mu \mathrm{W} /$ day) & 7 & 6 & 6 & 7 & 5 \\
\hline
\end{tabular}

Figure 3.9 shows the M577G burn-in data as a function of time current was flowing through this QCL as well as the peak current readings and the QCL compliance voltage for the 29-mW power level. Sections of the data corresponding to changes in the measurement duty cycle or configuration, such as 
inserting or removing the Agilent 61/2-digit DMM for measuring the current, have been offset to give continuity. Such changes are accompanied by spikes in the data. The $0.07-\mathrm{mW}$ steep power increase at about 150 hours occurred when the data collection program completed one set of measurements and started the next, with no known change in the setup. In contrast to the Argos QCL, which had largely completed with burn-in after about 80 hours of operation above threshold, this QCL was still changing after 300 hours of operation. Figure 3.10 shows the initial and final four days of data from M577G and Table 3.9 shows the increases in power, current, and QCL voltage readings between the fourth day of operation and the last four days of data. Skipping the first three days of data avoids the most rapidly changing data. The current monitor increase for all power levels is about $40 \mu \mathrm{A}$, which corresponds to a power increase of $0.01 \mathrm{~mW}$. The QCL voltage increase is about $2 \mathrm{mV}$ for all levels, which would correspond to a $0.23-\mathrm{mW}$ power increase if the voltage change were due to a current change. The observed power increases range from $0.28 \mathrm{~mW}$ to $0.39 \mathrm{~mW}$ from low- to high-power levels. The similarity of these power changes to those predicted from the QCL voltage change raises the question of whether the higher currents required for this QCL are affecting the stability of the QC5 and also leading to errors in the current monitor output. If that were the case, however, would the currents still be increasing after almost a month of nearly continuous operation? The fact that the power increase is nearly independent of power level is strong evidence that the power increase is not due to changes in the power meter. With zero subtraction for each cycle, long-term power meter drifts are subtracted out leaving changes in the proportionality constant between incident power and recorded signal as the other obvious class of possible changes. Such changes would give power reading changes that increase proportionally with the power level, which is clearly not the case for this QCL.

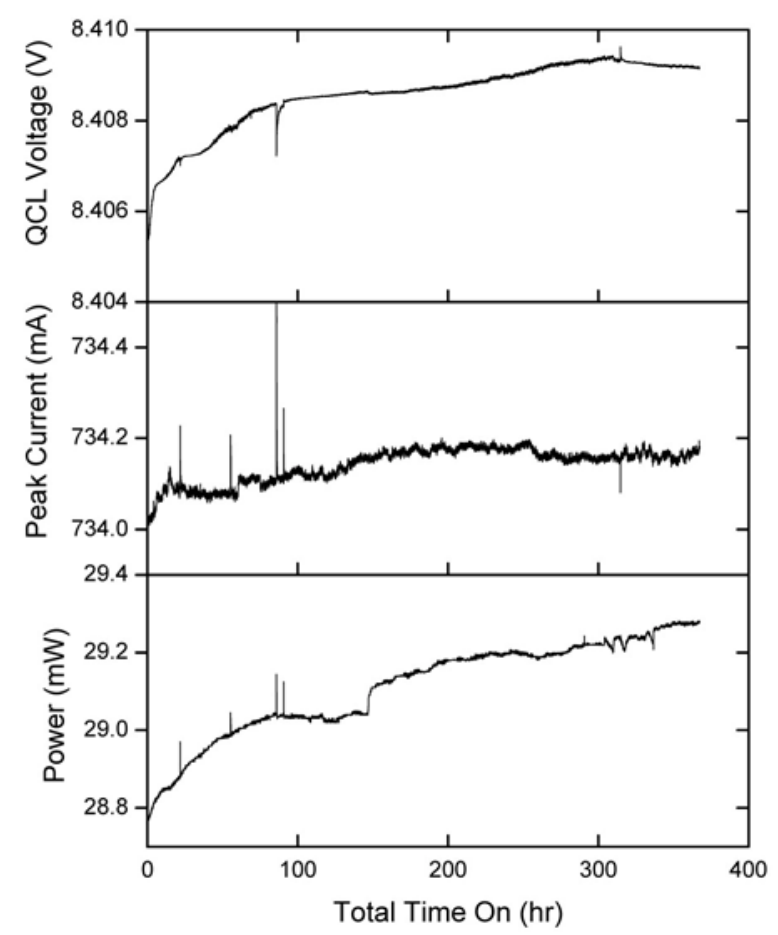

Figure 3.9. Burn-in of Power and Measured Changes in Current Readings and Compliance Voltage at the 29-mW Power Level for M577G 


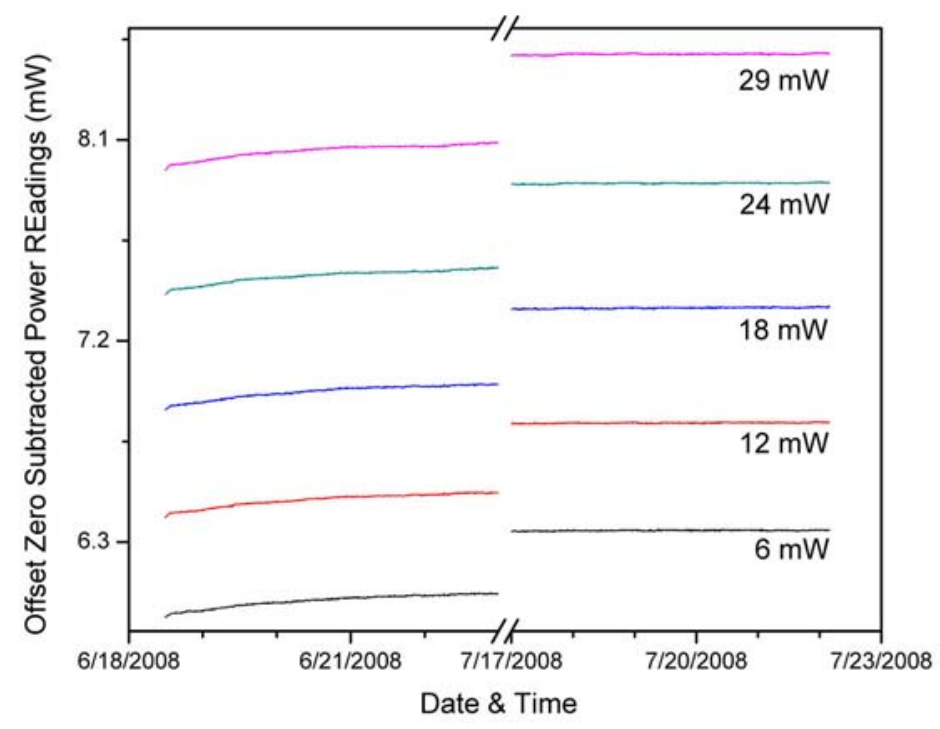

Figure 3.10. Initial and Final 5 Days of Burn-in for M577G

Table 3.9. Changes in Power Readings, Current Monitor Readings, and QCL Voltage Readings Between the Fifth Day of Data Collection and the Last Four Days of Data Collection on M577G

\begin{tabular}{lccccc}
\hline Power Level $(\mathrm{mW})$ & $6 \mathrm{~mW}$ & $12 \mathrm{~mW}$ & $18 \mathrm{~mW}$ & $24 \mathrm{~mW}$ & $29 \mathrm{~mW}$ \\
Power Increase $(\mathrm{mW})$ & 0.281 & 0.310 & 0.338 & 0.376 & 0.393 \\
Peak Current Increase $(\mu \mathrm{A})$ & 40.6 & 38.7 & 38.6 & 39.1 & 38.6 \\
QCL Voltage Increase $(\mathrm{mV})$ & 1.92 & 1.68 & 1.94 & 1.96 & 2.01 \\
\hline
\end{tabular}

Table 3.7 shows the burn-in slopes for M577H for the initial 24 hours, which are more than twice the values for M577G. Table 3.8 shows that by the fifth day of operation with the normal measurement cycle these slopes are significantly smaller than those for M577G. These slopes increased in the next 16 hours to values larger than those of M577G on the fifth day. Similar to M577G, after about 51/2 days of initial data collection, we changed to a 135-second cycle with the QCL at $29 \mathrm{~mW}$ for 90 of those seconds and just below threshold for 15 additional seconds, the "burn-in cycle.” The average burn-in slope is about $60 \mu \mathrm{W} /$ day for the first day of this accelerated burn-in, which is about six times the burn-in slope before changing the cycle. This increase is 35\% more than the factor of 4.4 increase expected from the increased duty cycle, but the slopes can vary by about a factor of 2 from day to day.

Figure 3.11 shows the changes in power, current, and QCL voltage readings burn-in measurements on $\mathrm{M} 577 \mathrm{H}$ for the $29 \mathrm{~mW}$ power level. The power readings for the approximately 250 hours when using the "burn-in cycle" were offset to make the power readings continuous. The corresponding current monitor readings have not been adjusted and show a slight reduction in current when using the burn-in cycle. This current drop is probably due to the increased heating in the current supply. After switching back to using the standard cycle, the power readings decreased slightly for about 2 weeks at all power levels, as can be seen in Figure 3.12. Table 3.10 lists the changes in power, current and QCL voltage readings between the last day of the initial burn-in using the standard cycle and the last 4 days of data. Using the $0.278 \mathrm{~mW} / \mathrm{mA}$ slope efficiency of this QCL, the increase in current monitor readings of $0.06 \mathrm{~mA}$ only 


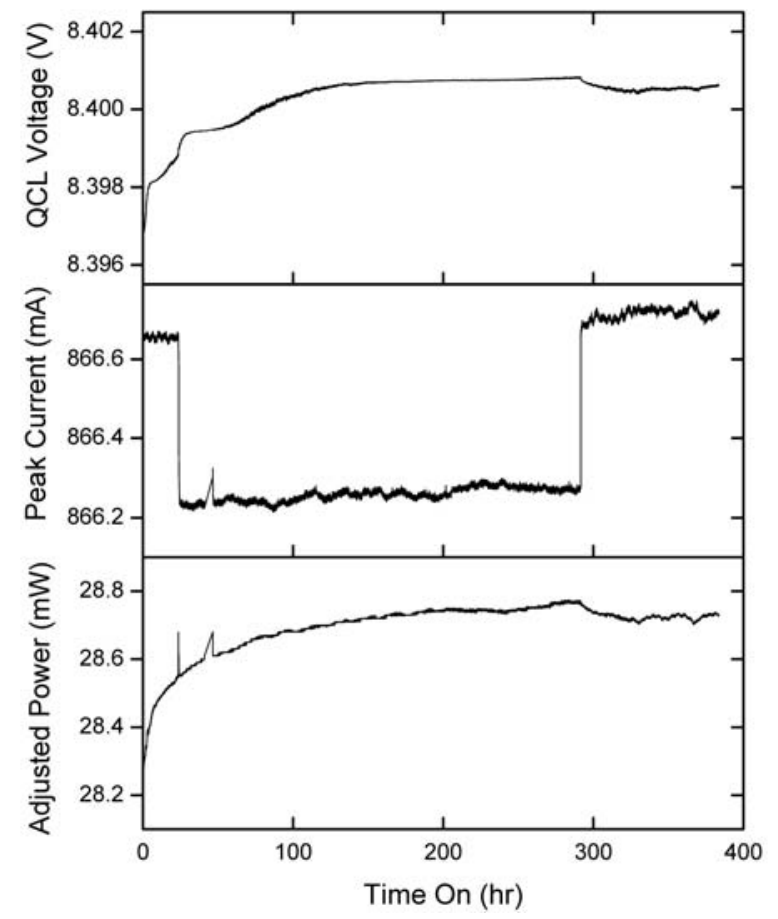

Figure 3.11. Burn-in of Power and Measured Changes in Current Readings and Compliance Voltage at the 29-mW Power Level for M577H

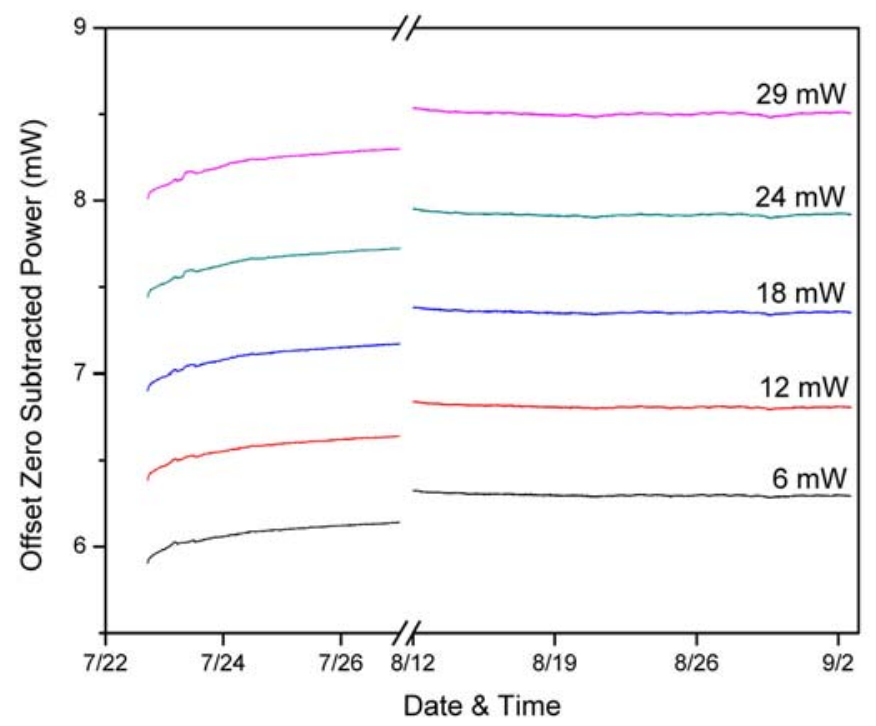

Figure 3.12. Initial Power Readings and Readings After the Approximately 250 Hours of the Burn-in Cycle for M577H 
Table 3.10. Changes in Power Readings, Current Monitor Readings, and QCL Voltage Readings Between the Last Day of the Standard Cycle Before Starting to Use the Burn-in Cycle and the Last Four Days of Data Collection on M577H

\begin{tabular}{lccccc}
\hline Power Level $(\mathrm{mW})$ & $6 \mathrm{~mW}$ & $12 \mathrm{~mW}$ & $18 \mathrm{~mW}$ & $24 \mathrm{~mW}$ & $29 \mathrm{~mW}$ \\
Power Increase $(\mathrm{mW})$ & 0.144 & 0.155 & 0.169 & 0.181 & 0.187 \\
Peak Current Increase $(\mu \mathrm{A})$ & 60 & 60 & 59 & 58 & 58 \\
QCL Voltage Increase $(\mathrm{mV})$ & 1.5 & 1.7 & 1.6 & 1.6 & 1.9 \\
\hline
\end{tabular}

predicts a $0.017 \mathrm{~mW}$ power increase; about $10 \%$ of the observed increase. In contrast, the QCL voltage increases, assuming that the voltage change is only due to a current change and using the measured differential resistance of 2.04 ohms, predicts power increases between $0.20 \mathrm{~mW}$ and $0.26 \mathrm{~mW}$. This rough agreement between these predictions and the observed power reading changes again raises the question if changes in current that are not accurately reflected by the current monitor readings could be responsible for the observed power increases. As for M577G, the power increases for M577H are nearly independent of power level, which removes the power measurement from being a candidate for the source of these power increases.

\subsubsection{Maxion M664L, M664M, and M6640}

After discussions with both John Bruno at Maxion and Claire Gmachl at Princeton University, we believed the burn-in could be due to improved thermal contact between the laser and the sub-mount resulting from the solder softening from the high temperatures reaching this interface as current flows through the QCL. As the solder softens, any stresses present at this thermal interface could be relieved and the thermal performance improved. If this hypothesis were true, thermally annealing the devices prior to operation should affect the burn-in behavior. Thus, we used three new QCLs from the same wafer to explore ways to reduce, speed up, or eliminate the burn-in effect in new QCLs. These are FabryPerot QCLs with high-reflection coatings on the back facets that are mounted “epi-down” onto an AlN sub-mount soldered onto a modified C-mount. To see if annealing would influence the burn-in, these three QCLs were annealed in air at $150^{\circ} \mathrm{C}$. After six days $\mathrm{M} 664 \mathrm{O}$ was removed from the oven for testing, while the other two QCLs remained in the oven. After 11 days at $150^{\circ} \mathrm{C}$, the oven temperature was increased to $165^{\circ} \mathrm{C}$ for two days.

\subsubsection{M6640}

Measurements on $\mathrm{M} 664 \mathrm{O}$, after six days at $150^{\circ} \mathrm{C}$, began with almost six days of operation with the standard cycle. The system was very stable for this run with the standard deviation of the difference between the second and last cycle of $1.6 \mu \mathrm{W}$. The threshold was $730 \mathrm{~mA}$ of peak current, the slope efficiency was $0.224 \mathrm{~mW} / \mathrm{mA}$, and the differential resistance was 1.83 ohms. The burn-in slopes from the first and fifth day are listed in Table 3.6 and Table 3.7 and are quite similar to the analogous slopes for M577G and M577H. There appears to be no substantial change in burn-in behavior due to six days of annealing at $150^{\circ} \mathrm{C}$.

Based on a suggestion from Claire Gmachl at Princeton University, we tried to accelerate the burn-in by using a cw current and a duty cycle near 100\%. Specifically, the first two steps are 15-seconds long and just below threshold, while the next five steps are at the same current setting and targeted to produce 
$29.5 \mathrm{~mW}$. The last step is 30-seconds long and with the current off. The cycle immediately repeats after the end of the last step. The fraction of time during a cycle that current flows through the QCL with this cycle is $95.6 \%$, while for the standard cycle current is flowing $8.75 \%$ of the time including the $50 \%$ duty cycle of the current modulation. With the previous burn-in cycle, this fraction is $43.75 \%$. M664O was operated with this cw burn-in cycle for 47.4 hours during which time the burn-in slope changed from $440 \mu \mathrm{W} /$ day to $360 \mu \mathrm{W} /$ day. The burn-in slope for this power level using the standard cycle is $19 \mu \mathrm{W} /$ day, which scales to the same fraction of time current is flowing equivalent to $208 \mu \mathrm{W} /$ day. There appears to be an additional factor of two increase in burn-in rate with this cw burn-in cycle than can be explained by the increase in the fraction current is flowing. The average current is only $15 \%$ larger with the cw burn-in cycle, so increased peak current is not likely to be a significant contribution. Figure 3.13 shows the power readings for M664O using the standard cycle after this accelerated burn-in. Initially there is an increase in the burn-in slopes to about $45 \mu \mathrm{W} /$ day, but these slopes decrease to about $6 \mu \mathrm{W} /$ day for the last 24 hours of data.

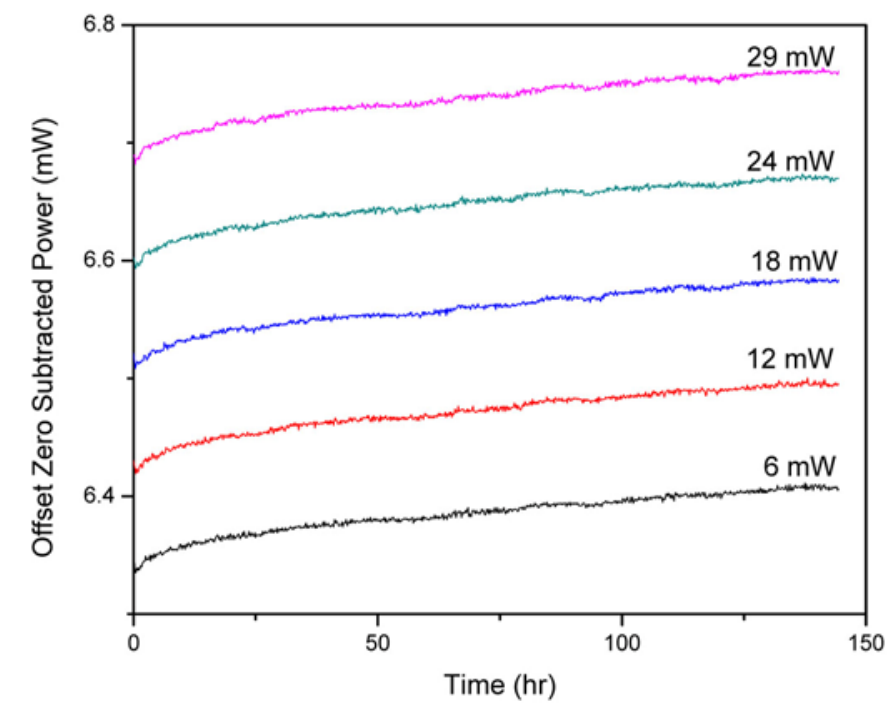

Figure 3.13. M664O Power Readings Using Standard Cycle Following 47.4 Hours of the cw Burn-in Cycle

Table 3.11 shows the changes in power, current, and QCL voltage readings for M664O from the 24 hours before the start of the cw burn-in cycle and the last 24 hours of the data in Figure 3.13. The current monitor changes predict power changes of $0.022 \mathrm{~mW}$ while using the 1.83-ohm differential resistance and the QCL voltage changes predicts power changes of $0.73 \mathrm{~mW}$. This result argues against the power change being due to current changes that are not reflected in the current monitor readings. 
Table 3.11. Changes in Power Readings, Current Monitor Readings, and QCL Voltage Readings Between the Last Day of the Standard Cycle Before Starting to Use the cw Burn-in Cylce for 47.4 Hours and the Last Day of Data Collection on M664O

\begin{tabular}{lccccc}
\hline Power Level $(\mathrm{mW})$ & $6 \mathrm{~mW}$ & $12 \mathrm{~mW}$ & $18 \mathrm{~mW}$ & $24 \mathrm{~mW}$ & $29 \mathrm{~mW}$ \\
Power Increase $(\mathrm{mW})$ & 0.201 & 0.210 & 0.217 & 0.227 & 0.233 \\
Peak Current Increase $(\mu \mathrm{A})$ & 10 & 9 & 11 & 10 & 9 \\
QCL Voltage Increase $(\mathrm{mV})$ & 5.9 & 6.5 & 6.4 & 5.9 & 6.2 \\
\hline
\end{tabular}

\subsubsection{M664M}

The QCL M664M was annealed at $150^{\circ} \mathrm{C}$ for 11 days and then at $165^{\circ} \mathrm{C}$ for 2 days before we mounted it in the test setup. Given the lack of significant improvement in the burn-in behavior of M664O with annealing, we tried operating M664M at a continuous-wave current of $500 \mathrm{~mA}$ at an elevated temperature, $50^{\circ} \mathrm{C}$, to try to accelerate the burn-in. This QCL was operated this way for 5 days with the current turned off for 30 seconds every 20 minutes. After this pre-burn-in, we operated M664M with the standard cycle for over 18 days, with the power data shown in Figure 3.14. The threshold was $652 \mathrm{~mA}$, the slope efficiency $0.202 \mathrm{~mW} / \mathrm{mA}$, and the differential resistance was $2.03 \mathrm{ohms}$. The initial burn-in slopes are listed in Table 3.7 and are about $27 \mu \mathrm{W} /$ day, which is significantly smaller than the initial burn-in slopes in that table. However, M664M was operated for five days below threshold, so perhaps it is more meaningful to compare these slopes with those for the fifth day of operation of M664O, which are about 30\% lower. The slopes for M664M on the fifth day of operation with the standard cycle are listed in Table 3.8 and they are three times smaller than those for M664O in the same table. Over the last week of data in Figure 3.14, the burn-in slopes are about $4 \mu \mathrm{W} /$ day. It is not clear that the combination of the annealing and the sub-threshold burn-in at $50^{\circ} \mathrm{C}$ have substantially improved burn-in other than doing the rapid initial burn-in with below-threshold current so it is not measured.

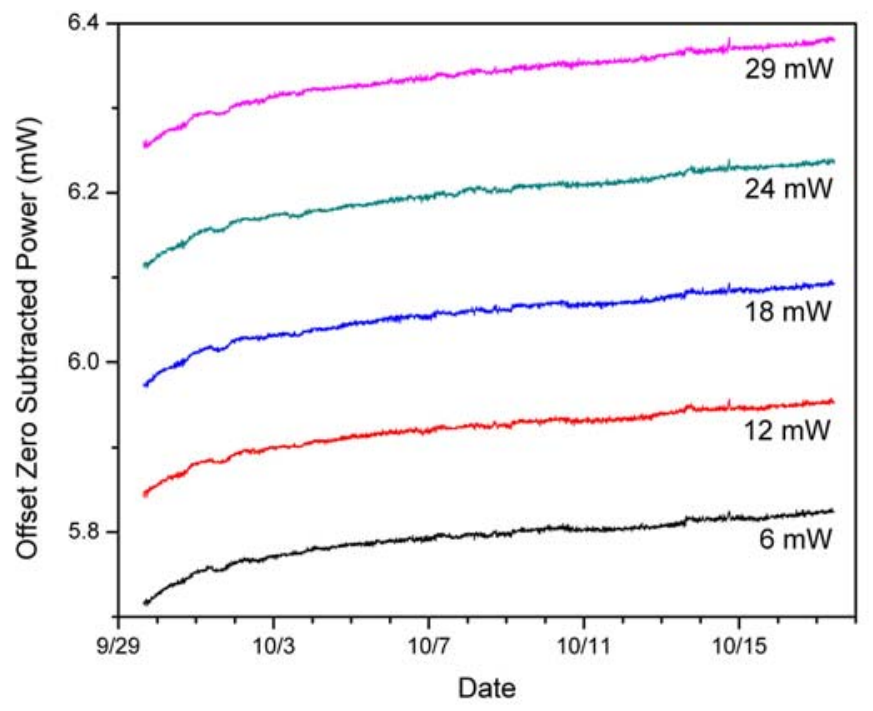

Figure 3.14. Power Stability Measurements of M664M Using the Standard Cycle After the cw Subthreshold Operation at $50^{\circ} \mathrm{C}$ 
Because the burn-in showed no sign of stopping quickly, we operated M664M at the maximum current of the QC5 current controller, $939 \mathrm{~mA}$, for five days. The current was not modulated and every 20 minutes it was set to zero to allow measuring the power meter zero. Figure 3.15 shows the zerosubtracted power readings during this burn-in. Most striking is the approximately quantized levels of the zero-subtracted power readings, which reflect the $0.1-\mathrm{mW}$ output quantization of the 3 Sigma $^{\mathrm{TM}}$ power meter on the $300-\mathrm{mW}$ scale. The averaging of readings of both the zero and power readings is responsible for values not being exact multiples of $0.1 \mathrm{~mW}$. The average burn-in slope is $85 \mu \mathrm{W} / \mathrm{day}$, which is twice the slope from the last week of data in Figure 3.15 scaled by the inverse of the $8.75 \%$ duty cycle of the standard cycle. After operating at $939 \mathrm{~mA}$ and returning to the standard cycle, the burn-in slopes were still about $4 \mu \mathrm{W} /$ day, decreasing to about $2.5 \mu \mathrm{W} /$ day for the last two days of a week with the standard cycle. Table 3.12 lists the changes in power, current monitor reading, and QCL voltage due to running at $939 \mathrm{~mA}$. The current monitor readings actually decreased while the power and QCL voltage readings increased. The QCL voltage changes in conjunction with the differential resistance and the slope efficiency predict power changes that are about three times those observed, which supports the theory that these power changes are not caused by changes in the current through the QCL.

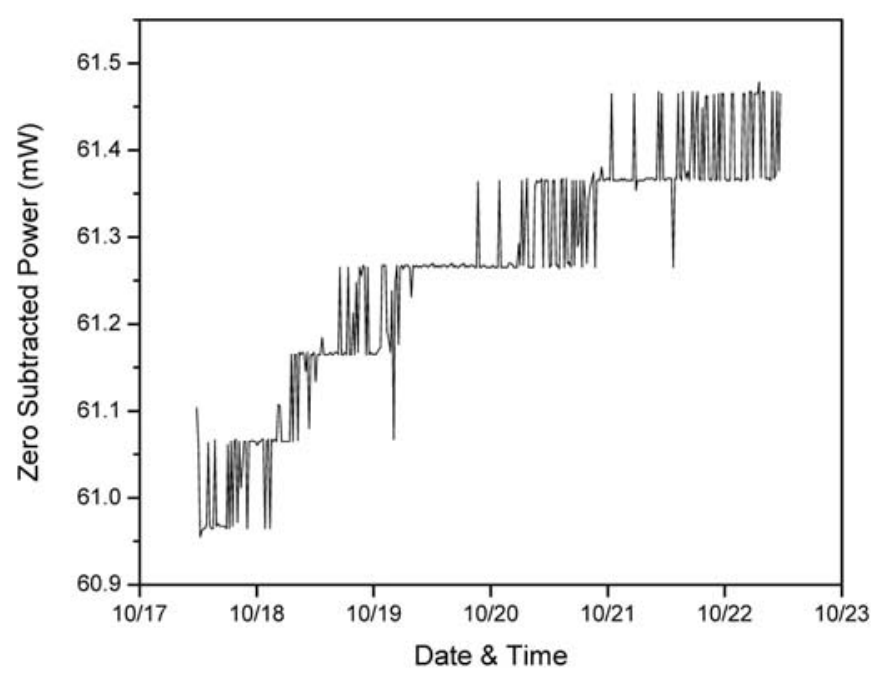

Figure 3.15. Power During Burn-in of M664M at $939 \mathrm{~mA}$

Table 3.12. Changes in Power Readings, Current Monitor Readings, and QCL Voltage Readings Between the Last Day of the Standard Cycle Before the 939 mA Burn-in and the First Day of the Standard Cycle Measurements Afterwards

\begin{tabular}{lccccc}
\hline Power Level $(\mathrm{mW})$ & $6 \mathrm{~mW}$ & $12 \mathrm{~mW}$ & $18 \mathrm{~mW}$ & $24 \mathrm{~mW}$ & $29 \mathrm{~mW}$ \\
Power Increase $(\mathrm{mW})$ & 0.140 & 0.152 & 0.158 & 0.170 & 0.179 \\
Peak Current Increase $(\mu \mathrm{A})$ & -59 & -58 & -58 & -58 & -57 \\
QCL Voltage Increase $(\mathrm{mV})$ & 4.4 & 4.3 & 4.9 & 4.9 & 4.3 \\
\hline
\end{tabular}

To further explore the effect of higher current operation on the power stability of the QCL, we operated M664M at $1200 \mathrm{~mA}$ for five days with the same pattern as we used for the $939 \mathrm{~mA}$ burn in. To achieve this higher current, we used a PNNL-made current controller capable of -2 ampere output, model 
number QC6N. This higher current was determined following advice from Maxion to increase the current until the slope of the power-versus-current curve decreased from its value at threshold by a factor of two. This approach has the advantage of operating the QCL continuously at higher current without potentially stressing the QC5 that is used in making the stability measurements. Table 3.13 lists the changes due to this burn-in. The power increase is about half that observed with the 939-mA burn-in as are the QCL voltage changes. Once the standard cycle was resumed while again using the QC5, the burn-in slopes were increased to $16 \mu \mathrm{W} /$ day for the first 56 hours and then they decreased to between 4 and $9 \mu \mathrm{W} /$ day as can be seen in Figure 3.16.

Table 3.13. Changes in Power Readings, Current Monitor Readings, and QCL Voltage Readings Between the Last Day of the Standard Cycle Before the 1200-mA Burn-in and the First Day of the Standard Cycle Measurements Afterwards

\begin{tabular}{lccccc}
\hline Power Level $(\mathrm{mW})$ & $6 \mathrm{~mW}$ & $12 \mathrm{~mW}$ & $18 \mathrm{~mW}$ & $24 \mathrm{~mW}$ & $29 \mathrm{~mW}$ \\
Power Increase $(\mathrm{mW})$ & 0.066 & 0.070 & 0.076 & 0.078 & 0.084 \\
Peak Current Increase $(\mu \mathrm{A})$ & 5.4 & 8.6 & 11.4 & 14.9 & 15.9 \\
QCL Voltage Increase $(\mathrm{mV})$ & 2.3 & 2.4 & 2.4 & 2.4 & 2.6 \\
\hline
\end{tabular}

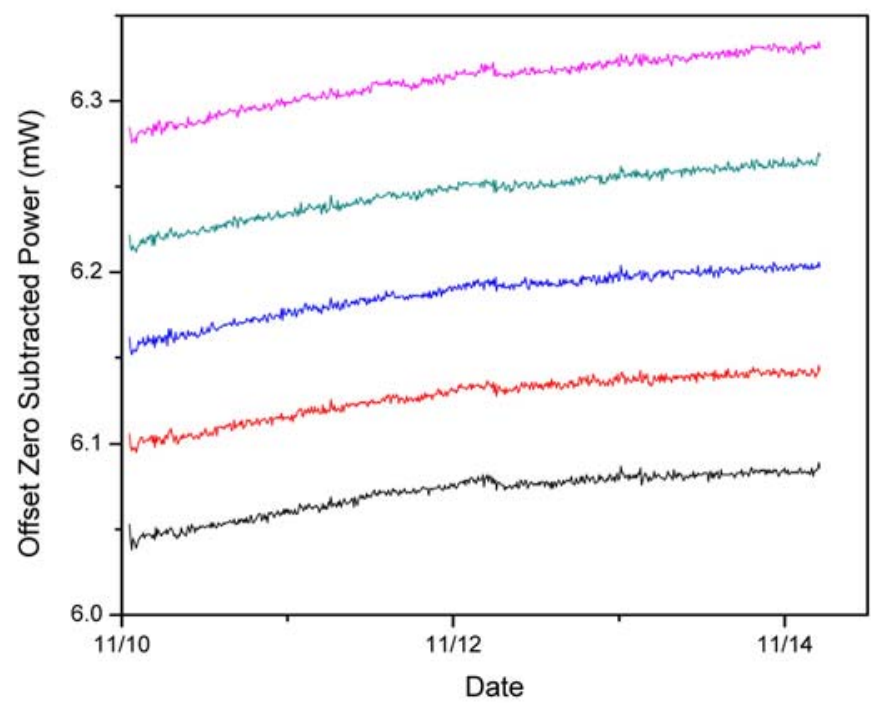

Figure 3.16. Power Readings with Standard Cycle for M664 M After 1200-mA Burn in for 5 Days

\subsubsection{Maxion M575D DFB}

The first room temperature, continuous-wave QCL that has a distributed feedback grating (DFB) that we received was the Maxion QCL M575D. Its lasing wavelength is $5.36 \mu \mathrm{m}$ near room temperature. At $20^{\circ} \mathrm{C}$, the threshold is $675 \mathrm{~mA}$ and the slope efficiency is $0.559 \mathrm{~mW} / \mathrm{mA}$. The QCL is soldered "epi-side down" to an AlN sub-mount, which matches the coefficient of thermal expansion of the QCL, which is soldered onto a modified C-mount. There is a high reflectivity coating on the rear facet and an antireflection coating on the output facet. To assess the power stability of M575D and look for burn-in, we operated this QCL with continuous-wave currents in a cycle with eight 15-second-long steps. The first 
two steps were intended to be below threshold, but the threshold was clearly changing for the first few hours of operation. The next five steps were at currents giving power levels near $6,12,18$, 24, and $29 \mathrm{~mW}$, while the final step was with the current at zero. Figure 3.17 shows the power readings during the initial 5 days of operation of M575D. The current level for the second step was below threshold at the beginning of data collection, but as can be seen from the " $0 \mathrm{~mW}$ " trace, the threshold moved below this current level and then back above it during the first several hours. The power readings at the other power levels also showed large changes, but the current monitor readings show no evidence of corresponding drifts to within the $12 \mu \mathrm{A}$ standard deviations of the readings. After the first day, the powers are all drifting down at about the same rate of $-80 \mu \mathrm{W} /$ day, which is almost 10 times larger in magnitude and of the opposite sign as we have seen for other QCL for the 2-5 days of burn-in. The difference between the first and last step values, when the QCL is not lasing, has a standard deviation of $2.5 \mu \mathrm{W}$. This value is consistent with similar measurements of the variability of the measurement system for similar durations. This confirms that the variability is not due to the power meter. After changing to the standard modulated current cycle, the powers continued to decline but at about half the rate. This change in these rates is consistent with the $50 \%$ duty cycle of the modulated current and suggests that the decline is proportional to the time current is flowing through the QCL.

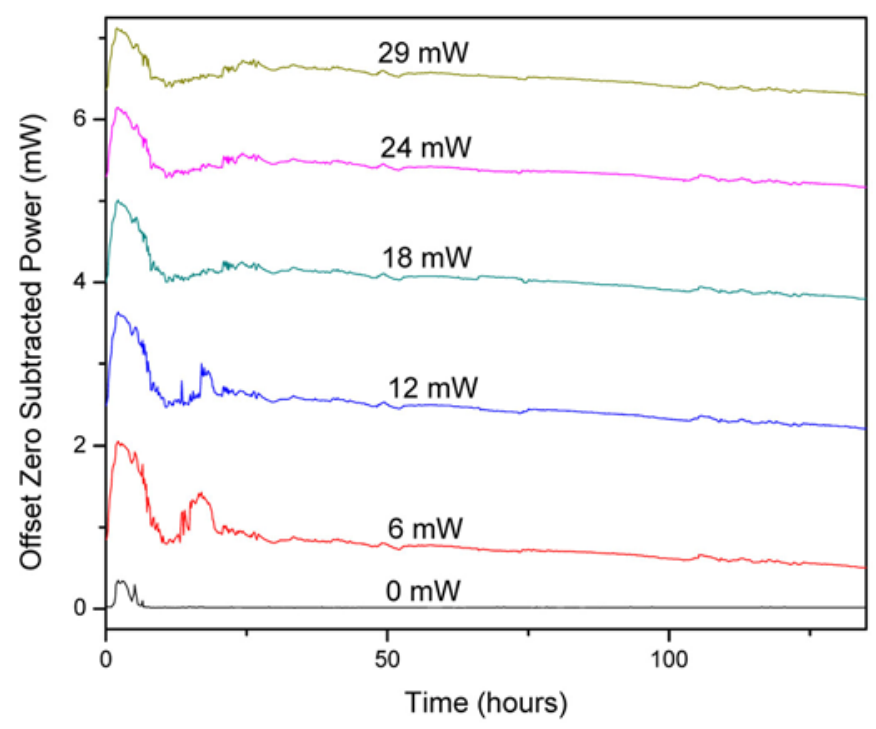

Figure 3.17. Power Readings from M575D During Initial Burn-in. Power readings from the first step are used for the zero subtraction and the trace labeled " $0 \mathrm{~mW}$ " is from the second current step, which initially was below threshold.

After we ended this power stability testing, we measured the spectrum of the uncollimated emission of this QCL with a Fourier transform infrared (FTIR). There were clearly two longitudinal modes lasing except at currents only slightly above threshold. It is interesting that after mounting this QCL in an highheat load (HHL) package and adding a collimating lens, measurements with the same FTIR, a highresolution infrared wave meter, and absorption by atmospheric water all show this QCL lasing on a single frequency. 


\subsubsection{Princeton A887a8}

The laser system that we started testing in FY06 (Myers et al. 2006) continued throughout FY08. This QCL, which is a research device from Princeton University, is indium-soldered epi-up onto a copper C-mount. The laser system is set up on an optical table in the laboratory so that it is more susceptible to temperature fluctuations from the HVAC compared to the system in the custom environmental enclosure. Gray foam encloses the power meter to shield the detector from rapid changes such as personnel moving about the laboratory. The fluctuations on the power meter measurements in this set-up are larger than the variations in the custom environmental enclosure. Nonetheless, this system has provided a crude testbed for measuring the long-term repeatability of a thermoelectrically cooled QCL. A low duty cycle is used to minimize the waste heat from the QCL because no additional cooling is used for the heat sink. The laser is operated at $15^{\circ} \mathrm{C}$ with a $1-\mu$ s pulse width and a $10 \%$ duty cycle. The current is ramped over five radiance levels in which a new cycle is started every 134 seconds providing a 30-second delay between cycles. About 644 cycles are completed every day. Over 400,000 cycles were completed before the system was shut down.

Fairly good performance is observed for this laser over the first 349,600 cycles, which reflects over 10,000 hours of operation. Figure 3.18 shows the change in power observed for each step above threshold. Table 3.14 displays the average power and standard deviation for all five steps above threshold.

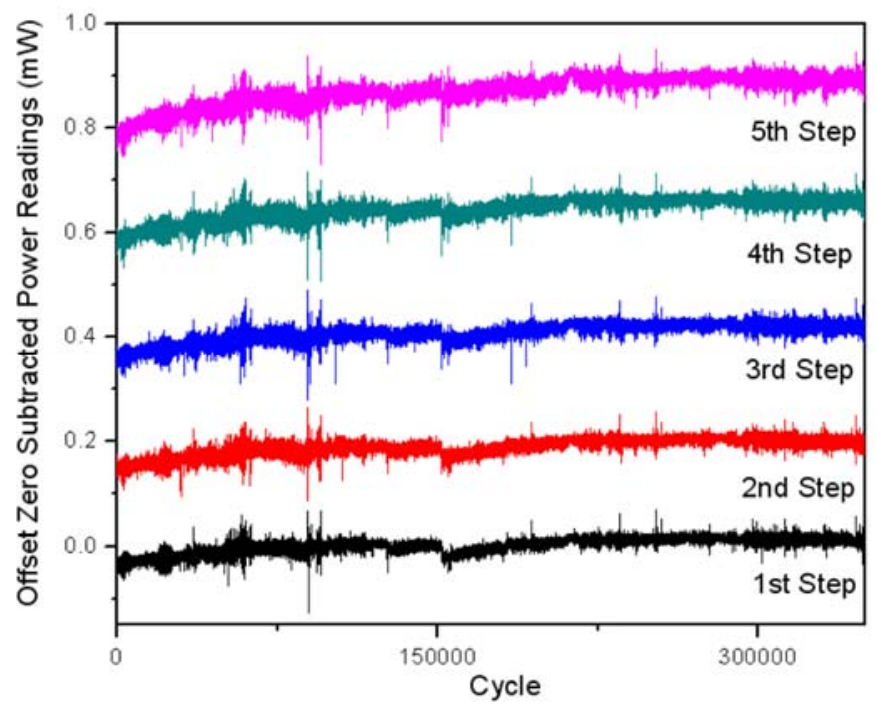

Figure 3.18. Power Readings for A887a8 Over 349600 Cycles or 542 Days of Operation

Table 3.14. Long-Term Power Stability for A8887a8 Over 542 Days of Operation

\begin{tabular}{lccccc}
\hline & $\begin{array}{c}\text { First } \\
\text { Level }\end{array}$ & $\begin{array}{c}\text { Second } \\
\text { Level }\end{array}$ & $\begin{array}{c}\text { Third } \\
\text { Level }\end{array}$ & $\begin{array}{c}\text { Fourth } \\
\text { Level }\end{array}$ & $\begin{array}{c}\text { Fifth } \\
\text { Level }\end{array}$ \\
\hline Average Power $(\mathrm{mW})$ & 0.571 & 1.171 & 1.760 & 3.001 & 5.580 \\
Std. Dev. $(\mu \mathrm{W})$ & 13.8 & 15.4 & 17.1 & 20.6 & 27.7 \\
$2 \times$ Rel. Std. Dev. & $4.5 \%$ & $2.6 \%$ & $1.9 \%$ & $1.3 \%$ & $1.0 \%$ \\
\hline
\end{tabular}


The biggest contribution to the long-term drift is the burn-in or the increase in output power at all power levels as discussed in Section 3.2. The data in Figure 3.19 and also in Table 3.15 shows the average power from the first 5000 cycles and the last 5000 cycles for each step above threshold. The standard deviation over this timeframe varies from 5-7 microwatts as is primarily limited by the poor isolation from laboratory-temperature variations.

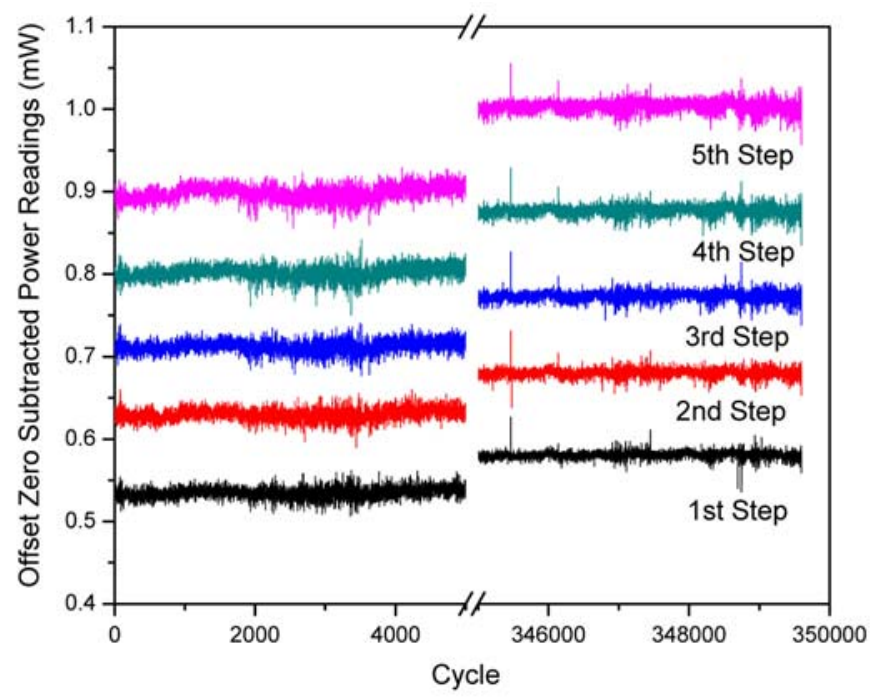

Figure 3.19. Initial and Final Power Readings for A887a8 Illustrating the Increase in Power that is Observed at All Steps above Threshold

Table 3.15. Initial (first 5000 points) and Final (last 5000 points) Average Powers for A887a8

\begin{tabular}{lccccc}
\hline & $\begin{array}{c}\text { First } \\
\text { Level }\end{array}$ & $\begin{array}{c}\text { Second } \\
\text { Level }\end{array}$ & $\begin{array}{c}\text { Third } \\
\text { Level }\end{array}$ & $\begin{array}{c}\text { Fourth } \\
\text { Level }\end{array}$ & $\begin{array}{c}\text { Fifth } \\
\text { Level }\end{array}$ \\
\hline Initial Average Power $(\mathrm{mW})$ & 0.534 & 1.129 & 1.712 & 2.941 & 5.498 \\
Final Average Power $(\mathrm{mW})$ & 0.580 & 1.180 & 1.773 & 3.017 & 5.603 \\
$\Delta$ Power $(\mathrm{mW})$ & 0.046 & 0.051 & 0.061 & 0.076 & 0.105 \\
Percent Increase & $8.5 \%$ & $4.4 \%$ & $3.6 \%$ & $2.6 \%$ & $1.9 \%$ \\
\hline
\end{tabular}

If we ignore the first 150,000 cycles, which accounts for most of the burn-in, we obtain very good results over almost 200,000 cycles for almost 5800 hours of operation. The average power and standard deviation for each step are shown in Table 18. A standard deviation around $10 \mu \mathrm{W}$ is observed for all of the steps. These fluctuations are primarily due to temperature fluctuations affecting the measurement set-up. 
Table 3.16. Power Stability for A887a8 After the Device has been Burned in (includes cycles from 150,000-349600)

\begin{tabular}{lccccc}
\hline & $\begin{array}{c}\text { First } \\
\text { Level }\end{array}$ & $\begin{array}{c}\text { Second } \\
\text { Level }\end{array}$ & $\begin{array}{c}\text { Third } \\
\text { Level }\end{array}$ & $\begin{array}{c}\text { Fourth } \\
\text { Level }\end{array}$ & $\begin{array}{c}\text { Fifth } \\
\text { Level }\end{array}$ \\
\hline Average Power $(\mathrm{mW})$ & 0.579 & 1.179 & 1.769 & 3.013 & 5.598 \\
Standard Deviation $(\mu \mathrm{W})$ & 9.5 & 10.2 & 10.3 & 10.9 & 12.5 \\
$2 \times$ Rel. Std. Dev. & $3.3 \%$ & $1.7 \%$ & $1.2 \%$ & $0.7 \%$ & $0.4 \%$ \\
\hline
\end{tabular}

After the first 349,600 cycles, however, a rapid degradation is observed in the output power as illustrated in Figure 3.20. This degradation does not appear to be the result of deterioration due to the growth of defects in the active region because a slow degradation in the performance of the laser would be expected. This random failure is more likely the result of damage to the output facet because it is exposed to ambient air or degradation of the heat sink or bonds. The random failure resulted in a sudden drop over approximately 47 cycles after which the power begins a slower degradation for another 31,550 cycles or almost 50 days of operation until it settles down and equilibrates. This failure does not appear to be due to a change in the responsivity of the power meter because the loss in power is not proportional to the power before the degradation. Instead, these changes show that both the slope efficiency and the threshold of the device have changed. Table 3.17 shows the slope efficiency and threshold current calculated from a linear fit of the data in Figure 3.20 prior to degradation (344600349600), right after the degradation (350000-353000) and after the power has equilibrated after the degradation (381000-405000).

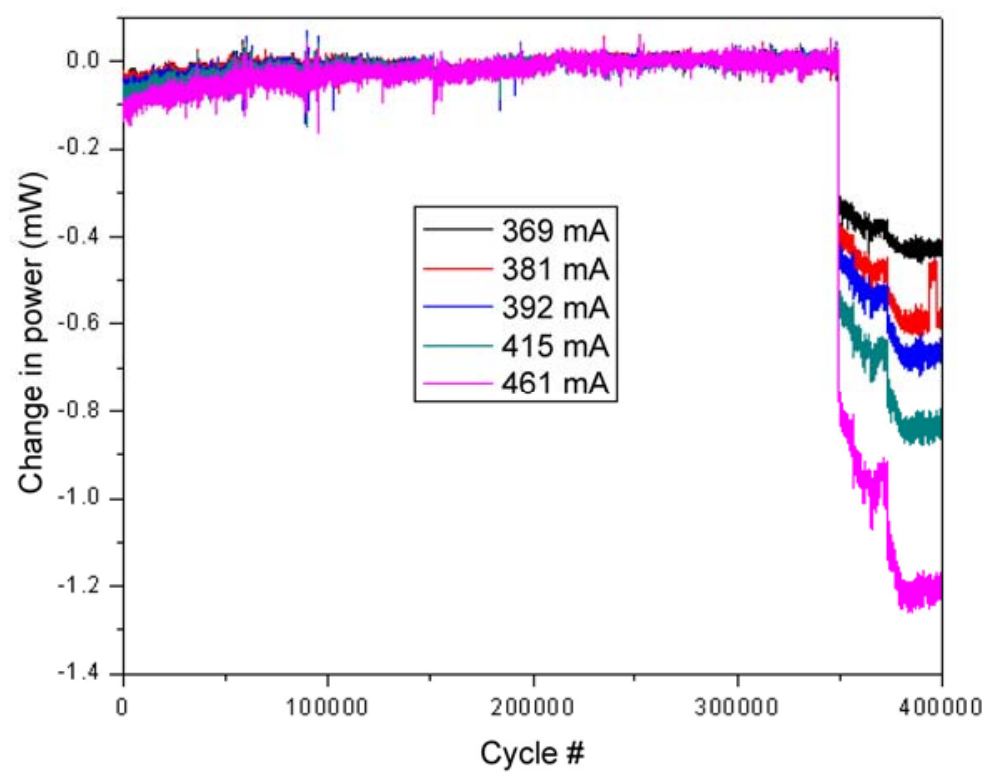

Figure 3.20. Power Readings for A887a8 Over 400000 Cycles Illustrating the Sudden Degradation that was Observed 
Table 3.17. Slope Efficiencies and Threshold Currents for Sections of Data from Figure 3.20

\begin{tabular}{lccc}
\hline Section (cycle) & $344600-349600$ & $350000-353000$ & $381000-405000$ \\
Slope efficiency (mW/mA) & 0.05481 & 0.0496 & 0.0466 \\
Threshold current (mA) & 359.3 & 365.0 & 367.4 \\
$2 \times$ Rel. Std. Dev. & $3.3 \%$ & $1.7 \%$ & $1.2 \%$ \\
\hline
\end{tabular}

These changes in the threshold current and slope efficiency could be due to changes in the thermal properties of the laser assembly so that heat is removed less efficiently from the active region. The laser die attach solder interface can significantly impact the thermal performance and reliability of a laser assembly. In particular, voids in the die attach can degrade thermal performance and limit the lifetime. Because the attachment of the die remains a technical challenge for the manufacturers of QCLs, a suboptimal die attach could be the reason for the sudden degradation. Another possibility could be because this device was soldered using Indium solder. Usually, manufacturers prefer to use gold/tin (AuSn) solder, which is harder and has a higher melting point than indium. Furthermore, studies have shown that the lifetime of lasers using Indium solder is shorter compared to lasers using AuSn solder because of electromigration of the Indium solder, which creates voids and local heating at the facets (Liu et al. 2006). This mechanism is not gradual but results in a sudden degradation and ultimately leads to failure. Better long-term results should be achieved with commercial devices from companies that have achieved good uniformity with their die attach process as well as with hermetically sealed devices to prevent any problems with repeated exposure to moisture in the ambient air and to particles floating in the air that could lead to damage at the facets. Nevertheless, this laser still performed quite well and no degradation was observed for the first 10,000 hours or 542 days of operation.

One other item that should be mentioned is that the power meter was returned to Coherent for recalibration in the first quarter of 2008. Coherent performs the calibration procedure at $514 \mathrm{~nm}$ and claims an uncertainty of $\pm 1 \%$ for the power meter head and an uncertainty of $\pm 3 \%$ for the power meter read-out. The original calibration that occurred in December 2005 measured a voltage responsivity of $0.1481 \mathrm{~V} / \mathrm{W}$. When the system was sent back for re-calibration in April 2008, the voltage responsivity was measured to be $0.1345 \mathrm{~V} / \mathrm{W}$. These values correspond to a $9.2 \%$ change in responsivity. It is unclear if the responsivity changed during the measurement but seems unlikely. If the responsivity changed during the measurement, we would observe a proportional loss in power at all of the currents levels. We, however, observe an increase in power that is not proportional because the percent increase depends on the power level. Either the responsivity changed before the measurements started in November 2006 or the calibration has too much uncertainty to be valuable. Part of the problem may be that they perform the calibration at $514 \mathrm{~nm}$ instead of using wavelengths in the infrared. This calibration from Coherent occurred after cycle number 315,855. During this time, the laser sat dormant for a few weeks and no apparent change was observed after the calibration once we corrected for the change in the value of the responsivity programmed into the hardware of the power meter by Coherent. Thus, the data shown in this report uses the initial responsivity of $0.1481 \mathrm{~V} / \mathrm{W}$ for the power meter for all the values provided. 


\subsection{Beam Pointing Stability}

We also investigated the beam pointing stability by measuring the far-field divergence of a QCL along its fast and slow axes. The apparatus used for these measurements is comprised of a rotary stage with a step resolution of 0.5 degrees and a fixed liquid-nitrogen cooled HgCdTe detector with a detection area of 250 microns that is located about $150 \mathrm{~mm}$ from the front facet of the QCL (Krishnaswami et al. 2008). The QCL under test is mounted on a precision block so the emission facet is always located within $0.5 \mathrm{~mm}$ of the axis of rotation, with either the fast or slow axis of the QCL parallel to the rotation axis. We measured the beam pointing stability of a Maxion device, M532A, which is a Fabry-Perot multimode QCL with an HR-coated rear facet that lases around 9.66 $\mu \mathrm{m}$. The laser is AuSn-soldered episide down onto an AlN submount and then $\mathrm{Pb} / \mathrm{Sn}$ soldered onto a C-mount. These tests were performed to verify that varying the current did not induce any angular steering of the output because the current is changed to adjust the output power. No changes in beam shape or pointing were observed when either the duty cycle or the current was changed. Figures 34 and 35 show the beam profile along the fast and slow axes, respectively, as a function of several different current levels.

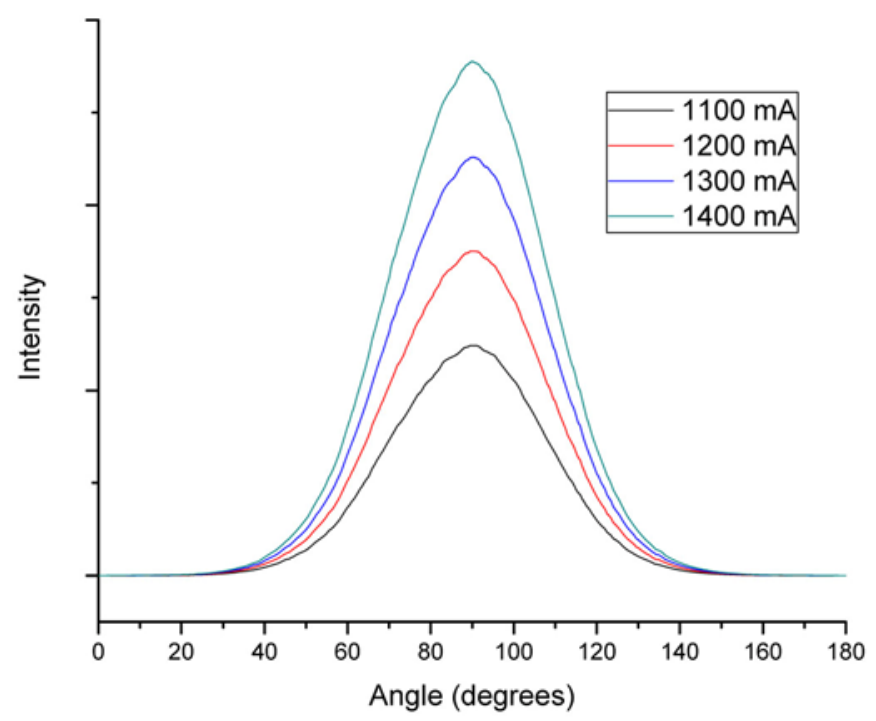

Figure 4.1. Beam Profiles Along the Fast Axis for M532A at Different Current Levels 


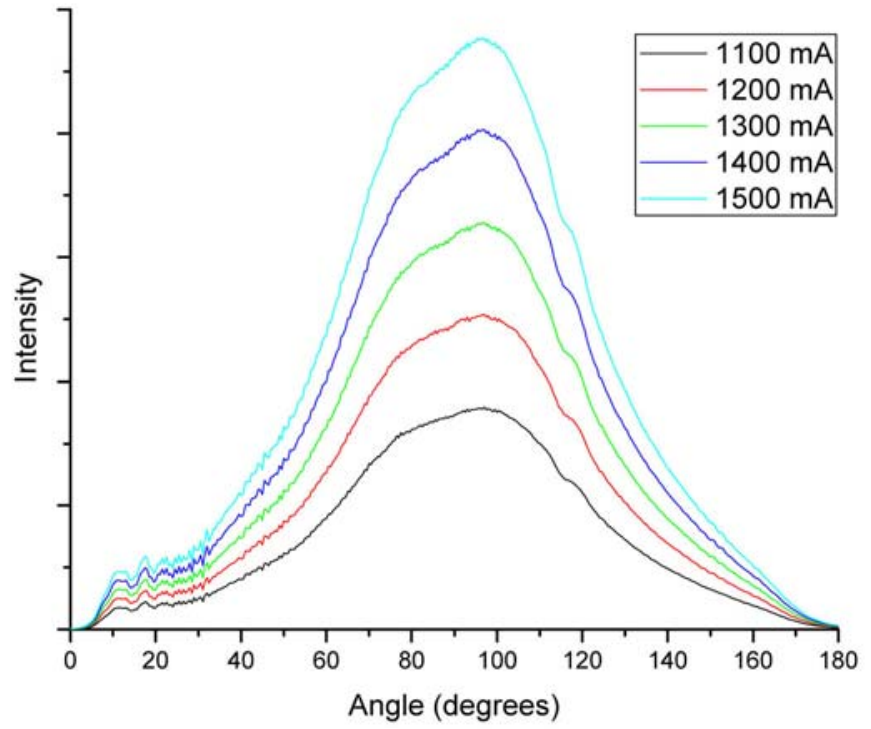

Figure 4.2. Beam Profiles Along the Slow Axis for M532A at Different Current Levels 


\subsection{Summary}

The thermoelectrically cooled QCLs offer a promising alternative to blackbodies. They are robust with power cycling and provide a stable radiometric output. Multiple radiance levels are easily achieved by changing the current rapidly and can be accomplished on a timescale faster than 60 seconds, which is primarily limited by the timescale of the detector. Unlike blackbodies, stability does not require continuous power to the QCLs. Instead, the QCLs can be maintained in stand-by mode when a calibration is not being performed, thereby alleviating the overall power burden of the instrument. Finally, lifetimes that extend out to five years or beyond should be achievable for QCL devices with a proper die attach and high-quality laser material.

The biggest technical challenge with this project has been to measure the QCL stability. In our measurements on the uncollimated Argos QCL, we identified several types of power variation, all but two of which are due to the measurement setup. For the initial measurement setup, changes in the laboratory temperature drove changes in the power meter head zero readings and the current delivered by the current controller. Improved temperature control of these two components reduced the zero-subtracted power reading variations due to laboratory temperature variations to below what we can observe. The manufacturer's specifications for the temperature dependence of the digital-to-analog converter on the NI-6221 data acquisition board are near the limit we would be able to measure and would be significant for further improvements of the measurement setup. A major barrier to such an improvement is the quantization of the output of the 3 Sigma ${ }^{\mathrm{TM}}$ power meter readout that appears to be the source of the noise floor for this setup. Changes in the measurement setup usually resulted in small step-function changes in zero-subtracted power readings and the QCL compliance voltage, even changes that in principle should not have changed the current through the QCL. Many of these step-function changes are consistent with a simple model that assumes changes in contact potentials and current changes smaller than $0.1 \mathrm{~mA}$. This behavior suggests that an integrated stand-alone system should provide even higher stability than the 0.023\% over 16 days that we observed with the uncollimated Argos QCL.

We identified two sources of power variation that appear to be intrinsic to the QCL itself rather than the operating or power measurement conditions. The first is the stochastic variation in the distribution of initial lasing modes that was discovered at the end of FY07, which can be solved by using a highfrequency modulation of the current through the QCL. The second source is the burn-in effect that we have seen in both the cryogenic QCL and the pulsed room-temperature QCL that we have also studied. The Argos QCL showed a small burn-in effect that corresponded to power increases of about $0.1 \mathrm{~mW}$ at all current levels and appeared to be over after about 80 hours of current flowing through this QCL. In contrast, the four continuous-wave room-temperature Fabry-Perot QCLs from Maxion that we studied showed power increases of about $0.5 \mathrm{~mW}$ that were still changing after hundreds of hours of current flowing through the QCLs. Attempts to reduce the magnitude and/or speed up completion of burn-in by thermal annealing and flowing sub-threshold currents at an elevated temperature were not successful. Burn-in is the major limit on power stability for all the Maxion Fabry-Perot QCLs we have studied. Its impact, however, can be minimized by operating the QCL continually above threshold for a few weeks prior to use.

We also looked at the power stability of the Argos QCL after it was re-mounted on the HHL optics platform and collimated. When collimated, this QCL showed stability of $0.06 \%$ over almost 15 days in a 
new setup. Part of this residual variation appears to be because of larger current variations from the current controller used in this new setup. After correcting for this effect, the stability of the collimated Argos QCL was within a factor of 2 of the uncollimated QCL. The source of this additional variability may be due to feedback from the lens into the QCL, but other possibilities include poorer temperature control due to the larger thermal mass of the HHL optics platform. The fact that re-mounting the Argos QCL did not re-start a burn-in process argues that thermal contact between the C-mount and the heat sink is not a factor in the burn-in for this QCL. It is also reassuring that when we assembled a second continuous-wave room-temperature test setup using what we learned from the first setup, we were able to achieve similarly low measurement noise. This confirms that we have a good understanding of the major sources of measurement variation. 


\subsection{References}

Anheier NC, BE Bernacki, BK Hatchell, N Ho, K Krishnaswami and A Qiao. 2008. FY 2007 Infrared Photonics Final Report. PNNL-17392. Pacific Northwest National Laboratory, Richland, Washington.

Fluke. 2008. Thermistor Standards Probes Models 5640-5644. Hart Scientific. American Fork, Utah. Accessed 1/7/2009 at http://www.hartscientific.com/products/5640.htm (last updated 12/31/2008).

Krishnaswami K, BE Bernacki, BD Cannon, N Ho and NC Anheier, Jr. 2008. "Emission and Propagation Properties of Mid-Infrared Quantum Cascade Lasers`.” IEEE Photon. Tech. Lett. 20(4):306308.

Lawton KM and SR Patterson. 2001. "Long-Term Relative Stability of Thermistors.” Precis. Eng. 25(1):24-28.

Liu X, RW Davis, LC Hughes, MH Rasmussen, R Bhat, CE Zah and J Straadling. 2006. "A Study on the Reliability of Indium Solder Die Bonding of High Power Semiconductor Lasers.” J. Appl. Phys. 100:013104.

Lyons P and P Waterworth. 2003. The Use of NTC Thermistors as Sensing Devices for TEC Controllers and Temperature Control Integrated Circuits. Betatherm Ireland Ltd., Galway, Ireland. Available at http://www.betatherm.com/ThermistorTECapplicationnotes.pdf.

Myers TL, BT Broocks, BD Cannon and N Hô. 2007. FY07 Final Report for Calibration Systems. PNNL-17254. Pacific Northwest National Laboratory, Richland, Washington.

Myers TL, BT Broocks, N Hô and MA Phillips. 2006. Calibration Systems Development in FY06. PNNL-16296. Pacific Northwest National Laboratory, Richland, Washington.

Wise JA. 1992. Stability of Glass-Encapsulated Disc-type Thermistors. National Institute of Standards and Technology, Gaithersburg, Maryland. Available at http://www.cstl.nist.gov/div836/836.05/papers/Wise92glass-disc thermistors.pdf. 

Appendix A

Thermopile Amplifier 



\section{Appendix A}

\section{Thermopile Amplifier}

The 3Sigma ${ }^{\mathrm{TM}}$ power meter readout amplifies and digitizes the voltage output from the power meter head, but the $0.01 \mathrm{~mW}$ resolution of the output of 3 Sigma $^{\mathrm{TM}}$ dominates the short-term noise of the power readings. Figure A.1 shows the schematic of the amplifier whose input was connected to the output of the power meter head in parallel with the connection to the 3 Sigma $^{\mathrm{TM}}$. This circuit came from the data sheet $^{(\mathrm{a})}$ for the LTC1047 dual zero-drift operational amplifier and was modified by changing the value of R9 to change the gain from 10,000 to 1000 and replacing the unavailable JFET transistors with available low-noise versions. Two versions of this amplifier were built and tested, one with a pair of discrete lower noise JFETs (Model 2SK369) and the other with a dual JFET (Model LSK389). The dual JFET has both JFETs in the same package and on the same silicon substrate for much better temperature matching and matching of device characteristics. The amplifier with the dual JFET gave the best performance and will be described further.

The pair of JFETs, J1a and J1b, is the very low-noise input stage of this amplifier. The operational amplifier U2 provides additional gain and the output is fed back via the voltage divider made of R8 and $\mathrm{R} 9$ to the gate of J1b, which is the inverting input of this amplifier. These resistors are ultra-low temperature coefficient to maintain a constant gain. The operational amplifier U1 integrates the voltage difference between the gates of J1a and J1b on capacitor C1, and U1's output adjusts the voltage on the source of J1a to drive this voltage difference to zero, which cancels out drifts in this amplifier. U1 and U2 are low-noise zero-drift chopper-stabilized operational amplifiers that are able to cancel long-term drifts. This amplifier was built into a DB25 pass-through connector shown in Figure A.2 with the connections shown in Figure A.3.

A major shortcoming of this amplifier design, which was not mentioned in the data sheet or other application notes on this design, is the very poor rejection of low-frequency noise on the power supply rails, especially the positive rail. Unfortunately, this poor power supply rejection meant that the $\pm 5 \mathrm{~V}$ supplies from the 3 Sigma ${ }^{\mathrm{TM}}$ could not be used to power this amplifier. Building a separate power supply using a low-noise voltage reference (model REF02) and a pair of operational amplifiers solved this problem, but at the cost of added complexity and additional potential ground loops.

With the input shorted with a 100-ohm resistor, the standard deviation of the output noise over 20 minutes at 25 samples per second was $80-90 \mu \mathrm{V}$ and $1.2 \mathrm{mV}$ peak-to-peak. This is significantly larger than the $95 \mu \mathrm{V}$ peak-to-peak over 10 seconds in a $10-\mathrm{Hz}$ bandwidth given in the datasheet, but the measurement time is 120 times longer. However, when connected to a power meter head out in the lab in parallel to a 3 Sigma $^{\mathrm{TM}}$ readout, a similar 20-minute measurement gave an output standard deviation of $800 \mu \mathrm{V}$, which corresponds to a standard deviation in the power reading from the thermopile head of $0.005 \mathrm{~mW}$, which is reasonable for this setup. Traces c) and d) in Figure 2.11 show that with the head in the custom environmental chamber, this amplifier gives much lower readout noise than the 3 Sigma ${ }^{\mathrm{TM}}$ over a 10-day period. Unfortunately, it also appears to have caused a slow power drift.

(a) http://www.linear.com/pc/downloadDocument.do?navId=H0,C1,C1154,C1009,C1021,P1685,D2842 


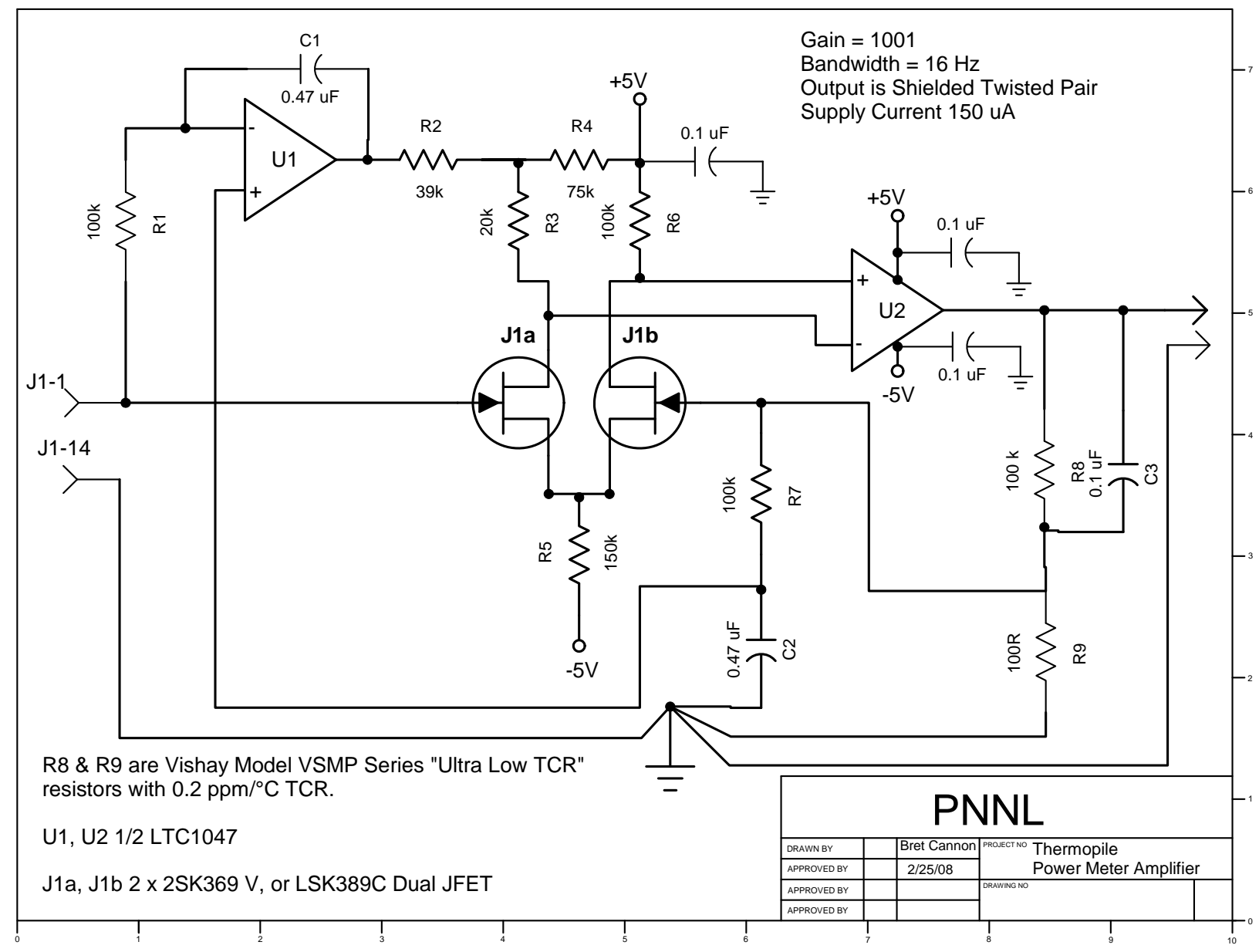

Figure A.1. Schematic of Low-Noise Amplifier Used in Parallel with 3Sigma ${ }^{\mathrm{TM}}$ Power Meter Readout

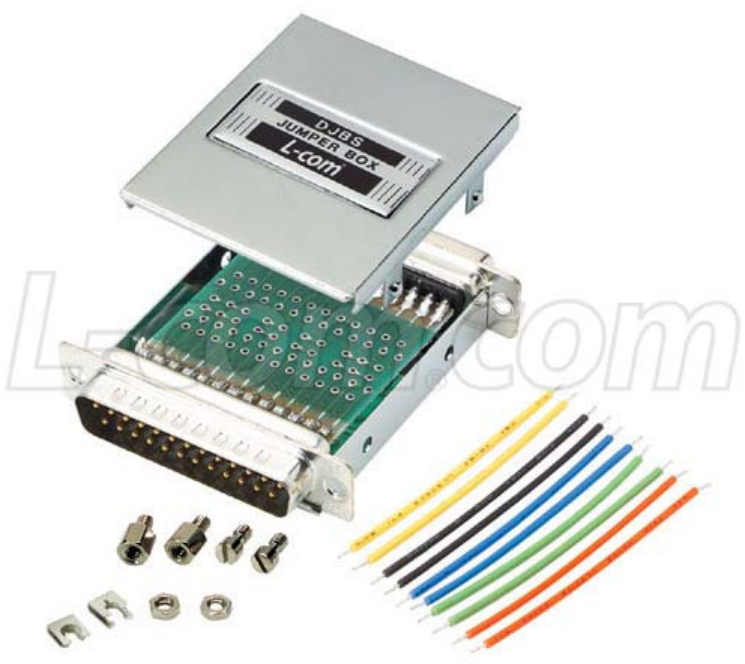

Figure A.2. DB25 Pass-Through Connector Used to House Amplifier in Figure A.1 


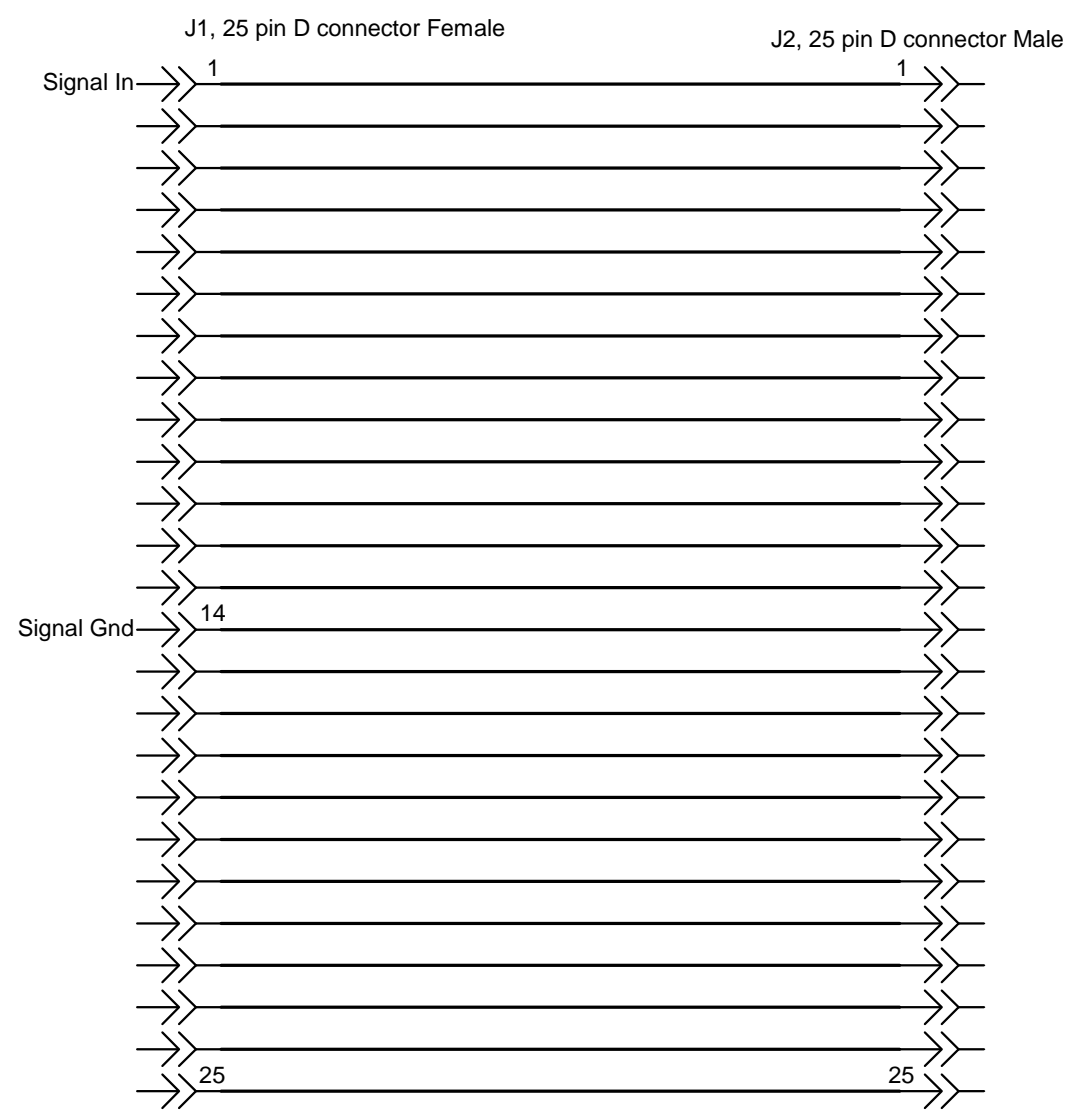

Figure A.3. Connection Diagram for Amplifier in Figure A.1 to the DB25 Pass-Through Connector Shown in Figure A.2. 



\section{Distribution}

No. of

Copies

\# Name

Organization

Address

City, State and Zip Code

\# Name

Organization

Address

Address line 2

COUNTRY

\# Organization

Address

City, State and Zip Code

Name

Name

Name

Name

Name (\#)
No. of

Copies

\# Name

Organization

Address

City, State and Zip Code

\# Internal Distribution

Pacific Northwest National Laboratory

P.O. Box 999

Richland, WA 99352

Name

Mailstop

Name

Mailstop

Name

Mailstop

Name

Mailstop

Name

(PDF) 


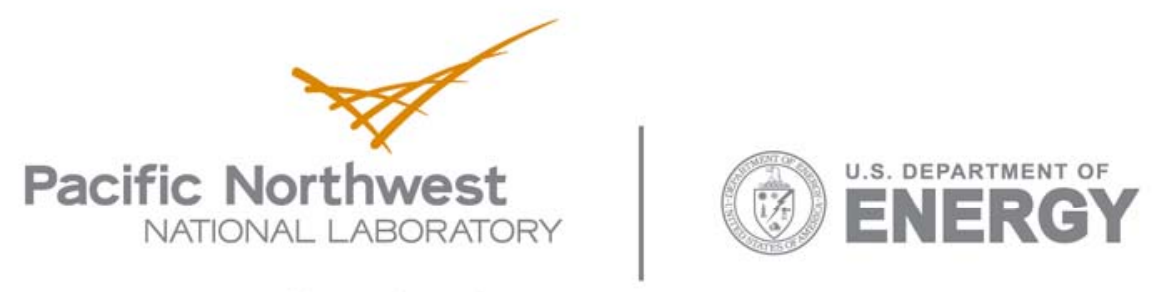

902 Battelle Boulevard

P.O. Box 999

Richland, WA 99352

1-888-375-PNNL (7665)

www.pnl.gov 